\title{
RELATIVE MOTIVES AND THE THEORY OF PSEUDO-FINITE FIELDS
}

\author{
JOHANNES NICAISE
}

\begin{abstract}
We generalize the motivic incarnation morphism from the theory of arithmetic integration to the relative case, where we work over a base variety $S$ over a field $k$ of characteristic zero. We develop a theory of constructible effective Chow motives over $S$, and we show how to associate a motive to any $S$-variety. We give a geometric proof of relative quantifier elimination for pseudo-finite fields, and we construct a morphism from the Grothendieck ring of the theory of pseudo-finite fields over $S$, to the tensor product of $\mathbb{Q}$ with the Grothendieck ring of constructible effective Chow motives. This morphism yields a motivic realization of parameterized arithmetic integrals. Finally, we define relative arc and jet spaces, and the three relative motivic Poincaré series.
\end{abstract}

\section{INTRODUCTION}

Let $k$ be a field of characteristic zero. Consider the Grothendieck ring $K_{0}\left(P F F_{k}\right)$ of the theory of pseudo-finite fields over $k$ (Section 2.2), and denote by $K_{0}^{m o t}\left(\operatorname{Var}_{k}\right.$ ) the image of the Grothendieck ring of $k$-varieties in the Grothendieck ring of Chow motives, under the well-known morphism $\chi_{c}: K_{0}\left(\operatorname{Var}_{k}\right) \rightarrow K_{0}\left(\operatorname{CMot}_{k}\right)$, see [22], [27, or [5] for a short construction using weak factorization. Denef and Loeser [13] constructed a generalized motivic Euler characteristic

$$
\chi_{(c)}: K_{0}\left(P F F_{k}\right) \rightarrow K_{0}^{m o t}\left(\operatorname{Var}_{k}\right) \otimes \mathbb{Q}
$$

For this construction, it is important to understand the structure of $K_{0}\left(P F F_{k}\right)$. The theory of quantifier elimination for pseudo-finite fields [19] 20] states that quantifiers can be eliminated if one adds some relations to the language of rings, which have a geometric interpretation in terms of Galois covers (Section 3). This interpretation yields a construction for $\chi_{(c)}$. The morphism $\chi_{(c)}$ provides a concrete motivic realization of the theory of arithmetic motivic integration [13].

The goal of the present article, is to generalize this construction to the relative case, where we work over an arbitrary $k$-variety $S$ instead of over the field $k$, in order to obtain a motivic incarnation of arithmetic integrals with parameters, part of a work in progress by Cluckers and Loeser [7, 8, 9].

In Sections 2-4, we study the theory of pseudo-finite fields (Definition 2.1) over the base variety $S$. We define the relative Grothendieck ring $K_{0}\left(P F F_{S}\right)$ in Section 2.2 and we give a purely geometric proof of quantifier elimination for ring formulas over $S$ w.r.t. the theory of pseudo-finite fields, in terms of Galois formulas.

We briefly illustrate the concept of quantifier elimination by means of a classic example. Let $\mathcal{L}$ be the first order language consisting of two binary function symbols, a binary relation symbol, and two constant symbols, to denote,.,$+<, 0$ and 1 , respectively. These symbols are interpreted in a structure with universe $\mathbb{R}$ in the obvious way. By abuse of notation, we denote this structure again by $\mathbb{R}$. 
Tarski proved that $\mathbb{R}$ allows elimination of quantifiers, meaning that there exists, for each formula $\psi\left(v_{1}, \ldots, v_{m}\right)$ in $\mathcal{L}$, a formula $\varphi\left(v_{1}, \ldots, v_{m}\right)$ in $\mathcal{L}$, without quantifiers, such that the set of $m$-tuples $\left(x_{1}, \ldots, x_{m}\right) \in \mathbb{R}^{m}$ for which $\psi\left(x_{1}, \ldots, x_{m}\right)$ holds in $\mathbb{R}$, is exactly the same as the set of $m$-tuples making the formula $\varphi$ true in $\mathbb{R}$. In other words: every definable subset of $\mathbb{R}^{m}$ can be described by a quantifier-free formula. This is a very important property: it helps us to understand the structure of definable sets, since quantifier-free formulas are (in general) more transparent than formulas with quantifiers. The key is to find a language describing a particular structure that is sufficiently rich to allow elimination of quantifiers, but sufficiently simple to keep quantifier-free definable sets transparent.

Fried and Jarden developed in 19] a quantifier elimination procedure for the theory of pseudo-finite fields by introducing so-called Galois formulas (defined in Section 3). Theorem 4.1 gives a purely geometric proof of this result in the relative setting. It states that any ring formula over $S$ is equivalent to a quantifier-free Galois formula, where equivalent means that they define the same sets when interpreted over an $M$-valued point of $S$, with $M$ a pseudo-finite field.

To define a relative analogue of the Grothendieck ring $K_{0}^{m o t}\left(\operatorname{Var}_{k}\right)$, we need an appropriate theory of motives over an arbitrary base variety $S$ over $k$. The category $C$ Mot $_{S}$ of constructible effective Chow motives over $S$ is constructed in Section 6.8 as a direct limit of

$$
\prod_{S_{i} \in \mathscr{S}} \mathcal{M}_{+}^{o}\left(S_{i}\right)
$$

where $\mathscr{S}$ runs over the finite stratifications of $S$ into smooth, irreducible locally closed subsets, and $\mathcal{M}_{+}^{o}\left(S_{i}\right)$ is the category of effective Chow motives over $S_{i}$ defined in 17 .

For any finite group $G$, we construct a functor from the category $\left(G, \operatorname{Var}_{S, c}\right)$ of $S$-varieties with good $G$-action (with proper morphisms), to the homotopy category $H o\left(G, \mathcal{C}^{b}\left(\mathrm{CMot}_{S}\right)\right)$ of the additive category $\left(G, C M o t_{S}\right)$ of constructible motives with $G$-action. The construction is based on a generalization of the extension principle for cohomological functors 27, using the existence of constructible resolution of singularities over $S$ (Proposition 5.4) and the existence of a split exact blowup sequence for relative effective Chow motives (Theorem 6.12). In particular, we obtain a morphism of Grothendieck rings

$$
\chi_{c}: K_{0}\left(\operatorname{Var}_{S}\right) \rightarrow K_{0}\left(\operatorname{CMot}_{S}\right)
$$

whose image we denote by $K_{0}^{m o t}\left(\operatorname{Var}_{S}\right)$.

We define induction and restriction functors on $H o\left(G, \mathcal{C}^{b}\left(C M o t_{S}\right)\right)$ with respect to morphisms of finite groups, and we prove their main properties. The most important feature of this formalism is the fact that the motive of a quotient variety $X / G$ coincides with the $G$-invariant part of the motive of $X$ (Theorem 7.8 ). Using the induction and restriction functors, we define a character decomposition of the motive of an object in $\left(G, \operatorname{Var}_{S}\right)$. This decomposition satisfies certain Frobenius reciprocity properties (Lemma 7.18] and Lemma 7.20).

Now we are ready to construct the motivic realization morphism

$$
\chi_{(c)}: K_{0}\left(P F F_{S}\right) \rightarrow K_{0}^{m o t}\left(\operatorname{Var}_{S}\right) \otimes \mathbb{Q}
$$

Quantifier elimination over pseudo-finite fields, and the geometric nature of Galois formulas, suggest a natural definition of $\chi_{(c)}$. The subtle part is the proof that 
equivalent formulas (i.e. formulas for which there exists a definable bijection between the sets they define over pseudo-finite fields) define the same motive; this follows from the Frobenius reciprocity properties of the character decomposition.

Finally, we introduce relative arc and jet spaces. If $X$ is a separated scheme of finite type over $S$, the $n$-th jet scheme $\mathcal{L}_{n}(X / S)$ is a separated $S$-scheme of finite type that parametrizes $\left(S \times_{k} k[t] /\left(t^{n+1}\right)\right)$-valued sections on $X$. The arc scheme $\mathcal{L}(X / S)$ is defined as a projective limit of the jet schemes, and is endowed with natural projection morphisms

$$
\pi_{n}: \mathcal{L}(X / S) \rightarrow \mathcal{L}_{n}(X / S)
$$

We define the relative motivic Igusa Poincaré series (measuring the jet schemes $\mathcal{L}_{n}(X / S)$ ), as well as the relative geometric Poincaré series (measuring the projections $\pi_{n} \mathcal{L}(X / S)$ ) and the arithmetic Poincaré series (measuring the projections $\pi_{n}(\mathcal{L}(X / S)(K))$ for pseudo-finite fields $\left.K\right)$. To show that the latter two are welldefined, we establish a uniform version of Greenberg's Theorem [23, based on a short new proof of the absolute case. This result guarantees that the projections in the definition are measurable (i.e. constructible, resp. definable by a ring formula).

Let us give an overview of the results in this paper. In Section 2] we formulate some basic results concerning Galois covers and pseudo-finite fields, and we introduce the relative Grothendieck ring of the theory of pseudo-finite fields. In Section 3. we define relative Galois stratifications, as well as some basic operations on these stratifications. Section 4 is devoted to a geometric proof of the elimination theorem (Theorem 4.11).

In Section 5 we prove the existence of constructible equivariant resolution of singularities for varieties over $S$ with an action of a finite group, and a constructible equivariant Hironaka-Chow Lemma. Section [6] is devoted to the theory of constructible motives over $S$. Sections 6.3 to 6.6 contain some important properties of relative effective Chow motives over a smooth base, in particular the existence of a split exact blow-up sequence (Theorem 6.12). In Section 6.8 we define our category of constructible motives over $S$. The generalized extension criterion for cohomological functors is stated in Section 6.9 and applied in Section 6.10 to associate a constructible motive with $G$-action to any $S$-variety $X$ with good $G$-action.

We define a character decomposition for constructible motives in Section 7 and we prove its main properties. A crucial result in this context is the fact that the motive of a quotient $X / G$ coincides with the $G$-invariant part of the motive of $X$ (Theorem [7.8). In Section 8] Theorem 8.7 we construct a motivic incarnation morphism

$$
\chi_{(c)}: K_{0}\left(P F F_{S}\right) \rightarrow K_{0}^{m o t}\left(\operatorname{Var}_{S}\right) \otimes \mathbb{Q}
$$

for the relative theory of pseudo-finite fields.

Finally, in Section 9] we introduce relative arc and jet spaces, and we prove a uniform version of Greenberg's Theorem, which allows us to define the three relative motivic Poincaré series.

This paper borrows basic notions and techniques from first order logic and model theory. A nice introduction to these fields is given in 32. In the absolute case $S=\operatorname{Spec} k$, we recover the construction by Denef and Loeser [13. In our proofs, however, we avoided any passage to finite fields, and hence any reference to ultraproducts, merely using the geometric-arithmetic properties of pseudo-finite fields 
established in Section 2 Our proof of the quantifier elimination property in Theorem 4.1 relies entirely on the existence of a certain short exact sequence of algebraic fundamental groups. We hope this makes the presentation more accessible to nonmodel theorists.

Throughout the paper, $k$ denotes a field of characteristic zero. A variety over a scheme $S$ is a separated reduced scheme, of finite type over $S$. For any scheme $S$, we denote by $S_{\text {red }}$ the underlying reduced scheme.

\section{PSEUdo-FINITE FIELDS}

\subsection{Pseudo-finite fields and Galois covers.}

Definition 2.1. A pseudo-algebraically closed field (PAC-field) is a field $M$, such that every geometrically irreducible $M$-variety has an $M$-rational point.

A pseudo-finite field $M$ is an infinite perfect PAC-field, which has exactly one field extension of degree $n$, for every integer $n>0$, in a fixed algebraic closure of $M$.

Every field can be embedded in a pseudo-finite field [19 23.1.2]. An algebraic extension of a PAC field is PAC, by [19, 11.2.5]. As a consequence, a finite extension of a pseudo-finite field is pseudo-finite. Every finite extension of a pseudo-finite field is cyclic. Ax $[3$ proved that two ring formulas over $\mathbb{Q}$ are equivalent when interpreted in $\mathbb{F}_{p}$, for all sufficiently large primes $p$, if and only if they are equivalent when interpreted in $K$, for all pseudo-finite fields $K$ containing $\mathbb{Q}^{1}$. Hence, pseudo-finite fields allow you to solve certain problems concerning finite fields in characteristic zero.

Definition 2.2 (Galois cover). A Galois cover $h: Y \rightarrow X$ (also denoted by $Y / X$ ) is an étale morphism of separated, integral, normal schemes, satisfying the following property: there exists a finite group $G$, acting faithfully on $Y$, and $h: Y \rightarrow X$ is a quotient map for this action [1, V.1].

The group $G$ (the group of $X$-automorphisms of $Y$ ) is called the Galois group of the Galois cover $Y / X$, and is denoted by $G(Y / X)$.

Definition 2.3 (Decomposition group). Let $h: Y \rightarrow X$ be a Galois cover, and let $Z \rightarrow X$ be a morphism of separated, integral, normal schemes. Let $Z^{\prime}$ be a connected component of $Y \times_{X} Z$. The Galois group $G(Y / X)$ acts on $Y \times_{X} Z$. The decomposition group $D_{Y / X}\left(Z^{\prime} / Z\right)$ of $Z$ at $Z^{\prime}$ with respect to $Y / X$, is the subgroup of elements of $G(Y / X)$ which map the component $Z^{\prime}$ to itself. It coincides with the decomposition subgroup $D_{Y / X}\left(\eta_{Z^{\prime}} / \eta_{Z}\right)$, where $\eta_{Z^{\prime}}$ and $\eta_{Z}$ are the generic points of $Z^{\prime}$, resp. $Z$.

The morphism $Z^{\prime} \rightarrow Z$ is a Galois cover, and the decomposition group of $Z$ at $Z^{\prime}$ is canonically isomorphic to the Galois group $G\left(Z^{\prime} / Z\right)=G\left(\eta_{Z^{\prime}} / \eta_{Z}\right)$. As $Z^{\prime}$ runs through the connected components of $Y \times_{X} Z$, the decomposition group of $Z$ at $Z^{\prime}$ runs through its conjugation class in $G(Y / X)$. We call this conjugation class the decomposition class of $Z$ (w.r.t. $Y / X)$, and denote it by $C_{Y / X}(Z)$.

We list three easy properties of the decomposition group for later use.

\footnotetext{
${ }^{1} \mathrm{~A}$ ring formula over a field $k$ is a logical formula $\varphi$ built from Boolean combinations of polynomial equalities over $k$, and quantifiers.
} 
Lemma 2.4. Let $Y / X$ be a Galois cover, let $M$ be a field, and let $x$ be an $M$ valued point on $X$. Let $x^{\prime}$ be a connected component of $Y \times_{X} x$. Then $D_{Y / X}\left(x^{\prime} / x\right)$ is the smallest subgroup $H$ of $G(Y / X)$, such that the point $x \rightarrow X$ lifts to a point $x \rightarrow Y / H$, making the diagram

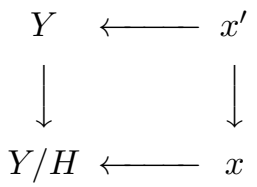

commute.

Lemma 2.5. Let $Y / X$ be Galois cover, let $V$ be a separated, normal, integral scheme over $X$, and let $W$ be any connected component of $V \times_{X} Y$. Let $A$ be a separated, normal, integral scheme over $V$, and let $B$ be any connected component of $A \times_{V} W$. If we denote by $B^{\prime}$ the image of $B$ in $A \times_{X} Y$, then $D_{Y / X}\left(B^{\prime} / A\right)=$ $D_{W / V}(B / A)$ (where we view both sides as subgroups of $\left.G(Y / X)\right)$.

Lemma 2.6. Let $Z / X$ be Galois cover, let $H$ be a normal subgroup of the Galois group $G(Z / X)$, and let $Y$ be the quotient $Z / H$. Let $V$ be a separated, integral, normal scheme over $X$, let $V_{Z}$ be any connected component of $Z \times_{X} V$, and let $V_{Y}$ be its image in $Y \times_{X} V$. Then $D_{Y / X}\left(V_{Y} / V\right)$ is the image of $D_{Z / X}\left(V_{Z} / V\right)$ under the restriction map $G(Z / X) \rightarrow G(Y / X)$.

A valuable property of pseudo-finite fields is given in the following lemma; see [19. 24.1.4] (pseudo-finite fields are Frobenius fields).

Lemma 2.7. Let $Y \rightarrow X$ be a Galois cover, where $X$ and $Y$ are varieties over a pseudo-finite field $M$ of characteristic zero, and $X$ is geometrically irreducible over $M$. Denote by $N$ be the algebraic closure of $M$ in the function field $k(Y)$. If $H$ is a cyclic subgroup of the Galois group $G(Y / X)$, such that the image of $H$ under the restriction morphism $G(Y / X) \rightarrow G(N / M)$ is the whole Galois group $G(N / M)$, then there exist an $M$-rational point $x$ on $X$ such that $H$ belongs to $C_{Y / X}(x)$.

This lemma can be seen as an "optimal lifting property": for each reasonable candidate, you find a rational point $x$ on $X$ with the desired decomposition group.

Lemma 2.8. Let $Y \rightarrow X$ a Galois cover, with $X$ and $Y$ varieties over $k$. If $C=\langle\sigma\rangle$ is a cyclic subgroup of the Galois group $G(Y / X)$, then there exists a closed $M$-valued point $x$ on $X$, with $M$ a pseudo-finite field, such that $C$ belongs to $C_{Y / X}(x)$. Moreover, these points are dense in $X$.

Proof. If $U$ is a non-empty open subscheme of $X$, then $Y \times_{X} U / U$ is a new Galois cover with the same Galois group. Hence, if there exist one such $x$, these points are dense in $X$.

Let $k^{\prime}$ be the algebraic closure of $k$ in the function field $k(X)$ of $X$. There exists a nonempty open subset $U$ of $X$, such that $U$ is defined over $k^{\prime}$. Hence, we may assume that $k=k^{\prime}$.

Let $K$ be the algebraic closure of $k$ in the function field $k(Y)$, let $\sigma^{\prime}$ be the image of $\sigma$ in $G(K / k)$, and denote by $K^{\sigma^{\prime}}$ its fixed field. Since $G\left(K / K^{\sigma^{\prime}}\right)$ is cyclic, there exists a pseudo-finite extension $M$ of $K^{\sigma^{\prime}}$, which is linearly disjoint from $K$ over $K^{\sigma^{\prime}}$, by [19, 23.1.1].

The embedding of $K^{\sigma^{\prime}}$ in $K$ defines a connected component $Y^{\prime}$ of $Y \times_{k} K^{\sigma^{\prime}}$. The cover $Y^{\prime} / X \times_{k} K^{\sigma^{\prime}}$ is Galois, and its Galois group is the decomposition group 
of $X \times_{k} K^{\sigma^{\prime}}$ at $Y^{\prime}$ w.r.t. $Y / X$. It contains the element $\sigma$. Since $M$ is linearly disjoint from $K$ over $K^{\sigma^{\prime}}$, the space $Y^{\prime} \times_{K^{\sigma^{\prime}}} M$ is connected, and the Galois group $G\left(Y^{\prime} \times{ }_{K^{\sigma^{\prime}}} M / X \times_{k} M\right)$ is isomorphic to $G\left(Y^{\prime} / X \times_{k} K^{\sigma^{\prime}}\right)$. In particular, it contains $\sigma$ (if we identify $G\left(Y^{\prime} \times{ }_{K^{\sigma^{\prime}}} M / X \times_{k} M\right.$ ) with its image in $G(Y / X)$ ).

By Lemma 2.7 we can find a $M$-rational point $x$ on $X \times_{k} M$, whose decomposition class w.r.t. $Y^{\prime} \times_{K^{\sigma^{\prime}}} M / X \times_{k} M$ contains $C$. By Lemma 2.5 its decomposition class w.r.t. $Y / X$ contains $C$, as well.

Remark. Lemma 2.8 can be seen as a (weak) pseudo-finite version of ArtinChebotarev's Density Theorem [35]: assume that $X, Y$ are irreducible schemes of finite type over $\mathbb{Z}$, of dimension $\geq 1$, and that $Y / G=X$ for some finite group $G$ acting on $Y$, such that $G$ operates freely on $Y$, and faithfully on the residue field of the generic point of $Y$. If $C$ is any subset of $G$, stable under conjugation, then the set of closed points $x$ of $X$ for which $F_{x} \in C$, has Dirichlet density equal to $|C| /|G|$. Here $F_{x}$ is the Frobenius element in $G(k(y) / k(x))$, where $y$ is some closed point of $Y$, lying over $x$.

2.2. The Grothendieck ring of the theory of pseudo-finite fields. Let $S=$ Spec $R$ be an affine scheme. We consider the relative Grothendieck ring $K_{0}\left(P F F_{R}\right)$ of the theory of pseudo-finite fields over $R$. As an abelian group, it is generated by classes $[\varphi]$, where $\varphi$ is a ring formula over $R$, which are subject to the relations

$$
\left[\varphi_{1} \vee \varphi_{2}\right]=\left[\varphi_{1}\right]+\left[\varphi_{2}\right]-\left[\varphi_{1} \wedge \varphi_{2}\right]
$$

whenever $\varphi_{1}$ and $\varphi_{2}$ have the same free variables. For any pair of ring formulas $\varphi_{1}, \varphi_{2}$ over $R$, with free variables, we impose the additional relation $\left[\varphi_{1}\right]=\left[\varphi_{2}\right]$, whenever there exists a ring formula $\psi$ over $R$ such that, for any pseudo-finite field $M$ and any element $x$ of $S(M)$, the interpretation of $\psi$ over $M$ defines the graph of a bijection between the tuples over $M$ satisfying $\varphi_{1}$, and those satisfying $\varphi_{2}$. Ring formulas over $R$ are interpreted over $M$ in the obvious way, via the ring morphism $R \rightarrow M$ corresponding to $x$. We denote this equivalence relation on ring formulas over $R$ by $\varphi_{1} \equiv_{S} \varphi_{2}$, and we say that $\psi$ defines an equivalence between $\varphi_{1}$ and $\varphi_{2}$ over $S$. If $\varphi$ is a ring formula without free variables, we make $\varphi$ equivalent to $\varphi^{\prime}:=\varphi \wedge(v=0)$, where $v$ is a (free) variable, and we impose $[\varphi]=\left[\varphi^{\prime}\right]$.

Example: Definable bijections. Let $k$ be a field of characteristic zero, and put $R=k[z]$, and $S=\operatorname{Spec} R$. Let $f_{1}\left(x_{1}\right)$ and $f_{2}\left(x_{2}\right)$ be polynomials in one variable over $k$, and consider the ring formulas $\varphi_{1}=" f_{1}\left(x_{1}\right)=z "$ and $\varphi_{2}=" f_{2}\left(x_{2}\right)=z "$ over $R$. Suppose that, for each point $z_{0}$ of $S$, and each field extension $M$ of $k\left(z_{0}\right)$, the number of elements $x_{1}$ that satisfy $\varphi_{1}$ over $M$, equals the number of elements $x_{2}$ that satisfy $\varphi_{2}$ over $M$.

A trivial example is the case where $f_{1}$ and $f_{2}$ are linearly related, i.e. there exist $a, b$ in $R$, with $a$ a unit, such that $f_{2}(x)=f_{1}(a x+b)$. The ring formula $\psi=$ " $x_{1}=a x_{2}+b$ " defines an equivalence between $\varphi_{1}$ and $\varphi_{2}$ over $S$.

On the other hand, suppose $\operatorname{deg}\left(f_{1}\right)=\operatorname{deg}\left(f_{2}\right)=n$. For each integer $m \geq 0$, and for $i=1,2$, there exists a ring formula $\eta_{i, m}$ over $R$, without free variables, such that, for each point $z_{0}$ of $S=\operatorname{Spec} R$, and each field extension $M$ of $k\left(z_{0}\right)$, the formula $\eta_{i, m}$ is true over $M$, iff the equation $f_{i}(x)=z_{0}$ has exactly $m$ distinct solutions over $M$. Hence, we can build a ring formula $\eta$ over $R$, without free variables, such 
that for each point $z_{0}$ of $S$, and any field extension $M$ of $k\left(z_{0}\right)$, the formula $\eta$ is true over $M$, iff the solutions of $f_{1}\left(x_{1}\right)=z_{0}$ over $M$ are in bijective correspondence with the solutions of $f_{2}\left(x_{2}\right)=z_{0}$ over $M$. However, this does not mean that we can define (the graph of) such a bijection by means of a ring formula over $R$. So, even if $\eta$ is true for all $z_{0}$ and all $M$, we cannot conclude that $\varphi_{1} \equiv_{S} \varphi_{2}$.

We emphasize that, in our definition of $\equiv_{S}$, it is important to consider every point of $S$, not only the closed points. For example, let $p$ be a prime number, and let $R$ be the local ring of Spec $\mathbb{Z}$ at $(p)$, with closed point $x$, and generic point $\eta$. Then $(p .1=0) \equiv_{x}(1=1)$, while $(p \cdot 1=0) \not_{\eta}(1=1)$.

However, when $R$ is a ring of finite type over $\mathbb{Z}$, and $\varphi_{1}, \varphi_{2}, \psi$ are ring formulas over $R$, then $\psi$ defines an equivalence of $\varphi_{1}$ and $\varphi_{2}$ over $S$, iff, for each closed point $x$ of $S$, the formula $\psi$ defines an equivalence of $\varphi_{1}$ and $\varphi_{2}$ over $x$. This follows from Ax' characterization of pseudo-finite fields as ultraproducts of finite fields 3 .

In fact, we will show in Section 4 Corollary 4.5 that the same holds when $R$ is a ring of finite type over any field $k$ of characteristic zero, using quantifier elimination.

Ring multiplication in the Grothendieck ring is induced by taking the conjunction of formulas in disjoint sets of free variables: if $\varphi_{1}$ and $\varphi_{2}$ are ring formulas over $R$, with disjoint sets of free variables, then we put $\left[\varphi_{1}\right] \cdot\left[\varphi_{2}\right]:=\left[\varphi_{1} \wedge \varphi_{2}\right]$. This operation is well-defined, and extends bilinearly to a ring product on $K_{0}\left(P F F_{R}\right)$.

In order to understand the ring $K_{0}\left(P F F_{R}\right)$, we need a relative quantifier elimination procedure, which is described in Section 4 .

If $S$ is, more generally, a Noetherian scheme, we define $K_{0}\left(P F F_{S}\right)$ as the ring of global sections of the unique Zariski-sheaf $\mathcal{F}(S)$ of rings on $S$, such that, for each affine open subscheme $U=\operatorname{Spec} V$ of $S$, the ring of sections $\mathcal{F}(S)(U)$ is equal to $K_{0}\left(P F F_{V}\right)$. If $S=\coprod_{i} U_{i}$ is a finite stratification of $S$ into locally closed affine subschemes $U_{i}$, the sheaf $\mathcal{F}(S)$ equals $\prod_{i} j_{!}^{(i)} \mathcal{F}\left(U_{i}\right)$, where $j^{(i)}$ is the inclusion of $U_{i}$ in $S$. This is a consequence of relation (II), since, for each $f$ in $R$, and each ring formula $\varphi$ over $R$, we have $[\varphi]=[\varphi \wedge f=0]+[\varphi \wedge f \neq 0]$.

Similarly, a ring formula $\varphi$ of $S$ consists of a finite stratification $S=\coprod_{i} U_{i}$ of $S$ into locally closed affine subschemes $U_{i}=\operatorname{Spec} R_{i}$, and a ring formula $\varphi_{i}$ over $R_{i}$ for each $i$. A ring formula $\varphi$ over $S$ defines a class $[\varphi]$ in $K_{0}\left(P F F_{S}\right)$. A quantifierfree ring formula $\varphi$ with $m$ free variables defines a constructible subset of $\mathbb{A}_{S}^{m}$. For any ring formula $\varphi$ over $S$, in $m$ free variables, for any point $x$ on $S$, and for any pseudo-finite field extension $M$ of $k(x)$, we will denote by $Z(\varphi, x, M)$, the subset of $M^{m}$ consisting of the tuples satisfying the interpretation of $\varphi$ over $M$.

If $T$ is another Noetherian scheme, endowed with a morphism $f: T \rightarrow S$, it is clear how to pull back a ring formula over $S$ to a ring formula over $T$.

Lemma 2.9. A morphism $f: T \rightarrow S$ induces a pull-back morphism

$$
f^{*}: K_{0}\left(P F F_{S}\right) \rightarrow K_{0}\left(P F F_{T}\right)
$$

Proof. If $\psi, \varphi_{1}, \varphi_{2}$ are ring formulas over $S$, such that $\psi$ defines an equivalence between $\varphi_{1}$ and $\varphi_{2}$, the pull-back of $\psi$ defines an equivalence between the pullbacks of $\varphi_{1}$ and $\varphi_{2}$. 


\section{Galois stratifications}

3.1. Definitions. We start by recalling the concepts of Galois stratification and Galois formula; see [19, §30] and [13, §2].

We fix a field $k$ of characteristic zero, as well as an irreducible $k$-variety $S$, and we will work in the category $\left(\operatorname{Var}_{S}\right)$ of varieties over $S$.

Definition 3.1. Let $X_{S}$ be a variety over $S$. A normal stratification

$$
<X_{S}, C_{i} / A_{i} \mid i \in I>
$$

of $X_{S}$, is a partition of $X_{S}$ into a finite set of integral and normal locally closed $S$-subvarieties $A_{i}$, each equipped with a Galois cover $C_{i} \rightarrow A_{i}$.

$A$ Galois stratification

$$
\mathcal{A}=<X_{S}, C_{i} / A_{i}, \operatorname{Con}\left(A_{i}\right) \mid i \in I>
$$

is a normal stratification $<X_{S}, C_{i} / A_{i} \mid i \in I>$ with, for each $i \in I$, a family Con $\left(A_{i}\right)$ of cyclic subgroups of the Galois group $G\left(C_{i} / A_{i}\right)$, such that Con $\left(A_{i}\right)$ is stable under conjugation. We call Con $\left(A_{i}\right)$ a conjugation domain for the cover $C_{i} / A_{i}$. The support of $\mathcal{A}$ is the union of strata with non-empty conjugation domain.

For each point $x$ of the base scheme $S$, and for each $S$-variety $Z_{S}$, we denote by $Z_{x}$ the fiber of $Z_{S}$ over $x$, endowed with its reduced structure. Let $\mathcal{A}$ be a Galois stratification of $X_{S}$, let $x$ be a point of $S$, let $M$ be a field extension of $k(x)$, and let $a$ be an $M$-valued point of $X_{x}$, belonging to a stratum $A_{i, x}$. We put $\mathcal{A r}\left(C_{i} / A_{i}, x, a\right):=C_{C_{i} / A_{i}}(a)$. We write $\mathcal{A r}(a) \subset \operatorname{Con}(\mathcal{A})$ for $\mathcal{A r}\left(C_{i} / A_{i}, x, a\right) \subset$ $\operatorname{Con}\left(A_{i}\right)$. To these data, we associate a subset $Z$ of $X_{S}(M)$ as follows:

$$
Z(\mathcal{A}, x, M)=\left\{a \in X_{x}(M) \mid \mathcal{A} r(a) \subset \operatorname{Con}(\mathcal{A})\right\}
$$

By Lemma 2.8 the set $Z(\mathcal{A}, x, M)$ is empty for all pseudo-finite field extensions $M$ of $k(x)$, iff the fiber of the support of $\mathcal{A}$ over $x$ is empty.

Let $\mathcal{A}=<\mathbb{A}_{S}^{m+n}, C_{i} / A_{i}, C o n\left(A_{i}\right) \mid i \in I>$ be a Galois stratification of $\mathbb{A}_{S}^{m+n}$, and let $Q_{1}, \ldots, Q_{m}$ be quantifiers. We denote by $\theta$, or by $\theta(\mathbf{Y})$, the formal expression

$$
\left(Q_{1} X_{1}\right) \ldots\left(Q_{m} X_{m}\right)[\mathcal{A} r(\mathbf{X}, \mathbf{Y}) \subset \operatorname{Con}(\mathcal{A})]
$$

where $\mathbf{X}=\left(X_{1}, \ldots, X_{m}\right)$, and $\mathbf{Y}=\left(Y_{1}, \ldots, Y_{n}\right)$. We call $\theta(\mathbf{Y})$ a Galois formula over $S$ in the free variables $\mathbf{Y}$.

To a Galois formula $\theta$, to a point $x$ of $S$, and to a field extension $M$ of $k(x)$, we associate the set

$$
Z(\theta, x, M)=\left\{\mathbf{b}=\left(b_{1}, \ldots, b_{n}\right) \in M^{n} \mid\left(Q_{1} a_{1}\right) \ldots\left(Q_{m} a_{m}\right) \mathcal{A} r(\mathbf{a}, \mathbf{b}) \subset \operatorname{Con}(\mathcal{A})\right\}
$$

where the quantifiers $Q_{1} a_{1}, \ldots, Q_{m} a_{m}$ run over $M$.

3.2. Ring formulas as Galois formulas. It is easy to see how ring formulas over $S$ can be rewritten as Galois formulas. Working locally, we may suppose $S=\operatorname{Spec} R$. Let $\varphi(\mathbf{Y})$ be a formula in the first order language of rings with coefficients in $R$, and in the free variables $\mathbf{Y}$. Writing $\varphi$ in prenex normal form, we obtain a formula

$$
\left(Q_{1} X_{1}\right) \ldots\left(Q_{m} X_{m}\right)\left[\bigvee_{i=1}^{k} \bigwedge_{j=1}^{l} f_{i, j}(\mathbf{X}, \mathbf{Y})=0 \wedge g_{i, j}(\mathbf{X}, \mathbf{Y}) \neq 0\right]
$$

with $f_{i, j}$ and $g_{i, j}$ in $R[\mathbf{X}, \mathbf{Y}]$. The formula between brackets defines a constructible subset $W$ of $\mathbb{A}_{S}^{m+n}$, and we can always find a stratification of $\mathbb{A}_{S}^{m+n}$ into finitely 
many locally closed, integral, normal $S$-subvarieties $A_{i}$, such that each stratum $A_{i}$ is either contained in $W$, or in its complement. For each $i$, we take the Galois cover $C_{i} \rightarrow A_{i}$ to be the identity, and we define $\operatorname{Con}\left(A_{i}\right)$ as the family containing only the trivial group if $A_{i}$ is contained in $W$, and as the empty family otherwise. In this way, we obtain a Galois formula $\theta$, satisfying $Z(\theta, x, M)=Z(\varphi, x, M)$ for each point $x$ of $S$, and each field extension $M$ of $k(x)$.

3.3. Galois formulas as ring formulas. Now, we show how Galois formula $\theta$ can be rewritten as ring formula $\varphi$, such that $Z(\theta, x, M)=Z(\varphi, x, M)$ for each point $x$ of $S$, and each field extension $M$ of $k(x)$. We may suppose that $S=\operatorname{Spec} R$. It is sufficient to construct, for any integral, normal, locally closed subset $A$ of $\mathbb{A}_{S}^{m}$, for any Galois cover $C / A$, and for any cyclic subgroup $H$ of $G(C / A)$, a ring formula $\varphi_{C / A, H}$ with the following property: for any point $x$ of $S$, and any field extension $M$ of $k(x)$, the set $Z\left(\varphi_{C / A, H}, x, M\right)$ is the set of points $a$ in $A_{x}(M)$, such that $H \in C_{C / A}(a)$.

The locally closed subset $A$ can be defined by means of a ring formula $\varphi_{A}$ over $R$ in $m$ free variables. Any étale cover $D$ of $A$ can be defined by means of a ring formula $\varphi_{D}$ over $R$ in $m+i$ free variables, for some integer $i \geq 0$, such that the morphism $C \rightarrow A$ corresponds to projection on the first $m$ coordinates. Now we can use Lemma 2.4 to construct the formula $\varphi_{C / A, H}$.

Hence, using Galois formulas instead of ring formulas does not alter the class of definable sets. The advantage of the new formalism is the property of quantifier elimination, as we will see in Theorem 4.1 .

3.4. Refinement of Galois stratifications. Let $X_{S}$ be an $S$-variety, and let $\mathcal{A}=<X_{S}, C_{i} / A_{i}, \operatorname{Con}\left(A_{i}\right)>$ be a Galois stratification.

Suppose that each $A_{i}$ is stratified into finitely many integral, normal, locally closed subvarieties $A_{i, j}$. We will extend this stratification to a Galois stratification $\mathcal{A}^{\prime}=<X_{S}, C_{i, j} / A_{i, j}, \operatorname{Con}\left(A_{i, j}\right)>$, such that for each point $x$ of $S$, and each field extension $M$ of $k(x)$,

$$
Z(\mathcal{A}, x, M)=Z\left(\mathcal{A}^{\prime}, x, M\right)
$$

Fix a stratum $A_{i, j}$, and let $C_{i, j}$ be a connected component of $C_{i} \times_{A_{i}} A_{i, j}$. The projection $C_{i, j} \rightarrow A_{i, j}$ is a Galois cover, and its Galois group $G\left(C_{i, j} / A_{i, j}\right)$ is the decomposition group of $C_{i} / A_{i}$ at $C_{i, j}$. We choose $\operatorname{Con}\left(A_{i, j}\right)$ to be the conjugation domain consisting of the members of $C o n\left(A_{i}\right)$ which are contained in $G\left(C_{i, j} / A_{i, j}\right)$. It follows from Lemma 2.5 that the resulting Galois cover $\mathcal{A}^{\prime}$ satisfies our requirements. We say that $\mathcal{A}^{\prime}$ is induced from $\mathcal{A}$ by the refinement $\left\{A_{i, j}\right\}$.

3.5. Pulling back Galois stratifications. The preceding refinement procedure is a special case of a more general construction. Let $f: Y \rightarrow X$ be any morphism of irreducible $S$-varieties, and let $\mathcal{A}$ be a Galois stratification of $X$. We pull back $\mathcal{A}$ to a Galois stratification $\mathcal{B}$ on $Y$ as follows: as underlying stratification, we choose any stratification of $Y$ into finitely many integral, normal, locally closed subsets $B_{j}$, which is finer than the inverse image of the stratification of $X$.

Suppose $f$ maps $B_{j}$ into $A_{i}$, and let $D_{j}$ be any connected component of $B_{j} \times{ }_{A_{i}}$ $C_{i}$. The projection $D_{j} \rightarrow B_{j}$ is a Galois cover $D_{j}$ of $B_{j}$, and the Galois group $G\left(D_{j} / B_{j}\right)$ is $D_{C_{i} / A_{i}}\left(D_{j} / B_{j}\right)$. We define the conjugation domain $\operatorname{Con}\left(B_{j}\right)$ as the set of members of $C o n\left(A_{i}\right)$ which are contained in $G\left(D_{j} / B_{j}\right)$.

We say that $\mathcal{B}$ is induced from $\mathcal{A}$ by $f$. This construction is not canonical, but all choices of a stratification correspond to equivalent Galois formulas, i.e. the sets 
$Z(\mathcal{B}, x, M)$ do not depend on any choices: for any point $x$ on $S$, any field extension $M$ of $k(x)$, and any point $a$ of $Y_{x}(M)$, we have

$$
a \in Z(\mathcal{B}, x, M) \text { iff } f(a) \in Z(\mathcal{A}, x, M)
$$

by Lemma 2.5 .

3.6. Inflating a Galois stratification. Let $C / A$ be a Galois cover, and let $C o n(C / A)$ be a conjugation domain for this cover. Suppose that $D / A$ is a Galois cover that dominates $C / A$ (i.e. $D \rightarrow A$ factors through $C \rightarrow A$ ). We define $C o n(D / A)$ as the set of cyclic subgroups of $G(D / A)$, whose image under the projection map $G(D / A) \rightarrow G(C / A)$ belongs to $C o n(C / A)$. This conjugation domain has the following property: for any field $M$, and any $M$-valued point $x$ on $A, C_{D / A}(x) \subset$ $\operatorname{Con}(D / A)$ iff $C_{C / A}(x) \subset \operatorname{Con}(C / A)$, by Lemma 2.6 We say that $C o n(D / A)$ is obtained by inflating the conjugation domain $\operatorname{Con}(C / A)$ to the cover $D / A$.

Let $X$ be an irreducible $S$-variety. We say that a Galois stratification

$$
\mathcal{A}^{\prime}=<X, C_{i}^{\prime} / A_{i}^{\prime}, \operatorname{Con}\left(C_{i}^{\prime} / A_{i}^{\prime}\right) \mid i \in I>
$$

is obtained from a Galois stratification

$$
\mathcal{A}=<X, C_{i} / A_{i}, \operatorname{Con}\left(C_{i} / A_{i}\right) \mid i \in I>
$$

by inflation, if $A_{i}=A_{i}^{\prime}$ for every $i \in I$ (modulo a permutation of the indices), if $C_{i}^{\prime} / A_{i}$ dominates $C_{i} / A_{i}$ for every $i \in I$, and if the conjugation domain $\operatorname{Con}\left(C_{i}^{\prime} / A_{i}\right)$ is obtained from $\operatorname{Con}\left(C_{i} / A_{i}\right)$ by inflation. In this case,

$$
Z(\mathcal{A}, x, M)=Z\left(\mathcal{A}^{\prime}, x, M\right)
$$

for any point $x$ on $S$, and any field extension $M$ of $k(x)$.

3.7. Conjunction and disjunction of Galois stratifications. Let $X$ be a variety over $S$. Consider Galois stratifications

$$
\mathcal{A}=<Y, C_{i} / A_{i}, \operatorname{Con}\left(A_{i}\right)>\text { and } \mathcal{B}=<Y, D_{j} / B_{j}, \operatorname{Con}\left(B_{j}\right)>
$$

We will construct (non-canonical) Galois stratifications $\mathcal{A} \vee \mathcal{B}$ and $\mathcal{A} \wedge \mathcal{B}$ with the following property: for any point $x$ on $S$, and any field extension $M$ of $k(x)$,

$$
\begin{aligned}
Z(\mathcal{A} \vee \mathcal{B}, x, M) & =Z(\mathcal{A}, x, M) \cup Z(\mathcal{B}, x, M) \\
Z(\mathcal{A} \wedge \mathcal{B}, x, M) & =Z(\mathcal{A}, x, M) \cap Z(\mathcal{B}, x, M)
\end{aligned}
$$

After a refinement, we may assume that $\mathcal{A}$ and $\mathcal{B}$ have the same underlying stratification of $X$. After an inflation process, we may even assume that the underlying normal stratifications coincide. For any $i \in I$, we define the conjugation domain for $C_{i} / A_{i}$ in the Galois stratification $\mathcal{A} \vee \mathcal{B}$ (resp. $\mathcal{A} \wedge \mathcal{B}$ ) as the union (resp. the intersection) of the corresponding conjugation domains in $\mathcal{A}$ and $\mathcal{B}$.

3.8. Product of Galois stratifications. Let $X$ and $Y$ be varieties over $S$. Consider Galois stratifications

$$
\mathcal{A}=<X, C_{i} / A_{i}, \operatorname{Con}\left(A_{i}\right)>\text { and } \mathcal{B}=<Y, D_{j} / B_{j}, \operatorname{Con}\left(B_{j}\right)>
$$

We will construct a (non-canonical) Galois stratification $\mathcal{A} \times{ }_{S} \mathcal{B}$ of $X \times_{S} Y$, such that, for any point $x$ on $S$, and any field extension $M$ of $k(x)$, we have

$$
Z\left(\mathcal{A} \times{ }_{S} \mathcal{B}, x, M\right)=Z(\mathcal{A}, x, M) \times Z(\mathcal{B}, x, M) \subset\left(X \times{ }_{S} Y\right)_{x}(M)
$$

Denote by $\pi_{1}$ and $\pi_{2}$ the projections of $X \times_{S} Y$ on $X$, resp. $Y$. Pull back the Galois stratifications $\mathcal{A}$ and $\mathcal{B}$ to Galois stratifications $\mathcal{A}^{\prime}$ and $\mathcal{B}^{\prime}$ of $X \times_{S} Y$, via $\pi_{1}$ 
and $\pi_{2}$. After a refinement, we may assume that the underlying stratifications of $\mathcal{A}^{\prime}$ and $\mathcal{B}^{\prime}$ coincide. Let $U$ be a stratum. Let $C^{\prime} / U$ and $D^{\prime} / U$ be the Galois covers from $\mathcal{A}^{\prime}$, resp. $\mathcal{B}^{\prime}$, with conjugation domains $\operatorname{Con}\left(C^{\prime} / U\right)$ and $C o n\left(D^{\prime} / U\right)$. Let $V / U$ be any Galois cover dominating both $C^{\prime} / U$ and $D^{\prime} / U$. We define a conjugation domain $\operatorname{Con}(V)$ as the intersection of the inflations of $\operatorname{Con}\left(C^{\prime} / U\right)$ and $C o n\left(D^{\prime} / U\right)$ to $V / U$. The constructed Galois cover has the desired property.

3.9. Conjunction, disjunction and product of quantifier-free Galois formulas. Fix integers $m, n \geq 0$, consider Galois stratifications

$$
\mathcal{A}=<\mathbb{A}_{S}^{m}, C_{i} / A_{i}, \operatorname{Con}\left(A_{i}\right)>\text { and } \mathcal{B}=<\mathbb{A}_{S}^{n}, D_{j} / B_{j}, \operatorname{Con}\left(B_{j}\right)>
$$

and denote by $\theta_{\mathcal{A}}$ and $\theta_{\mathcal{B}}$ the corresponding Galois formulas.

If $m=n$, then we define $\theta_{\mathcal{A}} \vee \theta_{\mathcal{B}}$ and $\theta_{\mathcal{A}} \wedge \theta_{\mathcal{B}}$ as the Galois formulas corresponding to the Galois stratifications $\mathcal{A} \vee \mathcal{B}$, resp. $\mathcal{A} \wedge \mathcal{B}$.

For any $m, n$, we define $\theta_{\mathcal{A}} \times_{S} \theta_{\mathcal{B}}$ as the Galois formula corresponding to the Galois stratifications $\mathcal{A} \times{ }_{S} \mathcal{B}$.

The Galois formulas are not canonically defined, but the sets $Z(., x, M)$ they define are independent of any choices, for any point $x$ of $S$ and any field extension $M$ of $k(x)$.

\section{Quantifier elimination FOR Galois FORMulas}

Theorem 4.1 (Relative quantifier elimination). Let $S$ be a variety over a field $k$ of characteristic zero. Let $\mathcal{A}$ be a Galois stratification of $\mathbb{A}_{S}^{m+n}$, and let $\theta$ be a Galois formula

$$
\left(Q_{1} X_{1}\right) \ldots\left(Q_{m} X_{m}\right)[\mathcal{A} r(\mathbf{X}, \mathbf{Y}) \subset \operatorname{Con}(\mathcal{A})]
$$

There exists a Galois stratification $\mathcal{B}$ of $\mathbb{A}_{S}^{n}$ such that, for each point $x$ of $S$, and each pseudo-finite field $M$ containing $k(x)$,

$$
Z(\theta, x, M)=Z(\mathcal{B}, x, M)
$$

The absolute case $S=\operatorname{Spec} k$ was proven in [19]. The relative case can immediately be reduced to the absolute one, but we prefer to give a direct, purely geometric proof, the novelty being the use of the exact sequence of algebraic fundamental groups.

Theorem 4.1 follows from the elimination procedure in Lemma 4.3 and Lemma 4.4 below. First, we need an auxiliary result.

Lemma 4.2. Let $f: Y \rightarrow X$ be a proper, smooth morphism of irreducible $k$ varieties, let $V$ be a relative strict normal crossing divisor on $Y$ over $X^{2}$, put $U=Y \backslash V$, and let $g: Z \rightarrow U$ be a finite étale morphism of irreducible $k$-varieties. If $k(X)$ is algebraically closed in $k(Z)$, then the fibers of $h:=f \circ g$ are geometrically irreducible.

Proof. Let $z$ be any geometric point on $U$, and put $x=f \circ z$. We denote by $U_{x}$ and $Z_{x}$ the fibers of $U$, resp. $Z$, over $x$.

By 11, Exposé XIII, Lemme 4.1 and Exemples 4.4, there exists an exact homotopy sequence for the morphism $\left.f\right|_{U}: U \rightarrow X$ of the form

$$
\pi_{1}\left(U_{x}, z\right) \stackrel{f_{1}}{\longrightarrow} \pi_{1}(U, z) \stackrel{f_{2}}{\longrightarrow} \pi_{1}(X, x) \longrightarrow 1
$$

\footnotetext{
${ }^{2}$ The definition of a relative strict normal crossing divisor is recalled in Definition 5.2
} 
If $Z_{x}$ were not connected, we could find a non-trivial étale cover $E$ of $U$, dominated by $Z$, such that $E$ admits a section over $U_{x}$ : it suffices to take the quotient of $Z$ by the decomposition subgroup of $U_{x}$ at any connected component of $Z_{x}$ w.r.t. $Z / U$.

By [33, 5.2.5], the fact that $\operatorname{ker}\left(f_{2}\right)$ is contained in $\operatorname{Im}\left(f_{1}\right)$, has the following geometric interpretation: for any connected étale cover $E$ of $U$, such that the restriction over $U_{x}$ admits a section, there exists an étale cover $E^{\prime}$ of $X$, and a $U$-morphism from a connected component $C$ of $E^{\prime} \times_{X} U$ to $E$.

However, since $k(X)$ is algebraically closed in $k(Z)$, any such $E$ is trivial. Hence, $Z_{x}$ is connected.

Lemma 4.3 (Elimination of an existential quantifier). For every Galois stratification $\mathcal{A}$ of $\mathbb{A}_{S}^{m+1}$, there exists a Galois stratification $\mathcal{B}$ of $\mathbb{A}_{S}^{m}$, such that, for each point $x$ of $S$, for each pseudo-finite field extension $M$ of $k(x)$, and for each point $b$ of $\mathbb{A}_{x}^{m}(M)$, the condition $\mathcal{A} r(b) \subset C o n(\mathcal{B})$ is equivalent to

$$
\left(\exists a \in \mathbb{A}_{S}^{m+1}(M)\right)(\pi(a)=b \wedge \mathcal{A} r(a) \subset \operatorname{Con}(\mathcal{A}))
$$

where $\pi: \mathbb{A}_{S}^{m+1} \rightarrow \mathbb{A}_{S}^{m}$ is the projection on the first $m$ coordinates.

Proof. Let $\mathcal{A}$ be a Galois stratification of $\mathbb{A}_{S}^{m+1}$. Refining

$$
\mathcal{A}=<\mathbb{A}_{S}^{m+1}, C_{i} / A_{i}, \operatorname{Con}\left(A_{i}\right) \mid i \in I>
$$

if necessary, we may suppose that there exists a finite stratification $\left\{B_{j}\right\}_{j \in J}$ of $\mathbb{A}_{S}^{m}$ into normal, integral, locally closed subsets, such that for any $i \in I$, there exists an index $j(i) \in J$ such that $\pi: A_{i} \rightarrow \mathbb{A}_{S}^{m}$ factors through a smooth surjective morphism $\pi: A_{i} \rightarrow B_{j(i)}$. This stratification is constructed using generic smoothness and Noetherian induction.

For each $j \in J$, and each $i \in I$ with $j(i)=j$, we will construct a Galois cover $D_{j}^{i} / B_{j}$ and a conjugation domain $\operatorname{Con}\left(D_{j}^{i} / B_{j}\right)$ with the following property: for any pseudo-finite field $M$, and any $M$-valued point $b$ on $B_{j}, C_{D_{j}^{i} / B_{j}}(b) \subset C o n\left(D_{j}^{i} / B_{j}\right)$ iff there exists an $M$-valued point $a$ on $A_{i}$ with $\pi(a)=b$ and $C_{C_{i} / A_{i}}(a) \subset \operatorname{Con}\left(A_{i}\right)$. Dominating the covers $D_{j}^{i} / B_{j}$ by a common Galois cover $D_{j} / B_{j}$ and inflating the conjugation domains, we may suppose that all the covers $D_{j}^{i} / B_{j}$ coincide. Finally, we put $\operatorname{Con}\left(B_{j}\right):=\cup_{i \in I, j(i)=j} \operatorname{Con}\left(D_{j}^{i} / B_{j}\right)$, and

$$
\mathcal{B}:=<\mathbb{A}_{S}^{m}, D_{j} / B_{j}, \operatorname{Con}\left(B_{j}\right)>
$$

The Galois stratification $\mathcal{B}$ satisfies the requirements.

We fix $j \in J$, and $i \in I$ with $j=j(i)$. We'll simply write $C / A, D / B$ and $\operatorname{Con}(B)$, instead of $C_{i} / A_{i}, D_{j}^{i} / B_{j}$ and $\operatorname{Con}\left(D_{j}^{i} / B_{j}\right)$.

We will distinguish two cases: either the dimensions of $A$ and $B$ agree, or $\operatorname{dim} A=$ $\operatorname{dim} B+1$.

Case 1: $\operatorname{dim} A=\operatorname{dim} B$. Stratifying $B$ if necessary, we may suppose that $\pi: A \rightarrow$ $B$ is étale and finite. Hence, $C$ is an étale cover of $B$, and can be dominated by a Galois cover $D \rightarrow B$. We define $C o n(B)^{\prime}$ as the inflation of $C o n(A)$ to $D / A$, and $\operatorname{Con}(B)$ as the smallest conjugation domain in $G(D / A)$ containing $C o n(B)^{\prime}$. Here we consider $G(D / A)$ as a subgroup of $G(D / B)$.

Case 2: $\operatorname{dim} A=\operatorname{dim} B+1$. Then $\pi: A \rightarrow B$ factors through an open immersion $A \rightarrow \operatorname{Spec} \mathcal{O}_{B}[t]$.

If we denote by $D$ the normalization of $B$ in the algebraic closure of $k(B)$ in $k(C)$, then $D$ is a Galois cover of $B$. Intuitively, this construction extracts the 
Galois action on the base $B$ from the cover $C / A$. The cover $C / A$ factors through the Galois cover $A \times_{B} D \rightarrow A$, and

$$
G\left(A \times_{B} D / A\right) \cong G(D / B)
$$

Let $C o n(B)$ be the conjugation domain, obtained by restricting the elements of the members of $C o n(A)$ to $G(D / B)$ via

$$
G(C / A) \rightarrow G\left(A \times_{B} D / A\right) \cong G(D / B)
$$

Let $x$ be a point of $S$, let $M$ be a field extension of $k(x)$, and let $b$ be a point of $B_{x}(M)$. First, suppose that there exists a point $a$ of $A(M)$, with $\pi(a)=b$, such that $C_{C / A}(a) \subset C o n(A)$. Since $C_{D / B}(b)$ is obtained from $C_{C / A}(a)$ by restriction (by Lemma 2.6 and the isomorphism (2)), $C_{D / B}(b) \subset \operatorname{Con}(B)$.

Now suppose, conversely, that $C_{D / B}(b) \subset \operatorname{Con}(B)$, and that $M$ is pseudo-finite. Let $b^{\prime}$ be a point on the fiber of $D / B$ over $b$. Its decomposition group is an element $H_{0}$ of $\operatorname{Con}(B)$. We denote its residue field $k\left(b^{\prime}\right)$ by $N$.

Put $F_{b^{\prime}}=A \times_{B} b^{\prime}$, and let $C_{b^{\prime}}$ be the inverse image of $F_{b^{\prime}}$ under the Galois cover $C / A \times{ }_{B} D$. Stratifying the base $B$, we may suppose that the complement of $A$ in Spec $\mathcal{O}_{B}[t]$ is either empty, or étale and finite over $B$. This means, in particular, that $A \times{ }_{B} D$ is the complement in $D \times \mathbb{P}^{1}$ of a divisor with normal crossings, relative to $D$. By Lemma 4.2 this implies that $C_{b^{\prime}}$ is geometrically connected over $k\left(b^{\prime}\right)$. Hence, the decomposition group $D_{C / A \times_{B} D}\left(C_{b^{\prime}} / F_{b^{\prime}}\right)$ is the whole Galois group $G\left(C / A \times_{B} D\right)$, i.e. the Galois action of $C / A \times_{B} D$ is concentrated on the fibers.

By definition of $C o n(B)$, there exists an element $H_{1}$ of $C o n(A)$, such that $H_{0}$ is the image of $H_{1}$ under the restriction morphism $G(C / A) \rightarrow G(D / B)$. Let $F_{b}$ be the fiber of $A$ over $b$, and consider the induced Galois cover $C_{b^{\prime}} / F_{b}$. Since $C_{b^{\prime}}$ is geometrically connected over $k\left(b^{\prime}\right)$, the algebraic closure of $M$ in $k\left(C_{b^{\prime}}\right)$ is $N$. By Lemma 2.7 there exists a point $a$ of $F_{b}(M)$ with decomposition group $H_{1} \in \operatorname{Con}(A)$.

Lemma 4.4 (Elimination of a universal quantifier). For every Galois stratification $\mathcal{A}$ of $\mathbb{A}_{S}^{m+1}$, there exists a Galois stratification $\mathcal{B}$ of $\mathbb{A}_{S}^{m}$, such that, for each point $x$ of $S$, for each pseudo-finite field extension $M$ of $k(x)$, and for each point $b$ of $\mathbb{A}_{x}^{m}(M)$, the condition $\mathcal{A} r(b) \subset C o n(\mathcal{B})$ is equivalent to

$$
\left(\forall a \in \mathbb{A}_{S}^{m+1}(M)\right)(\pi(a)=b \Rightarrow \mathcal{A} r(a) \subset \operatorname{Con}(\mathcal{A}))
$$

Proof. We will deduce Lemma 4.4 from Lemma 4.3 Let $\mathcal{A}^{c}$ be the complementary Galois stratification of $\mathcal{A}$; this means that $\mathcal{A}^{c}$ has the same underlying normal stratification, but for each stratum $A_{i}$, the conjugation domain $\operatorname{Con}^{c}\left(A_{i}\right)$ associated to $A_{i}$ by $\mathcal{A}^{c}$ consists of the cyclic subgroups of $G\left(C_{i} / A_{i}\right)$ that do not belong to $\operatorname{Con}\left(A_{i}\right)$. Lemma 4.3 produces a Galois stratification $\mathcal{B}^{c}$ of $\mathbb{A}_{S}^{m}$; its complement $\mathcal{B}$ satisfies the requirements.

Repeatedly applying Lemma 4.3 and Lemma 4.4 proves Theorem 4.1

Corollary 4.5. Let $S$ be an irreducible $k$-variety, and let $\varphi_{1}, \varphi_{2}, \psi$ be ring formulas over $S$. Then $\psi$ defines an equivalence of $\varphi_{1}$ and $\varphi_{2}$ over the generic point $\eta$ of $S$, iff $\psi$ defines an equivalence of $\varphi_{1}$ and $\varphi_{2}$ over each closed point $x$ in a dense open subset $U$ of $S$. 
Proof. The property ' $\psi$ defines a bijection between $\varphi_{1}$ and $\varphi_{2}$ ' is definable by a sentence $\theta$, i.e. a ring formula over $S$ without free variables. Hence, it suffices to show that $\theta$ is true in every pseudo-finite extension of $k(\eta)$, iff it is true in every pseudo-finite extension of $k(x)$, for each closed point $x$ in some dense open subset $U$ of $S$.

By the elimination theorem, we may suppose that $\theta$ is a Galois formula without quantifiers and without free variables. Let $U$ be the stratum of the corresponding Galois stratification, containing $\eta$. Let $C / U$ be the associated Galois cover, and $C o n(U)$ its conjugation domain.

The interpretation of $\theta$ is true, in every pseudo-finite extension of $k(\eta)$, iff $C o n(U)$ contains the decomposition classes of $C / U$ at all pseudo-finite extensions of $k(\eta)$. By Lemma 2.8, applied to the Galois cover $k(C) / k(\eta)$, this is the case iff $C o n(U)$ contains all cyclic subgroups of $G(C / U)$. Again by Lemma 2.8 this is, at its turn, equivalent to saying that the interpretation of $\theta$ is true, in every pseudo-finite extension of every closed point $x$ of $U$.

Corollary 4.6. As an Abelian group, the Grothendieck ring $K_{0}\left(P F F_{S}\right)$ is generated by classes of the form $\left[\varphi_{Y / X, C}\right]$, where $Y / X$ is a Galois cover of affine normal irreducible $S$-varieties, and $C$ is a cyclic subgroup of $G(Y / X)$ (see Section 3.3 for the definition of the formula $\left.\varphi_{Y / X, C}\right)$.

Proof. By the elimination theorem, $K_{0}\left(P F F_{S}\right)$ is generated by the classes of (ring formulas corresponding to) quantifier-free Galois formulas $\theta$. Writing the conjugation domain of $\theta$ as a union of conjugation classes of cyclic groups, we see that the class of $\theta$ in $K_{0}\left(P F F_{S}\right)$ can be written as a sum of classes of the form $\left[\varphi_{Y / X, C}\right]$.

\section{Constructible Resolution of singularities}

5.1. Varieties with good group action. Let $S$ be a variety over a field $k$ of characteristic zero, and let $G$ be a finite group. If $X$ is any variety over $S$, a good $G$-action on $X$ is an action of $G$ on $X$, such that the structural morphism $X \rightarrow S$ is equivariant (where $S$ carries the trivial action), and such that every orbit is contained in an affine open subscheme of $X$ (this is automatic if $X$ is quasiprojective over $S)$. We define the Grothendieck group $K_{0}^{G}\left(\operatorname{Var}_{S}\right)$ as follows: start with the free abelian group on isomorphism classes $[X]$ of $S$-varieties $X$ with good $G$-action, and consider relations of the form $[X]=[Z]+[X \backslash Z]$, where $Z$ is a $G$-invariant closed subvariety with the induced $G$-action. The fiber product over $S$ induces a ring product on $K_{0}^{G}\left(\operatorname{Var}_{S}\right)$. If $X$ is an $S$-variety, and $S_{i}$ is a locally closed subset of $S$, we denote by $X_{S_{i}}$ the $S_{i}$-variety $\left(X \times{ }_{S} S_{i}\right)_{\text {red }}$.

For any morphism of $k$-varieties $f: S^{\prime} \rightarrow S$, there is a base-change morphism of rings

$$
f^{*}: K_{0}^{G}\left(\operatorname{Var}_{S}\right) \rightarrow K_{0}^{G}\left(\operatorname{Var}_{S^{\prime}}\right)
$$

For any morphism of finite groups $G \rightarrow G^{\prime}$, there is a forgetful morphism

$$
K_{0}^{G^{\prime}}\left(\operatorname{Var}_{S}\right) \rightarrow K_{0}^{G}\left(\operatorname{Var}_{S}\right)
$$

If $G$ is the trivial group $\{e\}$, we write $K_{0}\left(\operatorname{Var}_{S}\right)$ instead of $K_{0}^{G}\left(\operatorname{Var}_{S}\right)$.

5.2. Resolution of singularities. Let $S$ be a variety over a field $k$ of characteristic zero. 
Definition 5.1. An admissible stratification is a finite stratification $\mathscr{S}=\left\{S_{1}, \ldots, S_{p}\right\}$ of $S$ into smooth, irreducible, quasi-projective, locally closed subvarieties $S_{i}$.

Definition 5.2 (1, XIII.2.1). Let $X$ be a smooth $S$-variety. A strict normal crossing divisor relative to $S$ on $X$ is a divisor on $X$, with the following properties:

- the prime components of $D$ are smooth over $S$,

- at any point $x$ on $X$, these components are locally defined by $f_{i}=0, i=$ $1, \ldots, r$, with $f_{i}(x)=0$. The scheme $V\left(f_{1}, \ldots, f_{r}\right)$ is smooth over $S$, of codimension $r$ in $X$.

Definition 5.3. A resolution of singularities for a variety $X$ over $S$, is a composition of blow-ups with $S$-smooth centers $h: X^{\prime} \rightarrow X$, such that $X^{\prime}$ is smooth over $S$, and such that the exceptional locus $E$ of $h$ is a strict normal crossing divisor relative to $S$.

If $S=$ Spec $k$, any variety $X$ over $S$ admits a resolution of singularities, by Hironaka's famous result 28. This does not hold for arbitrary $S$. The following result is a constructible version of resolution of singularities over $S$.

Proposition 5.4 (Constructible resolution of singularities). For any variety $X$ over $S$, there exists an admissible stratification $\mathscr{S}=\left\{S_{i} \mid i \in I\right\}$ of $S$, such that for any $i \in I$, the variety $\left(X \times{ }_{S} S_{i}\right)_{\text {red }}$ admits a resolution of singularities over $S_{i}$.

If $G$ is a finite group, and $X$ carries a good $G$-action, then all the blow-ups in the resolution can be chosen to have $G$-closed centers, so that the resolution of singularities is $G$-equivariant. Moreover, if $V$ is a $G$-closed, closed subvariety of $X$, we can find a resolution by blow-ups of smooth $G$-closed centers, such that the union of the exceptional locus with the inverse image of $V$ is a strict normal crossing divisor relative to $S$.

Proof. It suffices to prove that there exists a non-empty open subvariety $U$ of $S$, such that $X \times{ }_{S} U$ admits a resolution over $U$ by blow-ups with $G$-closed centers. The theorem then follows by Noetherian induction on $S$.

By $G$-equivariant uniformization of ideals [36] $\S 7]$, the result holds for the fiber $X_{\eta}$ of $X$ over the generic point $\eta$ of $S$. Hence, it suffices to prove the following claims.

1. If $X_{\eta}$ is smooth over $\eta$, then there exists a non-empty open subscheme $U$ of $X$, such that $X_{U}$ is smooth over $U$.

We may assume that $X$ and $S$ are affine. If the Jacobian criterion for smoothness holds over $\eta$, it holds over an open neighbourhood $U$ of $\eta$ in $S$.

2. If $Z_{\eta}$ is a closed smooth subvariety of $X_{\eta}$, then there exists a non-empty open subscheme $U$ of $S$, and a smooth closed subvariety $Z_{U}$ of $X_{U}:=X \times{ }_{S} U$, such that $Z_{\eta}$ is the fiber of $Z_{U}$ over $\eta$. The blow-up of $X_{\eta}$ at $Z_{\eta}$ is the fiber over $\eta$ of the blow-up of $X_{U}$ at $Z_{U}$.

Working locally, we may assume that $S$ and $X$ are affine. The equations defining $Z_{\eta}$ in $X_{\eta}$ extend to regular functions on $X_{U}$, for some non-empty open subscheme $U$ of $X$, and these define a closed subvariety $Z_{U}$ with $Z_{\eta}=Z_{U} \times_{U} \eta$. By point 1 , after shrinking $U$, we may assume that $Z_{U}$ is smooth over $U$. The last part of the claim follows from flat base change for blow-ups [30, 8.1.12.c].

3. If $D$ is a divisor on $X$, and $D_{\eta}:=D \times_{X} \eta$ is a strict normal crossing divisor on $X_{\eta}$, then there exists a non-empty open subscheme $U$ of $S$ such that $D_{U}:=D \times_{X} U$ is a strict normal crossing divisor relative to $U$ on $X_{U}$. 
J. NICAISE

Apply point 1 to the components of $D$ and their intersections.

Proposition 5.5 (Constructible Chow-Hironaka Lemma). Let $G$ be a finite group. Let $X, X^{\prime}$ be smooth irreducible $S$-varieties of relative dimension d, carrying a good $G$-action, and let $Y$ be a closed subvariety of $X^{\prime}$, such that the dimensions of the fibers of $Y \rightarrow S$ are strictly smaller than $d$. Let $h: X^{\prime} \rightarrow X$ be a G-equivariant proper birational morphism over $S$, such that $h$ is an isomorphism over $X \backslash Y$.

There exists an admissible stratification $\mathscr{S}=\left\{S_{i} \mid i \in I\right\}$ such that, for any $i \in I$, the morphism $h_{S_{i}}: X_{S_{i}}^{\prime} \rightarrow X_{S_{i}}$ can be dominated by a composition of blow-ups with $S_{i}$-smooth, G-closed centers.

Proof. By the equivariant Chow-Hironaka Lemma, the proposition holds if $S$ is the spectrum of a field (see 11, Lemma A.2). Now we can proceed as in the proof of Proposition 5.4

Proposition 5.6 (Constructible compactification). Let $G$ be a finite group. Let $X$ be a smooth $S$-variety, carrying a good $G$-action, and let $V$ be a $G$-closed, closed subvariety. There exists an admissible stratification $\mathscr{S}=\left\{S_{i} \mid i \in I\right\}$ such that, for any $i \in I$, we can find a G-equivariant compactification $X_{S_{i}} \hookrightarrow X_{S_{i}}^{\prime}$, with $X_{S_{i}}^{\prime}$ a smooth, proper $S_{i}$-variety with good $G$-action, and such that the union of $X_{S_{i}}^{\prime} \backslash X_{S_{i}}$ with the closure of $V_{S_{i}}$ is a strict normal crossing divisor relative to $S_{i}$.

Proof. Starting with a $G$-equivariant compactification of $X$ over $S$, we can use Proposition 5.4 to obtain the result.

\section{Constructible Chow motives over a Base variety}

For the categorical language in this section (additive and pseudo-abelian categories, tensor structures, ...) we refer to [29].

6.1. Pseudo-abelian categories. An additive category $\mathcal{A}$ is called pseudo-abelian, if all projectors (i.e. all idempotent endomorphisms) split. This means that, for any projector $p$ on an object $A$, we can find an isomorphism $A \cong B \oplus C$ such that $p$ corresponds to $(I d, 0)$ on $B \oplus C$. In this case, the natural morphism $C \rightarrow A$ is a kernel for $p$, and $B \rightarrow A$ is an image. For any additive category $\mathcal{A}$, the pseudoabelian envelope is an additive full embedding $\mathcal{A} \rightarrow \mathcal{A}_{\sharp}$ defined by the following universal property: $\mathcal{A}_{\sharp}$ is pseudo-abelian, and any additive functor from $\mathcal{A}$ to a pseudo-abelian category $\mathcal{C}$ factors through an essentially unique additive functor from $\mathcal{A}_{\sharp}$ to $\mathcal{C}$.

The pseudo-abelian envelope $\mathcal{A}_{\sharp}$ of an additive category $\mathcal{A}$ is constructed by artificially adding images for projectors (we get kernels for free, since the kernel of a projector $p$ coincides with the image of $I d-p)$. The objects of $\mathcal{A}_{\sharp}$ are pairs $(A, p)$, where $A$ is an object of $\mathcal{A}$ and $p$ is a projector on $A$. The morphisms are given by

$$
\operatorname{Hom}_{\mathcal{A}_{\sharp}}((A, p),(B, q)):=q \circ \operatorname{Hom}_{\mathcal{A}}(A, B) \circ p
$$

\subsection{The Grothendieck ring of an additive category.}

Definition 6.1 (Grothendieck ring). For any additive category $\mathcal{A}$, the Grothendieck group $K_{0}(\mathcal{A})$ is the abelian group generated by the monoid $([\mathcal{A}], \oplus)$, where $[\mathcal{A}]$ denotes the set ${ }^{3}$ of isomorphism classes of objects of $\mathcal{A}$, and $\oplus$ is the direct sum. We denote the class of an object $A$ of $\mathcal{A}$ in $K_{0}(\mathcal{A})$ by $[A]$.

\footnotetext{
${ }^{3}$ We tacitly assume that the isomorphism classes of objects of $\mathcal{A}$ form a set.
} 
If $\mathcal{A}$ carries a tensor structure, the tensor product $\otimes$ induces a multiplication on $K_{0}(\mathcal{A})$, determined by $[A] \cdot[B]:=[A \otimes B]$. This multiplication turns $K_{0}(\mathcal{A})$ into a ring: the Grothendieck ring associated to $\mathcal{A}$.

An additive functor $F: \mathcal{A} \rightarrow \mathcal{B}$ respects direct sums, and hence induces a morphism of Abelian groups $K_{0}(F): K_{0}(\mathcal{A}) \rightarrow K_{0}(\mathcal{B})$. If $\mathcal{A}$ and $\mathcal{B}$ carry tensor structures, and $F$ respects tensor structures, $K_{0}(F)$ is a morphism of rings.

Remark. This construction is not to be confused with the definition of the Grothendieck group of an exact category (the free abelian group on isomorphism classes, modulo the relation $[B]=[A]+[C]$ whenever $0 \rightarrow A \rightarrow B \rightarrow C \rightarrow 0$ is a short exact sequence). For instance; consider the category $\mathcal{A} b_{f g}$ of finitely generated abelian groups. Any such group $G$ can be written canonically as a direct sum of $\mathbb{Z}^{r}$ for some integer $r>0$, and a finite number of torsion groups $\mathbb{Z} /\left(p^{i}\right)$, with $p$ a prime. The rank map

$$
r k: \operatorname{Ob}(\mathcal{A}) \rightarrow \mathbb{Z}: G \mapsto r
$$

induces an isomorphism from the Grothendieck group of the abelian category $\mathcal{A} b_{f g}$ to $\mathbb{Z}$ : torsion parts are killed, by the existence of an exact sequence $0 \rightarrow \mathbb{Z} \rightarrow$ $\mathbb{Z} \rightarrow \mathbb{Z} /(n) \rightarrow 0$ for any integer $n>0$. However, if we consider $\mathcal{A} b_{f g}$ merely as an additive category, we can write any element $[G]$ of $K_{0}\left(\mathcal{A} b_{f g}\right)$ uniquely as

$$
r[\mathbb{Z}]+\sum_{p>0 \text { prime }, i>0} n_{p, i}\left[\mathbb{Z} /\left(p^{i}\right)\right]
$$

with $r, n_{p, i}$ integers, and $n_{p, i}=0$ for almost all $p$ and $i$.

6.3. Effective Chow motives over a smooth base. Let $k$ be a field of characteristic zero, and let $S$ be a smooth irreducible quasi-projective variety over $k$. We briefly recall the construction of the category $\mathcal{M}_{+}^{o}(S)$ of Chow motives over $S$ (see [17. §1]), and we prove some elementary properties.

Denote by $\mathcal{V}_{S}$ the category of smooth, projective varieties $X$ over $S$. If $X$ is irreducible, we denote the relative dimension of $X$ over $S$ by $d(X / S)$. For any smooth projective variety $X$ over $S$, and any integer $\alpha \geq 0$, we denote by $C H^{\alpha}(X)$ the Chow group of algebraic cycles of degree $\alpha$. It is constructed as follows: consider the free abelian group on the set of irreducible subvarieties of $X$ of codimension $\alpha$, and take the quotient modulo rational equivalence. We put $C H^{\alpha}(X, \mathbb{Q}):=$ $C H^{\alpha}(X) \otimes_{\mathbb{Z}} \mathbb{Q}$. For any pair of integers $\alpha, \beta \geq 0$, there is a bilinear intersection pairing

$$
C H^{\alpha}(X, \mathbb{Q}) \times C H^{\beta}(X, \mathbb{Q}) \rightarrow C H^{\alpha+\beta}(X, \mathbb{Q}):(x, y) \mapsto x . y
$$

which makes the graded group $C H(X, \mathbb{Q}):=\oplus_{\alpha} C H^{\alpha}(X, \mathbb{Q})$ into a ring.

If $X, Y$ and $Z$ are smooth, projective varieties over $S$, with $X$ and $Y$ irreducible, we can construct a bilinear map

$$
\circ: C H^{d(X / S)}\left(X \times{ }_{S} Y, \mathbb{Q}\right) \times C H^{d(Y / S)}\left(Y \times{ }_{S} Z, \mathbb{Q}\right) \rightarrow C H^{d(X / S)}\left(X \times_{S} Z, \mathbb{Q}\right)
$$

as follows: a couple $(f, g)$ is mapped to

$$
f \circ g:=\left(p_{13}\right)_{*}\left(p_{12}^{*}(f) \cdot p_{23}^{*}(g)\right)
$$

where $p_{12}, p_{13}, p_{23}$ are the projections of $X \times_{S} Y \times_{S} Z$ on $X \times_{S} Y, X \times_{S} Z$, resp. $Y \times_{S} Z$, and "." is the intersection product in the Chow ring of $X \times_{S} Y \times_{S} Z$. 
Let $C \mathcal{V}_{S}^{o}$ be the category with the same objects as $\mathcal{V}_{S}$, but with morphisms

$$
H_{C m_{C} \mathcal{V}_{S}^{\circ}}(X, Y):=\oplus_{X_{i} \in \mathcal{C}(X)} C H^{d\left(X_{i} / S\right)}\left(X_{i} \times_{S} Y, \mathbb{Q}\right)
$$

where $\mathcal{C}(X)$ is the set of connected components of $X$. The bilinear map o constructed above defines the composition law in $C \mathcal{V}_{S}^{o}$, turning it into an additive category with $\mathbb{Q}$-linear structure; the direct sum $X \oplus Y$ is simply the disjoint union $X \sqcup Y$. There is a canonical contravariant functor

$$
M: \mathcal{V}_{S} \rightarrow C \mathcal{V}_{S}^{o}
$$

mapping a $S$-morphism $f: Y \rightarrow X$ to the transpose of its graph.

The category $\mathcal{M}_{+}^{o}(S)$ of (effective) Chow motives over $S$, is defined as the pseudoabelian envelope of $C \mathcal{V}_{S}^{o}$. The fiber product over $S$ induces a tensor structure on $\mathcal{M}_{+}^{o}(S)$. The functor $M$ induces a canonical contravariant functor

$$
M: \mathcal{V}_{S} \rightarrow \mathcal{M}_{+}^{o}(S)
$$

which we will denote by $M_{S}$ if we want to make the base explicit. To simplify notation, we will sometimes denote the image of a morphism of smooth projective $S$-varieties $f: X \rightarrow Y$ by $f^{*} \in H_{o m} \mathcal{M}_{+}^{o}(S)(M(Y), M(X))$. This notation is not to be confused with the base change functor defined below.

Applying Definition 6.1 to the additive tensor category $\mathcal{M}_{+}^{o}(S)$, we obtain the Grothendieck ring of Chow motives $K_{0}\left(\mathcal{M}_{+}^{o}(S)\right)$.

If $f: S^{\prime} \rightarrow S$ is a morphism of smooth, irreducible, quasi-projective $k$-varieties, then the base change functor $f^{*}: \mathcal{V}_{S} \rightarrow \mathcal{V}_{S^{\prime}}$ induces a $\mathbb{Q}$-linear base change functor

$$
f^{*}: \mathcal{M}_{+}^{o}(S) \rightarrow \mathcal{M}_{+}^{o}\left(S^{\prime}\right)
$$

compatible with the tensor structures, and a ring morphism

$$
f^{*}: K_{0}\left(\mathcal{M}_{+}^{o}(S)\right) \rightarrow K_{0}\left(\mathcal{M}_{+}^{o}\left(S^{\prime}\right)\right)
$$

Lemma 6.2. If $f: S^{\prime} \rightarrow S$ is a smooth, projective morphism of smooth, irreducible, quasi-projective $k$-varieties, then the forgetful functor $f_{*}: \mathcal{V}_{S^{\prime}} \rightarrow \mathcal{V}_{S}$ induces a $\mathbb{Q}$ linear forgetful functor

$$
f_{*}: \mathcal{M}_{+}^{o}\left(S^{\prime}\right) \rightarrow \mathcal{M}_{+}^{o}(S)
$$

(generally not compatible with the tensor structures), and a morphism of abelian groups

$$
f_{*}: K_{0}\left(\mathcal{M}_{+}^{o}\left(S^{\prime}\right)\right) \rightarrow K_{0}\left(\mathcal{M}_{+}^{o}(S)\right)
$$

Proof. Let $X, Y$ be smooth and projective varieties over $S^{\prime}$. The canonical morphism $\pi: X \times \times_{S^{\prime}} Y \rightarrow X \times{ }_{S} Y$ is proper, and induces a degree $\left(\operatorname{dim}\left(S^{\prime}\right)-\operatorname{dim}(S)\right)$ morphism of graded $\mathbb{Q}$-vector spaces

$$
\pi_{*}: C H\left(X \times_{S^{\prime}} Y, \mathbb{Q}\right) \rightarrow C H\left(X \times{ }_{S} Y, \mathbb{Q}\right)
$$

Let us show that these morphisms are compatible with the composition of correspondences. Let $Z$ be another smooth projective $S^{\prime}$-variety, and let $\alpha$ and $\beta$ be elements of $C H\left(X \times_{S^{\prime}} Y, \mathbb{Q}\right)$, resp. $C H\left(Y \times_{S^{\prime}} Z, \mathbb{Q}\right)$. 
Consider the following diagram of Cartesian squares.

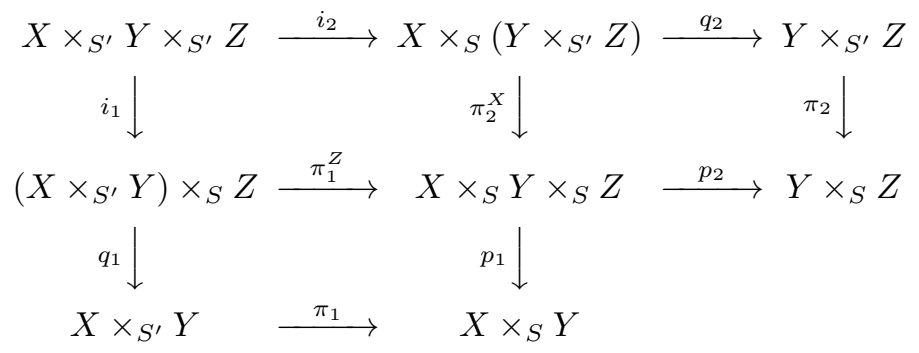

and denote by $p_{X Z}$ the projection of $X \times_{S} Y \times_{S} Z$ on $X \times_{S} Z$, and by $p_{X Z}^{\prime}$ the projection of $X \times{ }_{S^{\prime}} Y \times{ }_{S^{\prime}} Z$ on $X \times{ }_{S^{\prime}} Z$. Finally, we denote by

$$
\pi_{*}: C H\left(X \times{ }_{S^{\prime}} Z, \mathbb{Q}\right) \rightarrow C H\left(X \times{ }_{S} Z, \mathbb{Q}\right)
$$

the proper push-forward induced by $\pi: X \times{ }_{S^{\prime}} Z \rightarrow X \times{ }_{S} Z$.

Using [21, 1.7] (base change of the flat pull-back) and [21, 8.3(c)] (projection formula), we get

$$
\begin{aligned}
& \pi_{*}\left(p_{X Z}^{\prime}\right)_{*}\left[\left(i_{1}\right)^{*}\left(q_{1}\right)^{*}(\alpha) \cdot\left(i_{2}\right)^{*}\left(q_{2}\right)^{*}(\beta)\right] \\
= & \left(p_{X Z}\right)_{*}\left(\pi_{1}^{Z}\right)_{*}\left(i_{1}\right)_{*}\left[\left(i_{1}\right)^{*}\left(q_{1}\right)^{*}(\alpha) \cdot\left(i_{2}\right)^{*}\left(q_{2}\right)^{*}(\beta)\right] \\
= & \left(p_{X Z}\right)_{*}\left(\pi_{1}^{Z}\right)_{*}\left[\left(q_{1}\right)^{*}(\alpha) \cdot\left(i_{1}\right)_{*}\left(i_{2}\right)^{*}\left(q_{2}\right)^{*}(\beta)\right] \\
= & \left(p_{X Z}\right)_{*}\left(\pi_{1}^{Z}\right)_{*}\left[\left(q_{1}\right)^{*}(\alpha) \cdot\left(\pi_{1}^{Z}\right)^{*}\left(\pi_{2}^{X}\right)_{*}\left(q_{2}\right)^{*}(\beta)\right] \\
= & \left(p_{X Z}\right)_{*}\left[\left(\pi_{1}^{Z}\right)_{*}\left(q_{1}\right)^{*}(\alpha) \cdot\left(\pi_{2}^{X}\right)_{*}\left(q_{2}\right)^{*}(\beta)\right] \\
= & \left(p_{X Z}\right)_{*}\left[\left(p_{1}\right)^{*}\left(\pi_{1}\right)_{*}(\alpha) \cdot\left(p_{2}\right)^{*}\left(\pi_{2}\right)_{*}(\beta)\right]
\end{aligned}
$$

in $C H\left(X \times_{S} \times Y \times Z\right.$, Q ). If $\alpha$ and $\beta$ belong to $\operatorname{Hom}_{\mathcal{M}_{+}^{o}\left(S^{\prime}\right)}\left(M_{S^{\prime}}(X), M_{S^{\prime}}(Y)\right)$, resp. $\operatorname{Hom}_{\mathcal{M}_{+}^{o}\left(S^{\prime}\right)}\left(M_{S^{\prime}}(Y), M_{S^{\prime}}(Z)\right)$, then the first of these expressions is the image of $\beta \circ \alpha$ under $\pi_{*}$, while the last one is the composition of the images of $\alpha$ and $\beta$ in $\operatorname{Hom}_{\mathcal{M}_{+}^{o}(S)}\left(M_{S}(X), M_{S}(Y)\right)$, resp. $\operatorname{Hom}_{\mathcal{M}_{+}^{o}(S)}\left(M_{S}(Y), M_{S}(Z)\right)$.

Hence, we obtain a $\mathbb{Q}$-linear forgetful functor

$$
C \mathcal{V}_{S^{\prime}}^{o} \rightarrow C \mathcal{V}_{S}^{o}
$$

which passes to a $\mathbb{Q}$-linear forgetful functor

$$
f_{*}: \mathcal{M}_{+}^{o}\left(S^{\prime}\right) \rightarrow \mathcal{M}_{+}^{o}(S)
$$

by the universal property of the pseudo-abelian envelope.

Observe that $f_{*} \circ f^{*}=M\left(S^{\prime}\right) \otimes($.$) .$

We denote by $D^{b}\left(S, \mathbb{Q}_{\ell}\right)$ the derived category of bounded complexes of $\mathbb{Q}_{\ell}$-sheaves on $S$, in the sense of [18. The functor $\mathcal{V}_{S} \rightarrow D^{b}\left(S, \mathbb{Q}_{\ell}\right)$ mapping an object $h: X \rightarrow$ $S$ to $R h_{*}\left(\mathbb{Q}_{\ell}\right)$ extends to a $\mathbb{Q}$-linear realization functor

$$
\text { real }: \mathcal{M}_{+}^{o}(S) \rightarrow D^{b}\left(S, \mathbb{Q}_{\ell}\right)
$$

By the relative Künneth isomorphism, it respects the tensor structures: there is a canonical quasi-isomorphism

$$
\operatorname{real}\left(M_{1} \otimes M_{2}\right) \cong \operatorname{real}\left(M_{1}\right) \otimes^{L} \operatorname{real}\left(M_{2}\right)
$$


Definition 6.3 (Lefschetz motive). Let $e: S \rightarrow \mathbb{P}_{S}^{1}$ be any section of the structural morphism $\mathbb{P}_{S}^{1} \rightarrow S$. The cycle $\mathbb{P}_{S}^{1} \times_{S} e(S)$ defines a projector $p$ on $M\left(\mathbb{P}_{S}^{1}\right)$ in the category $\mathcal{M}_{+}^{o}(S)$, independent of the choice of e. The image of this projector is called the Lefschetz motive over $S$, and is denoted by $\mathbb{L}_{S}$.

If $S^{\prime} \rightarrow S$ is a morphism of smooth quasi-projective varieties over $k$, then the base-change functor $\mathcal{M}_{+}^{o}(S) \rightarrow \mathcal{M}_{+}^{o}\left(S^{\prime}\right)$ maps $\mathbb{L}_{S}$ to $\mathbb{L}_{S^{\prime}}$. We will simply write $\mathbb{L}_{S}^{d}$ for $\mathbb{L}_{S}^{\otimes d}$, for any integer $d \geq 0$. If $e_{d}: S \rightarrow \mathbb{P}_{S}^{d}$ is a section, we can define a projector $p_{d}=\mathbb{P}_{S}^{d} \times_{S} e_{d}(S)$ on $M\left(\mathbb{P}_{S}^{d}\right)$ in the category $\mathcal{M}_{+}^{o}(S)$, independent of the choice of $e_{d}$. Its image is isomorphic to $\mathbb{L}_{S}^{d}$, by the isomorphism

$$
\mathbb{P}_{S}^{d} \times_{S} e(S)^{\otimes d} \in \operatorname{Hom}_{\mathcal{M}_{+}^{o}(S)}\left(\left(M\left(\mathbb{P}_{S}^{d}\right), p_{d}\right), \mathbb{L}_{S}^{d}\right)
$$

Lemma 6.4. For any pair of objects $X, Y$ in $\mathcal{V}_{S}$, with $X$ of pure dimension $d$ over $S$, and for any pair of integers $r, s \geq 0$, there is a canonical isomorphism of $\mathbb{Q}$-vector spaces

$$
C H^{d+r-s}\left(X \times_{S} Y, \mathbb{Q}\right) \cong \operatorname{Hom}_{\mathcal{M}_{+}^{o}(S)}\left(M(X) \otimes \mathbb{L}_{S}^{r}, M(Y) \otimes \mathbb{L}_{S}^{s}\right)
$$

where we put $C H^{j}()=$.0 if $j<0$.

Proof. The functor

$$
(.) \otimes \mathbb{L}_{S}: \mathcal{M}_{+}^{o}(S) \rightarrow \mathcal{M}_{+}^{o}(S)
$$

is fully faithful, by [17, 1.6], so we may assume that $r=0$ or $s=0$. For any integer $i>0$, we'll denote by $f: X \times_{S} Y \times_{S} \mathbb{P}_{S}^{i} \rightarrow X \times_{S} Y$ the projection morphism.

First, suppose $s=0$. An element of $\operatorname{Hom}_{\mathcal{M}_{+}^{o}(S)}\left(M(X) \otimes \mathbb{L}_{S}^{r}, M(Y)\right)$ is an element of the form $\psi \circ\left(I d \otimes p_{r}\right)$, with $\psi$ in $C H^{d+r}\left(\left(\mathbb{P}_{S}^{r} \times{ }_{S} X\right) \times_{S} Y, \mathbb{Q}\right)$. The computation in [31, p. 460] shows that the morphism

$$
f^{*}: C H^{d+r}\left(X \times_{S} Y, \mathbb{Q}\right) \rightarrow C H^{d+r}\left(\left(\mathbb{P}_{S}^{r} \times_{S} X\right) \times_{S} Y, \mathbb{Q}\right) \circ\left(p_{r} \otimes I d\right)
$$

is an isomorphism.

Now assume $r=0$. An element of $\operatorname{Hom}_{\mathcal{M}_{+}^{o}(S)}\left(M(X), M(Y) \otimes \mathbb{L}_{S}^{s}\right)$ is an element of the form $\left(I d \otimes p_{s}\right) \circ \psi$, with $\psi$ in $C H^{d}\left(X \times{ }_{S}\left(Y \times \mathbb{P}_{S}^{s}\right), \mathbb{Q}\right)$. By an analogous computation, the morphism

$$
f_{*}:\left(I d \otimes p_{s}\right) \circ C H^{d}\left(X \times_{S}\left(Y \times_{S} \mathbb{P}_{S}^{s}\right), \mathbb{Q}\right) \rightarrow C H^{d-s}\left(X \times_{S} Y, \mathbb{Q}\right)
$$

is an isomorphism.

Definition 6.5 (Chow group of a motive and cup product). For any object $M$ in $\mathcal{M}_{+}^{o}(S)$, and any integer $d \geq 0$, we put

$$
C H^{d}(M, \mathbb{Q}):=\operatorname{Hom}_{\mathcal{M}_{+}^{o}(S)}\left(\mathbb{L}^{d}, M\right)
$$

If $\xi$ is an element of $C H^{d}(M, \mathbb{Q})$, we denote by $\xi \cup($.$) the composition$

$$
M \otimes \mathbb{L}_{S}^{d} \stackrel{I d \otimes \xi}{\longrightarrow} M \otimes M \stackrel{\Delta_{M}}{\longrightarrow} M
$$

where $\Delta_{M}$ is the diagonal morphism (i.e. if $M$ is $(M(Y), p)$ with $Y$ a smooth projective $S$-variety and $p$ a projector on $M(Y)$, then $\left.\Delta_{M}=p \circ M\left(\Delta_{Y}\right) \circ(p \otimes p)\right)$.

Lemma 6.6. For any object $X$ in $\mathcal{V}_{S}$, for any pair of integers $r, s \geq 0$, and for any object $N$ in $\mathcal{M}_{+}^{o}(S)$, there is a canonical isomorphism

$$
H_{\operatorname{som}_{\mathcal{M}_{+}^{o}(S)}}\left(M(X) \otimes \mathbb{L}_{S}^{r}, N \otimes \mathbb{L}_{S}^{s}\right) \cong \oplus_{X_{i} \in \mathcal{C}(X)} C H^{d\left(X_{i} / S\right)+r-s}\left(M\left(X_{i}\right) \otimes N, \mathbb{Q}\right)
$$


where $\mathcal{C}(X)$ denotes the set of connected components of $X$, and $d\left(X_{i} / S\right)$ is the relative dimension of $X_{i}$ over $S$.

Proof. We may suppose that $X$ has pure dimension $d$ over $S$. The result holds if $N=M(Y)$ for some object $Y$ in $\mathcal{V}_{S}$, by Lemma 6.4. In general, $N$ is of the form $(M(Y), p)$, with $p$ a projector on $M(Y)$. We have

$$
\begin{aligned}
C H^{d+r-s}(M(X) \otimes N, \mathbb{Q}) & \cong(I d \otimes p) \circ H_{o m}{\mathcal{M}_{+}^{o}(S)}\left(\mathbb{L}^{d+r-s}, M(X) \otimes M(Y)\right) \\
& \cong(p \otimes I d) \circ H_{\mathcal{M}_{+}^{o}(S)}\left(M(X) \otimes \mathbb{L}_{S}^{r}, M(Y) \otimes \mathbb{L}_{S}^{s}\right) \\
& \cong \operatorname{Hom}_{\mathcal{M}_{+}^{o}(S)}\left(M(X) \otimes \mathbb{L}_{S}^{r}, N \otimes \mathbb{L}_{S}^{s}\right)
\end{aligned}
$$

\subsection{Manin's identity principle.}

Lemma 6.7. We denote by $V e c_{\mathbb{Q}}$ the category of $\mathbb{Q}$-vector spaces. Let $N$ be any object of $\mathcal{M}_{+}^{o}(S)$, and consider the functor

$$
\omega_{N}: \mathcal{V}_{S}^{o p} \rightarrow V e c_{\mathbb{Q}}
$$

defined by $\omega_{N}(Y)=\operatorname{Hom}_{\mathcal{M}_{+}^{o}(S)}(M(Y), N)$. The functor

$$
\omega: \mathcal{M}_{+}^{o}(S) \rightarrow \operatorname{PreSh}\left(\mathcal{V}_{S}\right): N \mapsto \omega_{N}
$$

is fully faithful; here PreSh $\left(\mathcal{V}_{S}\right)$ denotes the category of presheaves of $\mathbb{Q}$-vector spaces on $\mathcal{V}_{S}$.

Proof. By Yoneda's Lemma, the functor

$$
h: \mathcal{M}_{+}^{o}(S) \rightarrow \operatorname{PreSh}\left(\mathcal{M}_{+}^{o}(S)\right): N \mapsto h_{N}:=\operatorname{Hom}_{\mathcal{M}_{+}^{o}(S)}(., N)
$$

is fully faithful. The composition $\omega$ of $h$ with the restriction functor

$$
\operatorname{PreSh}\left(\mathcal{M}_{+}^{o}(S)\right) \rightarrow \operatorname{PreSh}\left(\mathcal{V}_{S}\right)
$$

is still fully faithful, since $h$ is recovered from $\omega$ by putting

$$
h_{(M(Y), p)}(N):=\operatorname{Im}\left\{\omega_{N}(p): \omega_{N}(Y) \rightarrow \omega_{N}(Y)\right\}
$$

Proposition 6.8 (Manin's identity prinicple). Let $f, g: M \rightarrow N$ be morphisms in $\mathcal{M}_{+}^{o}(S)$.

- The morphism $f$ is an isomorphism iff the morphism

$$
\omega(f)(Y): C H^{d}(M(Y) \otimes M, \mathbb{Q}) \rightarrow C H^{d}(M(Y) \otimes N, \mathbb{Q})
$$

is an isomorphism for any integer $d \geq 0$, and any object $Y$ in $\mathcal{V}_{S}$ of pure dimension d over $S$. Moreover, $f=g$ iff $\omega(f)(Y)=\omega(g)(Y)$ for all these pairs $(d, Y)$.

- A sequence

$$
M \stackrel{a}{\longrightarrow} N \stackrel{b}{\longrightarrow} P
$$

in $\mathcal{M}_{+}^{o}(S)$ is split exact, iff a admits a right inverse $a^{\prime}$ and the sequence

$C H^{d}(M(Y) \otimes M, \mathbb{Q}) \stackrel{\omega(a)(Y)}{\longrightarrow} C H^{d}(M(Y) \otimes N, \mathbb{Q}) \stackrel{\omega(b)(Y)}{\longrightarrow} C H^{d}(M(Y) \otimes P, \mathbb{Q})$ is a short exact sequence, for any integer $d \geq 0$, and any object $Y$ in $\mathcal{V}_{S}$ of pure dimension d over $S$.

Proof. This follows from Lemma 6.6] Lemma 6.7 and the proposition in [31, p. 453]. 
6.5. Relative Chow motive of a projective bundle. Let $X$ be a smooth quasiprojective variety over $k$. In this section, we compute the relative Chow motive of a projective bundle $\pi: \mathbb{P}(\mathcal{E}) \rightarrow X$, where $\mathcal{E}$ is a locally free sheaf of modules on $X$ of rank $r+1$. We show that Manin's formula [31, p. 457] in $\mathcal{M}_{+}^{o}(k)$ holds already in $\mathcal{M}_{+}^{o}(X)$. The proof closely follows the arguments in [34, 2.4].

Proposition 6.9. Let $X$ be a smooth quasi-projective variety over $k$, and let $\mathcal{E}$ be a locally free sheaf of modules on $X$ of rank $r+1$. Then

$$
M(\mathbb{P}(\mathcal{E})) \cong \oplus_{i=0}^{r} \mathbb{L}_{X}^{i}
$$

in $\mathcal{M}_{+}^{o}(X)$.

Proof. Consider the tautological bundle $\mathcal{O}_{\mathbb{P}(\mathcal{E})}(1)$ on $\mathbb{P}(\mathcal{E})$, and its divisor class $\xi:=c_{1}\left(\mathcal{O}_{\mathbb{P}(\mathcal{E})}(1)\right)$ in $C H^{1}(\mathbb{P}(\mathcal{E}), \mathbb{Q})$. By Lemma 6.4 the element $\xi^{i} \in C H^{i}(\mathbb{P}(\mathcal{E}))$ corresponds to a morphism $\xi_{*}^{i}: \mathbb{L}_{X}^{i} \rightarrow M(\mathbb{P}(\mathcal{E}))$ in $\mathcal{M}_{+}^{o}(X)$, for any integer $i \geq 0$. We will show that the morphism

$$
\psi: \oplus_{i} \xi_{*}^{i}: \oplus_{i} \mathbb{L}_{X}^{i} \rightarrow M(\mathbb{P}(\mathcal{E}))
$$

is an isomorphism of relative motives over $X$.

By Manin's identity principle in Proposition 6.8 it suffices to show that for any integer $d \geq 0$ and any object $f: Y \rightarrow X$ in $\mathcal{V}_{X}$ of pure relative dimension $d$,

$$
\omega(\psi)(Y): \oplus_{i} C H^{d}\left(M(Y) \otimes \mathbb{L}_{X}^{i}, \mathbb{Q}\right) \rightarrow C H^{d}\left(Y \times_{X} \mathbb{P}(\mathcal{E}), \mathbb{Q}\right)
$$

is an isomorphism. However, $Y \times_{X} \mathbb{P}(\mathcal{E}) \cong \mathbb{P}\left(f^{*} \mathcal{E}\right)$, and $f^{*}(\xi)$ is the divisor class

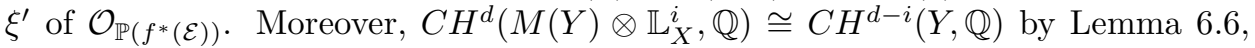
and the morphism $C H^{d-i}(Y, \mathbb{Q}) \rightarrow C H^{d}\left(\mathbb{P}\left(f^{*} \mathcal{E}\right), \mathbb{Q}\right)$ is intersection with $\left(\xi^{\prime}\right)^{i}$. So it follows from Grothendieck's computation of the Chow group of a projective bundle on a smooth quasi-projective variety [26. I.11], that $\omega(\psi)(Y)$ is an isomorphism.

Corollary 6.10. Let $X$ be a smooth projective variety over $S$, and let $\mathcal{E}$ be a locally free sheaf of modules on $X$ of rank $r+1$. Denote by $\xi$ the divisor class $c_{1}\left(\mathcal{O}_{\mathbb{P}(\mathcal{E})}(1)\right)$ of the tautological line bundle on $\mathbb{P}(\mathcal{E})$, and by $h: \mathbb{P}(\mathcal{E}) \rightarrow X$ the projection. Then

$$
\sum_{i=0}^{r} \xi^{i} \cup(h \otimes I d)^{*}: \oplus_{i=0}^{r}\left(M(X) \otimes \mathbb{L}_{S}^{i}\right) \rightarrow M(\mathbb{P}(\mathcal{E}))
$$

is an isomorphism in $\mathcal{M}_{+}^{o}(S)$.

Proof. This is the image of the isomorphism in Proposition 6.9 under the forgetful functor $\mathcal{M}_{+}^{o}(X) \rightarrow \mathcal{M}_{+}^{o}(S)$.

6.6. The blow-up complex. Let $S$ be, as before, a smooth irreducible quasiprojective variety over $k$. Let $X$ be a smooth projective irreducible variety over $S$, and let $Z$ be a closed irreducible subvariety of $X$ of codimension $r$, smooth over $S$. Consider the blow-up $h: X^{\prime} \rightarrow X$ of $X$ at $Z$, and denote by $E$ its exceptional variety. We denote by $i$ and $j$ the inclusions of $Z$ in $X$, resp. $E$ in $X^{\prime}$, and we denote by $h_{E}: E \rightarrow Z$ the restriction of $h$.

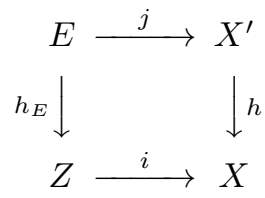

We denote by $N$ the normal bundle of $Z$ in $X$. 
Let $\zeta \in C H^{r-1}(E, \mathbb{Q})$ be the $(r-1)$-th Chern class of the quotient bundle $h^{*}(N) / \mathcal{O}_{E}(-1)$ on $E$. We denote by

$$
i_{*} \in H^{\circ o m} \mathcal{M}_{+}^{o(S)}\left(M(Z) \otimes \mathbb{L}_{S}^{r}, M(X)\right)=C H^{d(Z / S)+r}\left(Z \times_{S} X, \mathbb{Q}\right)
$$

the morphism defined by the transpose of the graph of $i$. The morphism

$$
j_{*}: M(E) \otimes \mathbb{L}_{S} \rightarrow M\left(X^{\prime}\right)
$$

is defined analogously. We'll write $\left(h_{E}\right)_{*}$ for the morphism in

$$
H_{o m} \mathcal{M}_{+}^{o(S)}\left(M(E) \otimes \mathbb{L}_{S}, M(Z) \otimes \mathbb{L}_{S}^{r}\right)=C H^{d(E / S)-(r-1)}\left(E \times_{S} Z, \mathbb{Q}\right)
$$

defined by the transpose of the graph of $h_{E}$. With some abuse of notation, we'll also write $\zeta \cup$ for the morphism $M(E) \otimes \mathbb{L}_{S}^{r} \rightarrow M(E) \otimes \mathbb{L}_{S}$ obtained by twisting $\zeta \cup$ (i.e. tensoring with the identity on $\mathbb{L}_{S}$ ).

Lemma 6.11. Let $\xi$ be the divisor class of the tautological bundle $\mathcal{O}_{E}(1)$ on $E$. The morphism

$$
\varphi: M(X) \oplus\left(\oplus_{i=1}^{r-1} M(Z) \otimes \mathbb{L}_{S}^{i}\right) \rightarrow M\left(X^{\prime}\right)
$$

defined by

$$
\varphi\left(x, y_{1}, \ldots, y_{r-1}\right)=h^{*}(x)+\sum_{i=1}^{r-1} j_{*}\left(\xi^{i-1} \cup\left(h_{E} \otimes I d\right)^{*}\left(y_{i}\right)\right)
$$

is an isomorphism in $\mathcal{M}_{+}^{o}(S)$.

Proof. By Manin's identity principle in Proposition 6.8 it suffices to show that, for any integer $d \geq 0$, and any smooth projective $S$-variety of pure relative dimension $d$,

$$
\omega(\varphi)(Y): C H^{d}\left(Y \times_{S} X, \mathbb{Q}\right) \oplus\left(\oplus_{i=1}^{r-1} C H^{d-i}\left(Y \times_{S} Z, \mathbb{Q}\right)\right) \rightarrow C H^{d}\left(Y \times_{S} X^{\prime}, \mathbb{Q}\right)
$$

is an isomorphism.

Since blow-up commutes with flat base change, $h^{Y}: Y \times_{S} X^{\prime} \rightarrow Y \times_{S} X$ is the blow-up with center $Y \times_{S} Z$ and exceptional divisor $Y \times_{S} E$. Moreover, the divisor class of $\mathcal{O}_{Y \times_{S} E}(1)$ is the pull-back of the divisor class of $\mathcal{O}_{E}(1)$. Hence, it suffices to show that the morphism

$$
\begin{array}{r}
C H^{d}(X, \mathbb{Q}) \oplus\left(\oplus_{i=1}^{r-1} C H^{d-i}(Z, \mathbb{Q})\right) \rightarrow C H^{d}\left(X^{\prime}, \mathbb{Q}\right): \\
\left(x, y_{1}, \ldots, y_{r-1}\right) \mapsto h^{*}(x)+\sum_{i=1}^{r-1} j_{*}\left(\xi^{i-1} \cup h_{E}^{*}\left(y_{i}\right)\right)
\end{array}
$$

is an isomorphism for each $d$. This is done in [4, 0.1.3].

Theorem 6.12 (Blow-up complex). Let $X$ be a smooth projective irreducible variety over $S$, and let $Z$ be a closed irreducible subvariety of $X$ of codimension $r$, smooth over $S$. Consider the blow-up $h: X^{\prime} \rightarrow X$ of $X$ at $Z$, and denote by $E$ its exceptional variety. We denote by $i$ and $j$ the inclusions of $Z$ in $X$, resp. $E$ in $X^{\prime}$, and we denote by $h_{E}: E \rightarrow Z$ the restriction of $h$. The complex

$$
0 \longrightarrow M(X) \stackrel{h^{*}+i^{*}}{\longrightarrow} M\left(X^{\prime}\right) \oplus M(Z) \stackrel{j^{*}-h_{E}^{*}}{\longrightarrow} M(E) \longrightarrow 0
$$

splits. In particular,

$$
[M(X)]+[M(E)]=\left[M\left(X^{\prime}\right)\right]+[M(Z)]
$$

in $K_{0}\left(\mathcal{M}_{+}^{o}(S)\right)$. 
If $G$ is a finite group, $X$ carries a good $G$-action, and $Z$ is $G$-closed, then the splitting is $G$-equivariant.

Proof. The absolute case $S=\operatorname{Spec} k$ was proven in [27, 5.1].

Let $\xi$ be the divisor class of the tautological bundle $\mathcal{O}_{E}(1)$ on $E$. We've seen in Corollary 6.10 that

$$
\psi\left(y_{0}, \ldots, y_{r}\right)=\sum_{i=0}^{r-1} \xi^{i} \cup\left(h_{E} \otimes I d\right)^{*}\left(y_{i}\right)
$$

defines an isomorphism

$$
\psi: \oplus_{i=0}^{r-1} M(Z) \otimes \mathbb{L}_{S}^{i} \rightarrow M(E)
$$

in $\mathcal{M}_{+}^{o}(S)$.Combining this expression with the isomorphism $\varphi$ in Lemma 6.11 and putting $A:=\oplus_{i=1}^{r-1} M(Z) \otimes \mathbb{L}_{S}^{i}$, we get an isomorphism of complexes

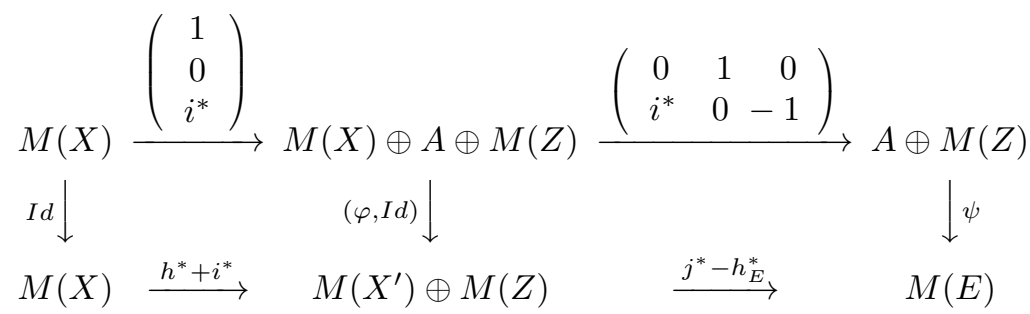

Commutativity of the squares follows from the equality $j^{*} j_{*}=\xi \cup$ (see the proof of [21, 6.7(c)]). It is clear that the upper complex splits.

If $X$ carries a good $G$-action, and $Z$ is $G$-closed, then all the morphisms used in the proof are $G$-equivariant, and the splitting is $G$-equivariant.

Remark. In the classical setting, where $k=\mathbb{C}, S=$ Spec $\mathbb{C}$, and where we use singular homology instead of motives, the exact blow-up sequence arises as follows [25. p.605]: take tubular neighbourhoods $U$ and $V$ of $Z$ and $E$ in $X$, resp. $X^{\prime}$. We have $H_{*}(U)=H_{*}(Z), H_{*}(V)=H_{*}(E), H_{*}(U-Y)=H_{*}(V-E)$, and $H_{*}\left(X^{\prime}-E\right)=$ $H_{*}(X-Z)$. Hence, combining the Mayer-Vietoris sequences for $X=U \cup(X-Z)$ and $X^{\prime}=V \cup\left(X^{\prime}-E\right)$, with the Barrat-Whitehead Lemma [24, 17.4], and the fact that $H_{*}\left(X^{\prime}\right) \rightarrow H_{*}(X)$ is surjective, we obtain a short exact sequence

$$
0 \rightarrow H_{*}(E) \rightarrow H_{*}(Z) \oplus H_{*}\left(X^{\prime}\right) \rightarrow H_{*}(X) \rightarrow 0
$$

6.7. Direct limits of categories. Let $I$ be a directed set, and let $\left(\mathcal{C}_{i}\right)_{i \in I}$ be a direct system of categories, with transition functors $F_{j, i}$ for $j \geq i$ in $I$.

Definition 6.13. We define the direct limit $\mathcal{C}$ of $\left(\mathcal{C}_{i}\right)_{i \in I}$ as follows:

- Put $O:=\sqcup_{i \in I} O b\left(\mathcal{C}_{i}\right)$. On this class, we define an equivalence relation $\sim$ as follows: for any two objects $(i, \alpha)$ and $(j, \beta)$, with $\alpha, \beta$ objects of $\mathcal{C}_{i}$, resp. $\mathcal{C}_{j},(i, \alpha) \sim(j, \beta)$ iff there exists an index $k \geq i, j$ in $I$ such that $F_{k, i}(\alpha)=F_{k, j}(\beta)$. We put $O b(\mathcal{C}):=O / \sim$. 
- Consider two objects $A$ and $B$ in $O b(\mathcal{C})$, represented by $(i, \alpha)$, resp. $(j, \beta)$ in $O$. Put

$H((i, \alpha),(j, \beta)):=\left\{(k, f) \mid k \in I\right.$, with $k \geq i, j$, and $\left.f \in \operatorname{Hom}_{\mathcal{C}_{k}}\left(F_{k, i}(\alpha), F_{k, j}(\beta)\right)\right\}$

On this class, we define an equivalence relation $\sim$ as follows: $(k, f) \sim$ $\left(k^{\prime}, f^{\prime}\right)$ iff there exists an element $\ell$ in $I$, with $\ell \geq k$ and $\ell \geq k^{\prime}$, such that $F_{\ell, k}(f)=F_{\ell, k^{\prime}}\left(f^{\prime}\right)$. Observe that this equivalence relation is compatible with the composition of morphisms. We put

$$
\operatorname{Hom}_{\mathcal{C}}(A, B):=H((i, \alpha),(j, \beta)) / \sim
$$

This definition does not depend on the choice of the objects $(i, \alpha)$ and $(j, \beta)$ in $O$ representing $A$ and $B$.

For each $i \in I$, there is a natural functor $F_{i}: \mathcal{C}_{i} \rightarrow \mathcal{C}$, mapping an object $\alpha$ to the class of $(i, \alpha)$, and mapping a morphism $f$ to $(i, f)$.

If all the $\mathcal{C}_{i}$ are small categories, then $\mathcal{C}$ is a direct limit in the category of small categories. In any case, it has the following universal property: $F_{i}=F_{j} \circ F_{j, i}$ for any pair $j \geq i$ in $I$, and if $\mathcal{D}$ is any category, and $G_{i}: \mathcal{C}_{i} \rightarrow \mathcal{D}$ is a system of functors such that $G_{i}=G_{j} \circ F_{j, i}$ for any pair $j \geq i$ in $I$, then there exists a unique functor $G: \mathcal{C} \rightarrow \mathcal{D}$ satisfying $G_{i}=G \circ F_{i}$ for any $i \in I$.

If the categories $\mathcal{C}_{i}$ are $\mathbb{Q}$-linear with tensor structure, and if the transition functors respect $\mathbb{Q}$-linearity and tensor structure, then $\mathcal{C}$ is a $\mathbb{Q}$-linear category with tensor structure in a natural way, and the natural morphisms $F_{i}$ respect $\mathbb{Q}$ linearity and tensor structure.

If $\left(\mathcal{C}_{i}\right)_{i \in I}$ is a direct system of pseudo-abelian categories $\mathcal{C}_{i}$, with additive transition functors, then $\mathcal{C}$ is pseudo-abelian, since additive functors preserve direct sums.

6.8. Constructible Chow motives. Now, let $S$ be any $k$-variety. An admissible stratification is a finite stratification $\mathscr{S}=\left\{S_{1}, \ldots, S_{p}\right\}$ of $S$ into smooth, irreducible, quasi-projective, locally closed subvarieties $S_{i}$. The admissible stratifications of $S$ form a directed set (where $\mathscr{S} \leq \mathscr{S}^{\prime}$ iff $\mathscr{S}^{\prime}$ is a refinement of $\mathscr{S}$ ). We define $\mathcal{M}_{+}^{o}(S, \mathscr{S})$ as the direct product of categories $\prod_{i=1}^{p} \mathcal{M}_{+}^{o}\left(S_{i}\right)$. Base change induces restriction functors

$$
F_{\mathscr{S}^{\prime}, \mathscr{S}}: \mathcal{M}_{+}^{o}(S, \mathscr{S}) \rightarrow \mathcal{M}_{+}^{o}\left(S, \mathscr{S}^{\prime}\right)
$$

where $\mathscr{S}^{\prime}$ is an admissible refinement of $\mathscr{S}$. We obtain an inductive system of $\mathbb{Q}$-linear categories with tensor structure, indexed by the admissible stratifications $\mathscr{S}$ of $S$.

Definition 6.14. We define the category $C M$ ot $_{S}$ of constructible Chow motives over $S$, as the direct limit of the direct system $\left(\mathcal{M}_{+}^{o}(S, \mathscr{S})\right)_{\mathscr{S}}$. The category $C_{\text {Mot }}$ is $\mathbb{Q}$-linear, with tensor structure, and pseudo-abelian. There are natural $\mathbb{Q}$-linear tensor functors

$$
F_{\mathscr{S}}: \mathcal{M}_{+}^{o}(S, \mathscr{S}) \rightarrow \mathrm{CMot}_{S}
$$

By Definition [6.1, we can associate a Grothendieck ring $K_{0}\left(C M o t_{S}\right)$ to the additive tensor category $\mathrm{CMot}_{S}$.

Lemma 6.15.

$$
K_{0}\left(\mathrm{CMot}_{S}\right) \cong \lim _{\overrightarrow{\mathscr{S}}} \prod_{S_{i} \in \mathscr{S}} K_{0}\left(\mathcal{M}_{+}^{o}\left(S_{i}\right)\right)
$$


where $\mathscr{S}$ runs over the admissible stratifications of $S$.

Proof. The canonical functors $F_{\mathscr{S}}$ induce ring morphisms

$$
K_{0}\left(F_{\mathscr{S}}\right): \prod_{S_{i} \in \mathscr{S}} K_{0}\left(\mathcal{M}_{+}^{o}\left(S_{i}\right)\right) \cong K_{0}\left(\prod_{S_{i} \in \mathscr{S}} \mathcal{M}_{+}^{o}\left(S_{i}\right)\right) \rightarrow K_{0}\left(C M o t_{S}\right)
$$

and hence a ring morphism

$$
\psi: \lim _{\overrightarrow{\mathscr{S}}} \prod_{S_{i} \in \mathscr{S}} K_{0}\left(\mathcal{M}_{+}^{o}\left(S_{i}\right)\right) \rightarrow K_{0}\left(C M o t_{S}\right)
$$

It is clear that $\psi$ is surjective, so let us prove injectivity. Let $\mathscr{S}$ be an admissible stratification of $S$. Let $\alpha$ be an element of $K_{0}\left(\mathcal{M}_{+}^{o}(S, \mathscr{S})\right)$, and suppose that $\alpha$ maps to zero under $K_{0}\left(F_{\mathscr{S}}\right)$. This means that there exist objects $A$ and $B$ in $\mathcal{M}_{+}^{o}(S, \mathscr{S})$, and an object $C$ in $C M o t_{S}$, such that $\alpha+[B]=[A]$, and $F_{\mathscr{S}}(A) \oplus C$ and $F_{\mathscr{S}}(B) \oplus C$ are isomorphic. By definition of the direct limit $C M$ ot $_{S}$, this implies that there exists a refinement $\mathscr{S}^{\prime}$ of $\mathscr{S}$, and an object $\mathcal{C}^{\prime}$ in $\mathcal{M}_{+}^{o}\left(\mathscr{S}^{\prime}\right)$, such that $F_{\mathscr{S}^{\prime}, \mathscr{S}}(A) \oplus C$ and $F_{\mathscr{S}^{\prime}, \mathscr{S}}(B) \oplus C$ are isomorphic. Hence, $\alpha$ maps to zero in

$$
\lim _{\overrightarrow{\mathscr{S}}} \prod_{S_{i} \in \mathscr{S}} K_{0}\left(\mathcal{M}_{+}^{o}\left(S_{i}\right)\right)
$$

Corollary 6.16. The realization functors $\mathcal{M}_{+}^{o}(S) \rightarrow D^{b}\left(S, \mathbb{Q}_{\ell}\right)$ for smooth quasiprojective k-varieties $S$, induce a realization morphism of Grothendieck rings

$$
K_{0}\left(C M o t_{S}\right) \rightarrow K_{0}\left(D^{b}\left(S, \mathbb{Q}_{\ell}\right)\right)
$$

for any $k$-variety $S$. Here we take the Grothendieck ring of $D^{b}\left(S, \mathbb{Q}_{\ell}\right)$ as a triangulated category, i.e. if $A \rightarrow B \rightarrow C \rightarrow A[1]$ is a distinguished triangle in $D^{b}\left(S, \mathbb{Q}_{\ell}\right)$, then $[B]=[A]+[C]$ in $K_{0}\left(D^{b}\left(S, \mathbb{Q}_{\ell}\right)\right)$.

Proof. We define, for any admissible stratification $\mathscr{S}$ of $S$.

$$
\psi_{\mathscr{S}}: K_{0}\left(\mathcal{M}_{+}^{o}(S, \mathscr{S})\right) \rightarrow K_{0}\left(D^{b}\left(S, \mathbb{Q}_{\ell}\right)\right)
$$

as follows: if $S_{i}$ is a stratum of $\mathscr{S}$, and $j: S_{i} \rightarrow S$ is the inclusion, then

$$
K_{0}\left(\mathcal{M}_{+}^{o}\left(S_{i}\right)\right) \rightarrow K_{0}\left(D^{b}\left(S, \mathbb{Q}_{\ell}\right)\right)
$$

is the composition of

$$
K_{0}(\text { real }): K_{0}\left(\mathcal{M}_{+}^{o}\left(S_{i}\right)\right) \rightarrow K_{0}\left(D^{b}\left(S_{i}, \mathbb{Q}_{\ell}\right)\right)
$$

with

$$
K_{0}\left(j_{!}\right): K_{0}\left(D^{b}\left(S_{i}, \mathbb{Q}_{\ell}\right)\right) \rightarrow K_{0}\left(D^{b}\left(S, \mathbb{Q}_{\ell}\right)\right)
$$

These morphisms $\psi_{\mathscr{S}}$ form a direct system and pass to a limit morphism

$$
K_{0}\left(C M \operatorname{Mot}_{S}\right) \rightarrow K_{0}\left(D^{b}\left(S, \mathbb{Q}_{\ell}\right)\right)
$$

since for any closed subvariety $V$ of $S$, and any bounded complex of $\mathbb{Q}_{\ell}$-sheaves $\mathcal{F}$ on $S$, we get a distinguished triangle

$$
u ! u^{*} \mathcal{F} \rightarrow \mathcal{F} \rightarrow v_{*} v^{*} \mathcal{F} \rightarrow u_{!} u^{*} \mathcal{F}[1]
$$

in $D^{b}\left(S, \mathbb{Q}_{\ell}\right)$, where $U$ denotes the complement of $V$ in $S$, and $u$ and $v$ are the inclusions of $U$, resp. $V$ in $S$. 
If $f: S^{\prime} \rightarrow S$ is a morphism of smooth, quasi-projective $k$-varieties, then the base-change functor $\mathcal{M}_{+}^{o}(S) \rightarrow \mathcal{M}_{+}^{o}\left(S^{\prime}\right)$ induces a base-change functor $\operatorname{CMot}_{S} \rightarrow$ $C M$ ot $_{S^{\prime}}$. If $S^{\prime}$ is a smooth projective $S$-variety, the forgetful functor from Lemma 6.2 will in general not pass to a forgetful functor on constructible Chow motives (due to the stratifications we allowed on the base).

6.9. Extension of cohomological functors. Let $G$ be a fixed finite group. Let $S$ be a variety over a field $k$ of characteristic zero.

Theorem 2.2.2 in [27] gives a very useful criterion to extend cohomological functors defined on smooth and projective varieties over $k$. We will extend this result to the relative case, working over the base $S$.

Their cohomological theories take values in so-called cohomological descent categories [27, 1.7.1]. These are tuples $(D, E, s, \lambda, \mu)$ which capture the essential properties of the category of complexes over an abelian category, and the class of quasiisomorphisms. For our purposes, it suffices to recall that one can associate a cohomological descent category to any additive category $\mathcal{A}$, with underlying category $D=\mathcal{C}^{b}(\mathcal{A})$, where $s$ is the functor that associates to a codiagram of complexes its total complex in $\mathcal{C}^{b}(\mathcal{A})$, and where $E$ is the class of homotopy equivalences, by [27 1.7.7]. For any descent category $(D, E, s, \lambda, \mu)$, we denote by $H o(D)$ the localization of $D$ w.r.t. the class of morphisms $E$.

We denote by $\left(G, \operatorname{Var}_{S}\right)$ the category of varieties over $S$ with good $G$-action. Let $\left(G, \mathcal{V}_{S}\right)$ be the full subcategory of $\left(G, \operatorname{Var}_{S}\right)$ whose objects are the smooth and projective varieties over $S$. We will also consider the category $\left(G, \operatorname{Var}_{S, c}\right)$ of $S$-varieties with good $G$-action, with proper morphisms. If $\left(G, \mathcal{C}_{S}\right)$ is any of these categories, we define $\left(G, \overline{\mathcal{C}}_{S}\right)$ as the inductive limit of $\left(G, \mathcal{C}_{\mathscr{S}}\right):=\prod_{S_{i} \in \mathscr{S}}\left(G, \mathcal{C}_{S_{i}}\right)$ over the admissible stratifications $\mathscr{S}=\left\{S_{i}\right\}_{i}$ of $S$ (see Section 6.8). Beware: if $S_{i}$ is a locally closed subvariety of $S$, and $X$ is an $S$-variety, the restriction of $X$ to $S_{i}$ is given by $\left(X \times{ }_{S} S_{i}\right)_{\text {red }}$ with its reduced structure.

Definition 6.17 (Acyclic diagrams). An acyclic diagram in $\left(G, \operatorname{Var}_{\mathscr{S}}\right)$, for some admissible stratification $\mathscr{S}=\left\{S_{i}\right\}$ of $S$, is a set of $G$-equivariant Cartesian diagrams of the type

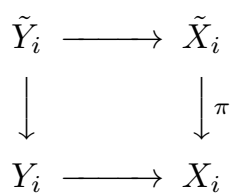

where $X_{i}, Y_{i}$ are $S_{i}$-varieties with good $G$-action, the horizontal arrows are closed immersions, the vertical arrows are proper $S_{i}$-morphisms, and $\pi$ induces an isomorphism $\tilde{X}_{i} \backslash \tilde{Y}_{i} \cong X_{i} \backslash Y_{i}$.

An acyclic diagram in $\overline{\operatorname{Var}}_{S}$ is the image of an acyclic diagram in $\left(G, \operatorname{Var}_{\mathscr{S}}\right)$ under the natural functor

$$
F_{\mathscr{S}}:\left(G, \operatorname{Var}_{\mathscr{S}}\right) \rightarrow\left(G, \overline{\operatorname{Var}}_{S}\right)
$$

for some admissible stratification $\mathscr{S}$ of $S$.

An acyclic diagram in $\left(G, \overline{V a r}_{S, c}\right)$ is a diagram in $\left(G, \overline{V a r}_{S, c}\right)$ which is acyclic in $\left(G, \overline{\operatorname{Var}}_{S}\right)$. 
An elementary acyclic diagram in $\left(G, \overline{\mathcal{V}}_{S}\right)$ is a blow-up diagram in $\left(G, \overline{\mathcal{V}}_{S}\right)$, i.e. an acyclic diagram in $\left(G, \overline{\operatorname{Var}}_{S}\right)$ where all objects belong to $\left(G, \overline{\mathcal{V}}_{S}\right)$, and $\pi$ is the blow-up of $X_{i}$ at the $G$-closed center $Y_{i}$.

An (elementary) acyclic morphism in any of the above categories, is a morphism that can be realized as the right vertical arrow in an (elementary) acyclic diagram.

The following Theorem is a relative version of [27], Theorem 2.2.2 and [10], Theorem 3.1. Let $(D, E, s, \mu, \lambda)$ be a cohomological descent category.

Theorem 6.18 (Extension Theorem). Given a contravariant functor $F$ from $\left(G, \overline{\mathcal{V}}_{S}\right)$ to a cohomological descent category $(D, E, s, \mu, \lambda)$ such that

(F1) $F(\emptyset)=0$,

(F2) the natural morphism $F(X \sqcup Y) \rightarrow F(X) \times F(Y)$ is an isomorphism,

(F3) for any elementary acyclic diagram $X_{\bullet}$, the object $s F\left(X_{\bullet}\right)$ is acyclic in $H o(D)$,

there exists an essentially unique extension of $F$ to a functor

$$
F_{c}:\left(G, \overline{\operatorname{Var}}_{S, c}\right) \rightarrow H o(D)
$$

such that the following descent properties hold:

(D) for any acyclic diagram $X_{\bullet}$ in $\left(G, \overline{\operatorname{Var}}_{S, c}\right)$, the object $s F\left(X_{\bullet}\right)$ is acyclic.

(E) for any $S$-variety $X$ with good $G$-action, and any $G$-closed, closed subvariety $Y$, we have a natural isomorphism

$$
F_{c}(X \backslash Y) \cong s\left(F_{c}(Y \hookrightarrow X)\right)
$$

Moreover, if $H: \overline{\mathcal{V}}_{S} \rightarrow D$ is another functor satisfying $\left(F_{1}\right),\left(F_{2}\right)$ and $\left(F_{3}\right), H^{\prime}$ is an extension of $H$ satisfying $(D)$ and $(E)$, and $\tau: F \rightarrow H$ is any natural transformation, then $\tau$ extends uniquely to a natural transformation $\tau: F^{\prime} \rightarrow H^{\prime}$. This extension $\tau^{\prime}$ is an isomorphism if $\tau$ is.

Proof. As explained in 10], Proof of Theorem 2.2, and in the appendix of [1], it suffices to use Proposition 5.4 Proposition 5.5] and Proposition [5.6 combined with the fact that all the objects in $\overline{\operatorname{Var}}_{S, c}$ of dimension zero are in $\overline{\mathcal{V}}_{S}$ (we de not claim that any variety of relative dimension 0 over $S$ is smooth over $S$, but merely that this is true after admissible stratification of the base $S$ ).

Now one can use the methods from [27] (in particular, the theory of cubical hyperresolutions) to prove Theorem 6.18

6.10. Associating a constructible motive to a family of varieties with group action. Let $G$ be a fixed finite group. Let $S$ be a variety over a field $k$ of characteristic zero.

For any additive category $\mathcal{A}$, we denote by $(G, \mathcal{A})$ the additive category of functors $G \rightarrow \mathcal{A}$, where we view $G$ as a category with one object, and automorphism group $G$. We denote by $H o\left(G, \mathcal{C}^{b}(\mathcal{A})\right)=H_{o} \mathcal{C}^{b}(G, \mathcal{A})$ the homotopy category associated to $(G, \mathcal{A})$, i.e. the category of bounded complexes over $(G, \mathcal{A})$ localized w.r.t. homotopy equivalences.

If $S$ is a smooth, quasi-projective variety over $k$, we can define a contravariant functor

$$
M_{G}:\left(G, \mathcal{V}_{S}\right) \rightarrow\left(G, \mathcal{M}_{+}^{o}(S)\right)
$$

mapping a smooth and projective $S$-variety $X$ with good $G$-action to its motive $M(X)$, that we endow with a left $G$-action as follows: an element $g$ of $G$ acts by its graph $[g]$ in $A_{u t_{\mathcal{M}_{+}(S)}}(M(X))$. 
For an arbitrary $k$-variety $S$, and any admissible stratification $\mathscr{S}$ of $S$, we have a canonical functor

$$
M_{G}:\left(G, \mathcal{V}_{\mathscr{S}}\right) \rightarrow\left(G, C M o t_{S}\right)
$$

induced by the functors $M: \mathcal{V}_{S_{i}} \rightarrow \mathcal{M}_{+}^{o}\left(S_{i}\right)$ for $S_{i} \in \mathscr{S}$. These induce a canonical functor

$$
M_{G}:\left(G, \overline{\mathcal{V}}_{S}\right) \rightarrow\left(G, C M o t_{S}\right)
$$

Theorem 6.19. The functor

$$
M_{G}:\left(G, \overline{\mathcal{V}}_{S}\right) \rightarrow\left(G, C \operatorname{Mot}_{S}\right)
$$

has an essentially unique extension to a functor

$$
M_{G, c}:\left(G, \overline{\operatorname{Var}}_{S, c}\right) \rightarrow H o\left(G, \mathcal{C}^{b}\left(C M o t_{S}\right)\right)
$$

satisfying properties $(D)$ and $(E)$ in Theorem 6.18 .

Proof. We only have to check properties $(F 1),(F 2),(F 3)$ in Theorem 6.18 While $(F 1)$ and $(F 2)$ are obvious, $(F 3)$ follows from Theorem 6.12

Corollary 6.20. There exists a functor

$$
M_{G, c}:\left(G, \operatorname{Var}_{S, c}\right) \rightarrow H o\left(G, \mathcal{C}^{b}\left(\operatorname{CMot}_{S}\right)\right)
$$

that extends the functor $M_{G}:\left(G, \mathcal{V}_{S}\right) \rightarrow\left(G, \mathcal{M}_{+}^{o}(S)\right)$ and satisfies properties $(D)$ and $(E)$.

In particular, there exists a unique morphism of Grothendieck rings

$$
\chi_{G, c}: K_{0}^{G}\left(\operatorname{Var}_{S}\right) \rightarrow K_{0}\left(G, C M o t_{S}\right)
$$

mapping the class of an object $X$ of $\mathcal{V}_{T}$, with $T$ a smooth, irreducible, quasiprojective locally closed subset of $S$, to the class of $M_{G}(X)$ in $K_{0}\left(G, C M o t_{S}\right)$.

Proof. Use the canonical functor $F_{\{S\}}:\left(G, \operatorname{Var}_{S, c}\right) \rightarrow\left(G, \overline{\operatorname{Var}}_{S, c}\right)$.

The existence of the morphism of Grothendieck rings $\chi_{c, G}$ follows from the application $\mathrm{ObHo}\left(\mathcal{C}^{b}(\mathcal{A})\right) \rightarrow K_{0}(\mathcal{A})$ constructed in [27. 5.4] for any pseudo-abelian category $\mathcal{A}$.

Corollary 6.21. There exists a functor

$$
M_{c}: \operatorname{Var}_{S, c} \rightarrow H o\left(\mathcal{C}^{b}\left(\operatorname{CMot}_{S}\right)\right)
$$

that extends the functor $M: \mathcal{V}_{S} \rightarrow \mathcal{M}_{+}^{o}(S)$ and satisfies properties $(D)$ and $(E)$ (for $G=\{e\}$ ).

In particular, there exists a unique morphism of Grothendieck rings

$$
\chi_{c}: K_{0}\left(\operatorname{Var}_{S}\right) \rightarrow K_{0}\left(\operatorname{CMot}_{S}\right)
$$

mapping the class of a smooth, projective $T$-variety $X$, with $T$ a smooth, irreducible, quasi-projective locally closed subset of $S$, to the class of $M(X)$ in $K_{0}\left(C M o t_{S}\right)$.

Remark. In the Appendix to [5], Bittner used the Weak Factorization Theorem 2] to prove that $K_{0}^{G}\left(\operatorname{Var}_{S}\right)$ can be represented as follows: the set of generators consists of the isomorphism classes [X] of $S$-varieties $X$ with good $G$-action, which are projective and smooth over their image in $S$, and such that $G$ acts transitively on the connected components of $X$. These generators are subject to the following relations:

$$
\text { - }[\emptyset]=0,
$$


- $\left[B l_{Y} X\right]-[E]=[X]-[Y]$, where $Y$ is a closed $G$-invariant subvariety of $X$, smooth over its image in $S$, which coincides with the image of $\mathrm{X}$ in $S$; and $B l_{Y} X$ is the blow-up of $X$ along $Y$, with exceptional divisor $E$,

- $[X]=\left[X_{T}\right]+\left[X_{S \backslash T}\right]$, where $T$ is a closed subvariety of $S$.

The existence and uniqueness of $\chi_{G, c}$ follow immediately from this presentation.

Proposition 6.22. The functor $M_{G, c}$ commutes with base change, i.e. if $S^{\prime} \rightarrow S$ is a morphism of smooth quasi-projective varieties, the diagram

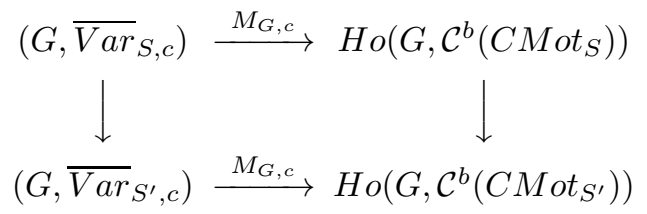

commutes (up to natural isomorphism). In particular, the diagram

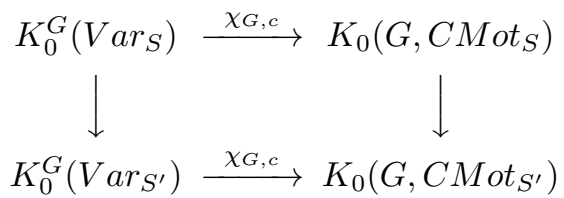

commutes.

Proof. It is clear that both compositions coincide on $\left(G, \overline{\mathcal{V}}_{S}\right)$. Moreover, the base change functor $\left(G, \operatorname{Var}_{S, c}\right) \rightarrow\left(G, \operatorname{Var}_{S^{\prime}, c}\right)$ respects (elementary) acyclic diagrams. So the diagram commutes by the uniqueness result in Theorem 6.18

\section{Character Decomposition of COnStructible motives}

Throughout this section, our base scheme $S$ is a $k$-variety, with $k$ a field of characteristic zero. We will generalize some results from [10] (where $S=\operatorname{Spec} k$ ) to the relative, constructible setting, and we will closely follow their arguments.

For any finite group $G$, and any $S$-variety $X$, we will denote by $G \times X$ the constant finite group $S$-scheme associated to $G$ and $X$. This means that $G \times X:=\sqcup_{g \in G} X$, and $G$ acts on the left and on the right by permuting the indices. We denote by $\mathbf{1}[G]$ the motive $M(G \times S)$ in $C M o t_{S}$. The left and right action of $G$ on $\mathbf{1}[G]$ are called the left, resp. right regular representation of $G$. The object $\mathbf{1}[G]$ is the motivic counterpart of the object $\mathbb{Q}[G]$ in the representation theory of $G$ over $\mathbb{Q}$.

If $M$ is an object of $C M o t_{S}$ and $N$ is an object of $\left(G, C M o t_{S}\right)$, then we view $H_{\text {om }_{C M}}(M, N)$ as a left $\mathbb{Q}[G]$-module, by $(g, f) \mapsto g \circ f$.

7.1. Restriction and induction. Let $\psi: G \rightarrow G^{\prime}$ be a morphism of finite groups. There are obvious restriction functors, both denoted by $R e s_{\psi}$,

$$
\begin{aligned}
\left(G^{\prime}, \overline{\operatorname{Var}}_{S, c}\right) & \rightarrow\left(G, \overline{\operatorname{Var}}_{S, c}\right) \\
H o\left(G^{\prime}, \mathcal{C}^{b}\left(C \operatorname{Mot}_{S}\right)\right) & \rightarrow H o\left(G, \mathcal{C}^{b}\left(\operatorname{CMot}_{S}\right)\right)
\end{aligned}
$$


Proposition 7.1. The square

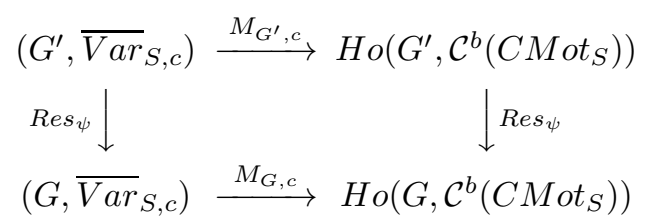

commutes (up to natural isomorphism).

Proof. For objects in $\left(G^{\prime}, \overline{\mathcal{V}}_{S}\right)$, this is clear. By Theorem 6.18 the extension of

$$
M_{G} \circ \operatorname{Res}_{\psi} \cong \operatorname{Res}_{\psi} \circ M_{G^{\prime}}:\left(G^{\prime}, \overline{\mathcal{V}}_{S}\right) \rightarrow\left(G, \mathcal{C}^{b}\left(C M o t_{S}\right)\right)
$$

to $\left(G^{\prime}, \overline{\operatorname{Var}}_{S, c}\right)$ satisfying $(D)$ and $(E)$ is essentially unique. Hence, $M_{G, c} \circ R e s_{\psi} \cong$ $\operatorname{Res}_{\psi} \circ M_{G^{\prime}, c}$.

Lemma 7.2. If $M$ is an object of $\left(G^{\prime}, C M o t_{S}\right)$, and $N$ is an object of $C M o t_{S}$, then $\operatorname{Hom}_{C M o t}(N, M)$ is a left $\mathbb{Q}\left[G^{\prime}\right]$-module, and $\operatorname{Hom}_{C M o t}\left(N, \operatorname{Res}_{\psi} M\right)$ is the usual restriction $\mathrm{Res}_{\psi} \mathrm{Hom}_{C M o t}(N, M)$.

Proof. This is obvious.

We construct a left adjoint $I n d_{\psi}$ for $\operatorname{Res}_{\psi}$ as in [10, Section 4.

If $X$ is an $S$-variety with good $G$-action, then $G$ acts on $G^{\prime} \times X$ by $g\left(g^{\prime}, x\right):=$ $\left(g^{\prime} \psi\left(g^{-1}\right), g x\right)$, and $G^{\prime}$ acts on $G^{\prime} \times X$ by $g\left(g^{\prime}, x\right):=\left(g g^{\prime}, x\right)$. These actions commute, and we put $\operatorname{Ind}_{\psi} X:=\left(G^{\prime} \times X\right) / G$ with its natural $G^{\prime}$-action. This construction commutes with stratification of the base, and we obtain a functor

$$
\operatorname{Ind}_{\psi}:\left(G, \overline{\operatorname{Var}}_{S, c}\right) \rightarrow\left(G^{\prime}, \overline{\operatorname{Var}}_{S, c}\right)
$$

For any $\mathbb{Q}$-linear pseudo-abelian category $\mathcal{A}$, and any object $M$ of $(G, \mathcal{A})$, we can consider the projector $\frac{1}{|G|} \sum_{g \in G}[g]$ on $M$ (where $[g]$ denotes the image of $g$ in the endomorphism class of $M$ ). Its image is denoted by $M^{G}$, and is called the $G$-invariant part of $M$. If $N$ is an object of $\mathcal{A}$, then $G$ acts on the $\mathbb{Q}$-vector space $\operatorname{Hom}_{\mathcal{A}}(N, M)$, and $\operatorname{Hom}_{\mathcal{A}}\left(N, M^{G}\right)$ coincides with the subspace of $G$-invariants $\left(\operatorname{Hom}_{\mathcal{A}}(N, M)\right)^{G}$. We obtain an additive functor $(.)^{G}:(G, \mathcal{A}) \rightarrow \mathcal{A}$.

If $M$ is an object of $\left(G, C M o t_{S}\right)$, we let $G$ act on $\mathbf{1}\left[G^{\prime}\right] \otimes M$ via the inverse of the right regular representation of $G^{\prime}$ and the $G$-action on $M$, and we let $G^{\prime}$ act on $\mathbf{1}\left[G^{\prime}\right] \otimes M$ via the left regular representation of $G^{\prime}$ and the trivial action on $M$. These action commute, and we obtain an object $\operatorname{Ind}_{\psi} M:=\left(\mathbf{1}\left[G^{\prime}\right] \otimes M\right)^{G}$ of $\left(G^{\prime}, C M o t_{S}\right)$. We obtain additive functors, all denoted by $I n d_{\psi}$,

$$
\begin{aligned}
\left(G, C M \mathrm{ot}_{S}\right) & \rightarrow\left(G^{\prime}, \mathrm{CMot}_{S}\right) \\
\left(G, \mathcal{C}^{b}\left(\mathrm{CMot}_{S}\right)\right) & \rightarrow\left(G^{\prime}, \mathcal{C}^{b}\left(\mathrm{CMot}_{S}\right)\right) \\
H o\left(G, \mathcal{C}^{b}\left(\mathrm{CMot}_{S}\right)\right) & \rightarrow H o\left(G^{\prime}, \mathcal{C}^{b}\left(\text { CMot }_{S}\right)\right)
\end{aligned}
$$

If $H$ is a normal subgroup of $G$, and $\psi: G \rightarrow G / H$ is the projection, then $\operatorname{Ind}_{\psi} M$ is $M^{H}$ with its residual $G / H$-action, and for any object $X$ in $\left(G, \overline{\operatorname{Var}}_{S}\right)$, $\operatorname{Ind}_{\psi} X$ is $X / H$. In particular, for $\psi: G \rightarrow\{e\}$, we obtain $M^{G}$ and $X / G$.

Lemma 7.3. If $M$ is an object of $\left(G, C M o t_{S}\right)$, and $N$ is an object of $C M o t_{S}$, then $\operatorname{Hom}_{C M o t}(N, M)$ is a left $\mathbb{Q}[G]$-module, and $\operatorname{Hom}_{C M o t}\left(N, \operatorname{Ind}_{\psi} M\right)$ is the usual induced $\mathbb{Q}\left[G^{\prime}\right]$-module $\operatorname{Ind}_{\psi} \operatorname{Hom}_{\mathrm{CMot}_{S}}(N, M)$. 
Proof. We see that

$$
\operatorname{Hom}_{C M o t}\left(N, \mathbf{1}\left[G^{\prime}\right] \otimes M\right) \cong \mathbb{Q}\left[G^{\prime}\right] \otimes_{\mathbb{Q}} \operatorname{Hom}_{C \operatorname{Mot}_{S}}(N, M)
$$

as a left $\mathbb{Q}\left[G^{\prime}\right]$-module. Hence, it suffices to observe that, for any left $\mathbb{Q}[G]$-module $V$,

$$
\left(\mathbb{Q}\left[G^{\prime}\right] \otimes_{\mathbb{Q}} V\right)^{G} \cong \mathbb{Q}\left[G^{\prime}\right] \otimes_{\mathbb{Q}[G]} V
$$

as a left $\mathbb{Q}\left[G^{\prime}\right]$-module, where in the left hand side, $G$ acts on $\mathbb{Q}\left[G^{\prime}\right]$ via the inverse of the right regular representation.

The following propositions follow immediately from the proofs of their counterparts in [10], Proposition 4.1-4.

Proposition 7.4. For any object $X$ of $\left(G, \overline{\operatorname{Var}}_{S}\right)$, and any object $Y$ of $\left(G^{\prime}, \overline{\operatorname{Var}}_{S}\right)$, there is a natural bijection

$$
\operatorname{Hom}_{\left(G, \overline{V a r}_{S}\right)}\left(X, \operatorname{Res}_{\psi} Y\right) \cong \operatorname{Hom}_{\left(G^{\prime}, \overline{\operatorname{Var}}_{S}\right)}\left(\operatorname{Ind}_{\psi} X, Y\right)
$$

Proposition 7.5. The functors $\operatorname{Ind}_{\psi}:\left(G, C M o t_{S}\right) \rightarrow\left(G^{\prime}, C M o t_{S}\right)$ and $\operatorname{Ind}_{\psi}:$ $\mathrm{Ho}\left(G, \mathcal{C}^{b}\left(C M o t_{S}\right)\right) \rightarrow H o\left(G^{\prime}, \mathcal{C}^{b}\left(C M o t_{S}\right)\right)$ are left adjoint to Res $\psi$

Proposition 7.6. For any object $M$ in $\left(G, \mathcal{C}^{b}\left(C M o t_{S}\right)\right)$, and any object $N$ in $\left(G^{\prime}, \mathcal{C}^{b}\left(C M o t_{S}\right)\right)$, we have the projection formula

$$
\operatorname{Ind}_{\psi}\left(\operatorname{Res}_{\psi} N \otimes M\right) \cong N \otimes \operatorname{Ind}_{\psi} M
$$

Proposition 7.7. For any object $X$ of $\left(G, \overline{V a r}_{S}\right)$, and any object $Y$ of $\left(G^{\prime}, \overline{\operatorname{Var}}_{S}\right)$, we have the projection formula

$$
\operatorname{Ind}_{\psi}\left(\operatorname{Res}_{\psi} Y \otimes X\right) \cong Y \otimes \operatorname{Ind}_{\psi} X
$$

\subsection{The motive of a quotient variety.}

Theorem 7.8. The diagram

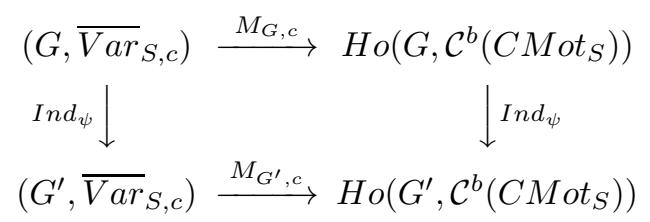

commutes.

In other words, for any object $X$ in $\left(G, \overline{V a r}_{S}\right)$, there exists a natural isomorphism $M_{G^{\prime}, c}\left(\operatorname{Ind}_{\psi}(X)\right) \cong \operatorname{Ind}_{\psi}\left(M_{G, c}(X)\right)$.

Proof. In the absolute case $S=\operatorname{Spec} k$, this is the main result of 10 . We will show that their arguments carry over the the relative case. The result will appear as a consequence of the uniqueness statement in Theorem 6.18 if we can prove that both paths in the diagram are isomorphic on $\left(G, \overline{\mathcal{V}}_{S}\right)$, and satisfy $(D)$ and $(E)$ on $\left(G, \overline{V a r}_{S, c}\right)$. This is done in Lemma 7.9 and Lemma 7.10 below.

Let $X$ be any $S$-variety with good $G$-action. The quotient map

$$
\pi: G^{\prime} \times X \rightarrow\left(G^{\prime} \times X\right) / G=\operatorname{Ind}_{\psi} X
$$

induces a morphism

$$
\pi^{*}: M_{c, G} \operatorname{Ind}_{\psi} X \rightarrow\left(\mathbf{1}\left[G^{\prime}\right] \otimes M_{G, c}(X)\right)^{G}
$$


and this defines a morphism of functors

$$
\psi: M_{c, G} \operatorname{Ind}_{\psi} \rightarrow \operatorname{Ind}_{\psi} M_{c, G}
$$

Lemma 7.9. The morphism $\psi$ is an isomorphism on $\left(G, \overline{\mathcal{V}}_{S}\right)$.

Proof. We will merely sketch the arguments in [10], to show that they carry over to our setting. We may suppose that $G^{\prime}=\{e\}$. Let $S$ be a smooth quasi-projective variety over $k$. The first step is to construct a category of effective Chow motives starting from $S$-varieties of the form $X^{\prime}=X / G$, with $X$ in $\left(G, \mathcal{V}_{S}\right)$. We denote the full subcategory of $\operatorname{Var}_{S}$ with these varieties as objects by $\mathcal{V}_{S}^{\prime}$. By [21, 17.4.10], the usual construction of Chow motives still makes sense if we start from $\mathcal{V}_{S}^{\prime}$ instead of $\mathcal{V}_{S}$, and we obtain a pseudo-abelian category $\mathcal{M}_{+}^{o}(S)^{\prime}$. There is an obvious fully faithful embedding

$$
\Phi: \mathcal{M}_{+}^{o}(S) \rightarrow \mathcal{M}_{+}^{o}(S)^{\prime}
$$

and we show that it is an equivalence by establishing an isomorphism between the motive of $X / G$ in $\mathcal{M}_{+}^{o}(S)^{\prime}$, and the image of $M(X)^{G}$ under $\Phi$. This is done as in [10, $1.2]$, using Manin's identity principle and the fact that $C H(X / G, \mathbb{Q}) \cong C H(X, \mathbb{Q})^{G}$ (see [21, 1.7.6]). We fix a quasi-inverse functor for $\Phi$, and this yields a functor

$$
M^{\prime}: \mathcal{V}_{S}^{\prime} \rightarrow \mathcal{M}_{+}^{o}(S): X / G \rightarrow M^{\prime}(X / G) \cong M(X)^{G}
$$

If $S$ is any $k$-variety, taking direct limits over admissible stratifications of $S$ yields a functor

$$
M^{\prime}: \overline{\mathcal{V}}_{S}^{\prime} \rightarrow \mathrm{CMot}_{S}
$$

If we define elementary acyclic diagrams in $\overline{\mathcal{V}}_{S}^{\prime}$ as quotients of elementary acyclic diagrams in $\left(G, \overline{\mathcal{V}}_{S}\right)$, then we can formulate an extension principle for the category $\overline{\mathcal{V}}_{S}^{\prime}$ as in Theorem 6.18 (see [10, 2.2] for the absolute case). In particular, $M^{\prime}$ has an essentially unique extension

$$
M_{c}^{\prime}: \overline{\operatorname{Var}}_{S, c} \rightarrow H o\left(\mathcal{C}^{b}\left(C M o t_{S}\right)\right)
$$

satisfying $(D)$ and $(E)$. However, is is easily seen that $\psi$ defines an isomorphism of functors $M \cong M^{\prime}$ on $\overline{\mathcal{V}}_{S}$, so by the uniqueness statement in Theorem 6.18 $\psi$ is also an isomorphism on $\overline{\mathcal{V}}_{S}^{\prime}$. This concludes the proof.

Lemma 7.10. The functors

$$
M_{c, G} \operatorname{Ind}_{\psi} \text { and } \operatorname{Ind}_{\psi} M_{c, G}:\left(G, \overline{\operatorname{Var}}_{S, c}\right) \rightarrow H o\left(G^{\prime}, \mathcal{C}^{b}\left(C M o t_{S}\right)\right)
$$

satisfy properties $(D)$ and $(E)$ from Theorem 6.18 . Hence,

$$
\psi: M_{c, G} \operatorname{Ind}_{\psi} \rightarrow \operatorname{Ind}_{\psi} M_{c, G}
$$

is an isomorphism of functors on $\overline{\operatorname{Var}}_{S, c}$.

Proof. We know that $M_{c, G}$ and $M_{c, G^{\prime}}$ respect acyclic diagrams, by construction. It is easy to see that $I n d_{\psi}$ respects acyclic diagrams of varieties, and acyclic diagrams in the descent categories (it commutes with $s$ since it is an additive functor).

The fact that $\psi$ is an isomorphism follows from Lemma 7.9 and the uniqueness statement in Theorem 6.18.

Corollary 7.11. For any $X$ in $\left(G, \overline{\operatorname{Var}}_{S}\right), M_{c}(X / G) \cong M_{G, c}(X)^{G}$. 
7.3. Character decomposition. We denote by $C(G, \mathbb{Q})$ the $\mathbb{Q}$-vector space of $\mathbb{Q}$-central functions, i.e. the space of $\mathbb{Q}$-linear combinations of characters of $\mathbb{Q}$ irreducible representations of $G$. Recall that a central function $\alpha: G \rightarrow \mathbb{Q}$ belongs to $C(G, \mathbb{Q})$ iff $\alpha(x)=\alpha\left(x^{\prime}\right)$ for each pair $\left\{x, x^{\prime}\right\}$ of elements of $G$, for which the subgroups generated by $x$, resp. $x^{\prime}$, are conjugate in $G$. Artin proved that every such $\alpha$ is a $\mathbb{Q}$-linear combination of characters of the form $\operatorname{In} d_{H}^{G} 1_{H}$, with $H$ a cyclic subgroup of $G$, and $1_{H}$ the trivial character on $H$.

Definition 7.12. For any finite dimensional $\mathbb{Q}$-vector space $V$ with $G$-action, we define an element $V$ of $\left(G, C M o t_{S}\right)$ as follows: we write the vector space $V$ as the image of $\mathbb{Q}[G]^{n}$ under a projector $p$, and we define the object $V$ of $C M o t_{S}$ as the image of the corresponding projector on $\mathbf{1}[G]^{\oplus n}$.

Lemma 7.13. For any pair of objects $M, N$ of $\left(G, C M\right.$ ot $\left._{S}\right)$, and any finite $\mathbb{Q}[G]$ module $V$, we have a natural isomorphism of $\mathbb{Q}[G]$-modules

$$
\operatorname{Hom}_{C M o t_{S}}(N, V \otimes M) \cong V \otimes_{\mathbb{Q}} \operatorname{Hom}_{C M o t_{S}}(N, M)
$$

Proof. If $V$ is the image of $\mathbb{Q}[G]^{n}$ under a projector $p$, we have

$$
\begin{aligned}
\operatorname{Hom}_{C M o t}(N, V \otimes M) & =(p \otimes I d) \circ H_{\operatorname{lom}_{C M o t}}\left(N, \mathbf{1}[G]^{\oplus n} \otimes M\right) \\
& =p\left(\mathbb{Q}[G]^{n}\right) \otimes_{\mathbb{Q}} \operatorname{Hom}_{C M o t}(N, M) \\
& =V \otimes_{\mathbb{Q}} \operatorname{Hom}_{C M o t}(N, M)
\end{aligned}
$$

Definition 7.14. Let $\alpha$ be an effective character of $G$ over $\mathbb{Q}$, and let $\rho_{\alpha}: G \rightarrow$ $G L\left(V_{\alpha}\right)$ be the corresponding representation. We denote by $V_{\alpha}^{\vee}$ its dual representation, as well as the associated object in $\left(G, C M o t_{S}\right)$. For any object $M$ of $\left(G, C M o t_{S}\right)$, we put

$$
M_{\alpha}:=\left(V_{\alpha}^{\vee} \otimes M\right)^{G}
$$

in $\mathrm{CMot}_{S}$. This defines additive functors, all denoted by $(.)_{\alpha}$

$$
\begin{aligned}
\left(G, C_{\text {Mot }}\right) & \rightarrow C M \text { Mot }_{S} \\
\left(G, \mathcal{C}^{b}\left(\text { CMot }_{S}\right)\right) & \rightarrow \mathcal{C}^{b}\left(\text { CMot }_{S}\right) \\
H o\left(G, \mathcal{C}^{b}\left(\text { CMot }_{S}\right)\right) & \rightarrow H o\left(\mathcal{C}^{b}\left(\text { CMot }_{S}\right)\right)
\end{aligned}
$$

Lemma 7.15. For any $\mathbb{Q}$-irreducible character $\alpha$, and any pair of objects $M, N$ of $\left(G, C M\right.$ ot $\left._{S}\right)$, we have a natural isomorphism of $\mathbb{Q}[G]$-modules

$$
\operatorname{Hom}_{C M o t}\left(N, M_{\alpha}\right) \cong \operatorname{Hom}_{\mathbb{Q}[G]}\left(V_{\alpha}, \operatorname{Hom}_{C M o t}(N, M)\right)
$$

Proof.

$$
\begin{aligned}
\operatorname{Hom}_{C M o t}\left(N, M_{\alpha}\right) & \cong\left(V_{\alpha}^{\vee} \otimes_{\mathbb{Q}} \operatorname{Hom}_{C \operatorname{Mot}_{S}}(N, M)\right)^{G} \\
& \cong \operatorname{Hom}_{\mathbb{Q}}\left(V_{\alpha}, \operatorname{Hom}_{C \operatorname{Mot}_{S}}(N, M)\right)^{G} \\
& \cong \operatorname{Hom}_{\mathbb{Q}[G]}\left(V_{\alpha}, \operatorname{Hom}_{C M \cot _{S}}(N, M)\right)
\end{aligned}
$$

Suppose that $\alpha$ is $\mathbb{Q}$-irreducible, of degree $n_{\alpha}$. We define an idempotent $p_{\alpha}$ in the group algebra $\mathbb{Q}[G]$ by

$$
p_{\alpha}:=\frac{n_{\alpha}}{|G|<\alpha, \alpha>} \sum_{g \in G} \alpha\left(g^{-1}\right)[g]
$$


Proposition 7.16. For each $\alpha \in C(G, \mathbb{Q})$, there exists a unique morphism of abelian groups

$$
\chi_{c, \alpha}: K_{0}^{G}\left(\operatorname{Var}_{S}\right) \rightarrow K_{0}\left(\operatorname{CMot}_{S}\right) \otimes \mathbb{Q}
$$

such that

(i) if $X$ belongs to $\left(G, \mathcal{V}_{T}\right)$, for some smooth, irreducible, and quasi-projective locally closed subset $T$ of $S$, and if $\alpha$ is a $\mathbb{Q}$-irreducible character on $G$, then

$$
\frac{n_{\alpha}}{<\alpha, \alpha>} \chi_{c, \alpha}([X])
$$

is equal to the class of the image of the projector $p_{\alpha}$ on the object $M(X)$ of $\mathcal{M}_{+}^{o}(T)$. (ii) the morphism $\chi_{c, \alpha}$ is $\mathbb{Q}$-linear in $\alpha$.

Proof. We can construct $\chi_{c, \alpha}$ as follows: if $\alpha$ is effective, we map the class of an $S$-variety $X$ with good $G$-action in $K_{0}^{G}\left(\operatorname{Var}_{S}\right)$, to the class of $\left(M_{G, c}(X)\right)_{\alpha}$ in $K_{0}\left(C M t_{S}\right)$. Property (ii) is clear, so let us prove $(i)$. For any object $N$ in $C M o t_{S}$, we have

$$
\operatorname{Hom}_{C M o t_{S}}\left(N,\left.\operatorname{Im} p_{\alpha}\right|_{M(X)}\right) \cong p_{\alpha} \mathbb{Q}[G] \otimes_{\mathbb{Q}[G]} \operatorname{Hom}(N, M(X))
$$

In view of Lemma 7.15 and Yoneda's Lemma, any choice of an isomorphism of $\mathbb{Q}$-vector spaces

$$
\mathbb{Q}[G] \cong\left(V_{\alpha}^{\vee}\right)^{\oplus n_{\alpha} /<\alpha, \alpha>}
$$

induces an isomorphism

$$
\operatorname{Im}_{p_{\alpha}} \cong\left(M(X)_{\alpha}\right)^{\oplus n_{\alpha} /<\alpha, \alpha>}
$$

in $\mathrm{CMot}_{S}$.

Alternatively, we can take point $(i)$ as a definition, and use Bittner's presentation of $K_{0}^{G}\left(\operatorname{Var}_{S}\right)$ (see Remark 6.10 in Section 6.10) and Theorem 6.12

Lemma 7.17. Let $G_{1}$ and $G_{2}$ be finite groups, and let $\alpha_{i}$ be an element of $C\left(G_{i}, \mathbb{Q}\right)$, for $i=1,2$. Consider the ring morphism

$$
\Delta: K_{0}^{G_{1}}\left(\operatorname{Var}_{S}\right) \otimes_{\mathbb{Z}} K_{0}^{G_{2}}\left(\operatorname{Var}_{S}\right) \rightarrow K_{0}^{G_{1} \times G_{2}}\left(\operatorname{Var}_{S}\right)
$$

obtained as follows: the projection of $G_{1} \times G_{2}$ on $G_{i}$ induces a morphism $\pi_{i}$ : $K_{0}^{G_{i}}\left(\operatorname{Var}_{S}\right) \rightarrow K_{0}^{G_{1} \times G_{2}}\left(\operatorname{Var}_{S}\right)$. The composition of

$$
\pi_{1} \times \pi_{2}: K_{0}^{G_{1}}\left(\operatorname{Var}_{S}\right) \times K_{0}^{G_{2}}\left(\operatorname{Var}_{S}\right) \rightarrow K_{0}^{G_{1} \times G_{2}}\left(\operatorname{Var}_{S}\right) \times K_{0}^{G_{1} \times G_{2}}\left(\operatorname{Var}_{S}\right)
$$

with ring multiplication in $K_{0}^{G_{1} \times G_{2}}\left(\operatorname{Var}_{S}\right)$, is bilinear, and induces the morphism $\Delta$. We have

$$
\chi_{c, \alpha_{1}} \otimes \chi_{c, \alpha_{2}}=\chi_{c, \alpha_{1} \cdot \alpha_{2}} \circ \Delta
$$

Proof. Let $T$ be any smooth, quasi-projective, irreducible locally closed subset of $S$. Let $X_{i}$ be a proper and smooth variety over $T$, with good $G_{i}$-action, for $i=1,2$. We may assume $\alpha_{i}$ is $\mathbb{Q}$-irreducible, for $i=1,2$. Let $\rho_{i}: G_{i} \rightarrow G L\left(V_{i}\right)$ be the representation with character $\alpha_{i}$. The external tensor product $\rho_{1} \otimes \rho_{2}: G_{1} \times G_{2} \rightarrow$ $V_{1} \otimes_{\mathbb{Q}} V_{2}$ is an irreducible representation with character $\alpha_{1} \cdot \alpha_{2}$.

Hence, by Proposition $7.16(i)$, it suffices to show that the projectors $\operatorname{Im} p_{\alpha_{1} . \alpha_{2}}$ and $\operatorname{Im} p_{\alpha_{1}} \otimes I m p_{\alpha_{2}}$ on $M\left(X_{1} \times_{T} X_{2}\right)$ have isomorphic images in $\mathcal{M}_{+}^{o}(T)$. This, however, is clear.

Now we prove two Frobenius reciprocity properties. 
Lemma 7.18. Let $\psi: G \rightarrow G^{\prime}$ be a morphism of finite groups. For any $S$-variety $X$ with good $G$-action, and any $\alpha \in C\left(G^{\prime}, \mathbb{Q}\right)$,

$$
\chi_{c, \alpha}\left(\left[\operatorname{Ind}_{\psi} X\right]\right)=\chi_{c, \operatorname{Res}_{\psi} \alpha}([X])
$$

in $K_{0}\left(\mathrm{CMot}_{S}\right) \otimes \mathbb{Q}$.

Proof. We may assume that $\alpha$ is effective. By definition,

$$
M_{G^{\prime}, c}\left(\operatorname{Ind}_{\psi} X\right)_{\alpha}=\left(V_{\alpha}^{\vee} \otimes M_{G^{\prime}, c}\left(\operatorname{Ind}_{\psi} X\right)\right)^{G^{\prime}}
$$

By Proposition 7.8 this object is isomorphic to

$$
\left(V_{\alpha}^{\vee} \otimes I n d_{\psi} M_{G, c}(X)\right)^{G^{\prime}}
$$

By the projection formula in Proposition 7.7 we get

$$
\begin{aligned}
\left(V_{\alpha}^{\vee} \otimes \operatorname{Ind}_{\psi} M_{G, c}(X)\right)^{G^{\prime}} & \cong\left(\operatorname{Ind}_{\psi}\left(\operatorname{Res}_{\psi} V_{\alpha}^{\vee} \otimes M_{G, c}(X)\right)\right)^{G^{\prime}} \\
& \cong\left(V_{\operatorname{Res}_{\psi} \alpha} \otimes M_{G, c}(X)\right)^{G} \\
& \cong M_{G, c}(X)_{\operatorname{Res}_{\psi} \alpha}
\end{aligned}
$$

Corollary 7.19 (Relative motive of a quotient variety). Let $X$ be an $S$-variety with good $G$-action, and let $H$ be a normal subgroup of $G$. For every $\alpha$ in $C(G / H, \mathbb{Q})$,

$$
\chi_{c, \alpha}([X / H])=\chi_{c, \alpha \circ \rho}([X])
$$

where $\rho$ is the projection $\rho: G \rightarrow G / H$.

Proof. Apply Lemma 7.18 to the projection $\psi: G \rightarrow G / H$.

Lemma 7.20. Let $\psi: G \rightarrow G^{\prime}$ be a morphism of finite groups. For any $S$-variety $X$ with good $G^{\prime}$-action, and any $\alpha \in C(G, \mathbb{Q})$,

$$
\chi_{c, \alpha}\left(\left[\operatorname{Res}_{\psi} X\right]\right)=\chi_{c, I n d_{\psi} \alpha}([X])
$$

in $K_{0}\left(C M o t_{S}\right) \otimes \mathbb{Q}$.

Proof. We may assume that $\alpha$ is effective. By definition,

$$
\begin{aligned}
M_{G^{\prime}, c}(X)_{I n d_{\psi} \alpha} & \cong\left(V_{I n d_{\psi} \alpha}^{\vee} \otimes M_{G^{\prime}, c}(X)\right)^{G^{\prime}} \\
& \cong\left(I n d_{\psi} V_{\alpha}^{\vee} \otimes M_{G^{\prime}, c}(X)\right)^{G^{\prime}}
\end{aligned}
$$

By the projection formula in Proposition 7.7 we get

$$
\begin{aligned}
\left(\operatorname{Ind}_{\psi} V_{\alpha}^{\vee} \otimes M_{G^{\prime}, c}(X)\right)^{G^{\prime}} & \cong\left(\operatorname{Ind}_{\psi}\left(V_{\alpha}^{\vee} \otimes \operatorname{Res}_{\psi} M_{G^{\prime}, c}(X)\right)\right)^{G^{\prime}} \\
& \cong\left(V_{\alpha}^{\vee} \otimes \operatorname{Res}_{\psi} M_{G^{\prime}, c}(X)\right)^{G} \\
& \cong M_{G, c}\left(\operatorname{Res}_{\psi} X\right)_{\alpha}
\end{aligned}
$$

where the last isomorphism follows from Proposition 7.1

Corollary 7.21. For every $S$-variety $X$ with good $G$-action,

$$
\chi_{c}([X])=\sum_{\alpha} \frac{n_{\alpha}}{<\alpha, \alpha>} \chi_{c, \alpha}(X)
$$

where we take the sum over all $\mathbb{Q}$-irreducible representations $\alpha$.

Proof. The regular character $\chi_{r e g}$ on $G$ is induced by the trivial character on the trivial subgroup. Now use Lemma 7.20, and the fact that for each $\mathbb{Q}$-irreducible character $\alpha$, its degree $n_{\alpha}$ equals its multiplicity in $\chi_{r e g}$ times $\langle\alpha, \alpha\rangle$. 
Definition 7.22. We denote by $K_{0}^{m o t}\left(\operatorname{Var}_{S}\right)$ the image of the morphism

$$
\chi_{c}: K_{0}\left(\operatorname{Var}_{S}\right) \rightarrow K_{0}\left(\mathrm{CMot}_{S}\right)
$$

constructed in Corollary 6.21,

Lemma 7.23. For any $\mathbb{Q}$-central function $\alpha$ in $C(G, \mathbb{Q})$, the image of $\chi_{c, \alpha}$ is contained in $K_{0}^{\text {mot }}\left(\operatorname{Var}_{S}\right) \otimes \mathbb{Q}$.

Proof. In view of Artin's result mentioned above, it suffices to prove this lemma when $\alpha$ is of the form $\operatorname{In} d_{H}^{G} 1_{H}$, where $H$ is a cyclic subgroup of $G$, and $1_{H}$ is the trivial character on $H$. By Lemma 7.20 we may assume that $G$ is cyclic, and $G=H$. In this case, Lemma 7.23 is an immediate consequence of Corollary 7.19

Lemma 7.24 (Base Change). Let $f: T \rightarrow S$ be a morphism of $k$-varieties. For any $\alpha \in C(G, \mathbb{Q})$, the base change square

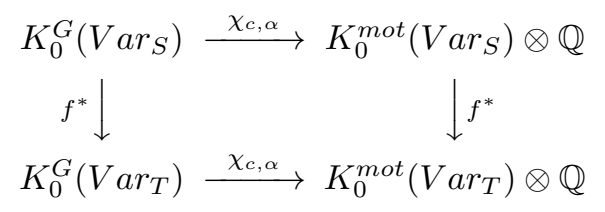

commutes.

Proof. This is clear from the definition.

\section{A motivic incARnAtion For the RELATIVE theory of PSEUdo-Finite} FIELDS

Now we're ready to construct the relative counterpart of the morphism

$$
\chi_{(c)}: K_{0}\left(P F F_{k}\right) \rightarrow K_{0}^{m o t}\left(\operatorname{Var}_{k}\right) \otimes \mathbb{Q}
$$

from the introduction. Throughout this section, our base scheme $S$ is a variety over a field $k$ of characteristic zero.

Definition 8.1. Let $X$ be an affine normal irreducible variety over $S$, let $Y$ be a Galois cover of $X$, and let $C o n$ be a conjugation domain of the cover $Y / X$. Put $G=G(Y / X)$. We define a $\mathbb{Q}$-central function $\alpha_{C o n}$ in $C(G, \mathbb{Q})$ as follows: $\alpha(g)=1$ if the subgroup generated by $g$ belongs to Con, and $\alpha(g)=0$ else.

Let $\Theta$ be the set of quantifier-free Galois formulas $\theta$ over $S$. We define a map

$$
\chi_{(c)}: \Theta \rightarrow K_{0}^{m o t}\left(\operatorname{Var}_{S}\right) \otimes \mathbb{Q}
$$

as follows: for any quantifier-free Galois formula $\theta$ over $S$, corresponding to a Galois stratification $<X, C_{i} / A_{i}, \operatorname{Con}\left(A_{i}\right)>$, we define $\chi_{(c)}(\theta)$ by

$$
\chi_{(c)}(\theta):=\sum_{i} \chi_{c, \alpha_{C o n}\left(A_{i}\right)}\left(\left[C_{i}\right]\right)
$$

Lemma 8.2. Let $\theta$ and $\theta^{\prime}$ be quantifier-free Galois formulas, such that the Galois stratification corresponding to $\theta^{\prime}$ is obtained from the one corresponding to $\theta$ by inflation. Then

$$
\chi_{(c)}(\theta)=\chi_{(c)}\left(\theta^{\prime}\right)
$$


Proof. Let $X, Y$ be affine normal irreducible varieties over $S$, let $Y / X$ be a Galois cover, and let $\operatorname{Con}(Y / X)$ be a conjugation domain for this cover. Put $G^{\prime}=$ $G(Y / X)$. Let $Z / X$ be a cover dominating $Y / X$, put $G=G(Z / X)$, and denote by $\operatorname{Con}(Z / X)$ the conjugation domain obtained by inflation. Denote by $\psi: G \rightarrow G^{\prime}$ the projection.

We have $Y=I n d_{\psi} Z$, and hence, by Lemma 7.18 it suffices to observe that $\alpha_{C o n(Z / X)}=\operatorname{Res}_{\psi} \alpha_{C o n}(Y / X)$.

Lemma 8.3. Let $\theta$ and $\theta^{\prime}$ be quantifier-free Galois formulas, such that the Galois stratification corresponding to $\theta^{\prime}$ is obtained from the one corresponding to $\theta$ by refinement. Then

$$
\chi_{(c)}(\theta)=\chi_{(c)}\left(\theta^{\prime}\right)
$$

Proof. Let $X, Y$ be affine normal irreducible varieties over $S$, let $Y / X$ be a Galois cover, and let $\operatorname{Con}(Y / X)$ be a conjugation domain for this cover. Put $G^{\prime}=$ $G(Y / X)$. Let $U$ be a normal irreducible closed subvariety of $X$, and let $V$ be any connected component of $Y_{U}=Y \times_{X} U$. Put $G=G(V / U)$, and let $\psi: G \rightarrow G^{\prime}$ be the inclusion. Let $\operatorname{Con}(V / U)$ be the conjugation domain induced from $\operatorname{Con}(Y / X)$ by refinement.

Since $Y_{U}=\operatorname{Ind}_{\psi} V$ and $\alpha_{C o n(V / U)}=\operatorname{Res}_{\psi} \alpha_{C o n(Y / X)}$, we see from Lemma 7.18 that

$$
M_{c, G}\left(Y_{U}\right)_{\alpha_{C o n(Y / X)}} \cong M_{c, G^{\prime}}(V)_{\alpha_{C o n(V / U)}}
$$

The result now follows from additivity of $M_{c, G}$.

Lemma 8.4. Let $m$ be a positive integer, let $\mathcal{A}$ and $\mathcal{B}$ be Galois stratifications of $\mathbb{A}_{S}^{m}$, and suppose that there exists a Galois stratification $\mathcal{G}$ of $\mathbb{A}_{S}^{m} \times_{S} \mathbb{A}_{S}^{m}$, such that, for each point $x$ of $S$, and each pseudo-finite field extension $M$ of $k(x)$, the set $Z(\mathcal{G}, x, M)$ is the graph of a bijection between $Z(\mathcal{A}, x, M)$ and $Z(\mathcal{B}, x, M)$. Let $\theta_{A}$, $\theta_{B}$, and $\theta_{G}$ be the quantifier-free Galois formulas corresponding to $\mathcal{A}, \mathcal{B}$, resp. $\mathcal{G}$. Then

$$
\chi_{(c)}\left(\theta_{A}\right)=\chi_{(c)}\left(\theta_{B}\right)=\chi_{(c)}\left(\theta_{G}\right)
$$

Proof. It suffices to prove that $\chi_{(c)}\left(\theta_{A}\right)=\chi_{(c)}\left(\theta_{G}\right)$. Refining our stratifications, by Lemma 8.3 . we may suppose that $\mathcal{A}$ contains at most one stratum $C / A$ with non-empty conjugation domain $\operatorname{Con}(A)$, and we may restrict $\mathcal{G}$ to a stratification of $A \times{ }_{S} \mathbb{A}_{S}^{m}$. Write $\mathcal{G}$ as $<\mathbb{A}_{S}^{m} \times{ }_{S} \mathbb{A}_{S}^{m}, D_{i} / G_{i}, \operatorname{Con}\left(G_{i}\right)>$. Let $W$ be the support of $\mathcal{G}$, i.e. the union of the strata $G_{i}$ with non-empty conjugation domain, and let $\pi: W \rightarrow A$ be the projection.

First, suppose that the support of $\mathcal{A}$ is empty, i.e. that $\operatorname{Con}(A)$ is empty. Since $\mathcal{G}$ defines the graph of a bijection between $\mathcal{A}$ and $\mathcal{B}$, this means that $Z(\mathcal{G}, x, M)$ is empty, for any point $x$ of $S$, and any pseudo-finite field $M$ containing $k(x)$. By Lemma 2.8 this implies that the support of $\mathcal{G}$ is empty. Hence, $\chi_{(c)}\left(\theta_{A}\right)=$ $\chi_{(c)}\left(\theta_{G}\right)=0$.

So we may assume that that $\operatorname{Con}(A)$ is not empty. By Lemma 2.8 the union of the sets $Z(\mathcal{A}, x, M)$, where $x$ runs over the points of $A$, and $M$ runs over the pseudo-finite field extensions of $k(x)$, is dense in $A$. Since $\pi$ induces, for each $x$ and each $M$, a bijection between $Z(\mathcal{A}, x, M)$ and $Z(\mathcal{G}, x, M)$, this shows that the image of $\pi$ is dense in $A$.

Now suppose that $G$ is a stratum of $W$, and $a$ is a closed point of $A$, such that the fiber of $G$ over $a$ has dimension $>0$. After a refinement, we may suppose that $G$ is 
mapped to $a$. Let $x$ be the image of $a$ in $S$. By Lemma 2.8 the conjugation domain of $G$ must be empty; if not, $Z(\mathcal{G}, x, M)$ would contain infinitely many points lying over $a$, for some pseudo-finite field $M$ containing $k(x)$.

Hence, there exists an open dense subscheme $A^{\prime}$ of $A$, such that the restriction of $\mathcal{G}$ to $\pi^{-1}\left(A^{\prime}\right)$ satisfies the following property: for every stratum $G_{i}$ of this restriction, $\pi: G_{i} \rightarrow A^{\prime}$ is étale and finite. By Noetherian induction, we might as well assume that $A=A^{\prime}$.

We can dominate the étale cover $C / A$ and all the composed covers $D_{i} / A$ by a common Galois cover $D / A$. By Lemma 8.2 we might as well assume that $D=$ $C=D_{i}$ for all $i$. We will show that

$$
\alpha_{C o n(A)}=\sum_{i} \operatorname{Ind} d_{G\left(D / G_{i}\right)}^{G(D / A)} \alpha_{C o n\left(G_{i}\right)}
$$

This will complete the proof, by Lemma 7.20

Let $M$ be any field, and let $a$ be an $M$-valued point on $A$. The point $a$ lifts to an $M$-valued point $b$ on $G_{i}$, for some $i$, iff $C_{D / A}(a) \cap G\left(D / G_{i}\right)$ is not empty, by Lemma 2.4. In this case, there exists a group $H$ in this intersection, such that $C_{D / G_{i}}(b)$ is the conjugation class of $H$ in $G\left(D / G_{i}\right)$.

Conversely, if $H^{\prime}$ is a subgroup of $G\left(D / G_{i}\right)$ for some $i$, and if $b$ is an $M$-valued point on $G_{i}$ with $H^{\prime} \in C_{D / G_{i}}(b)$, then the image of $b$ in $A$ is an $M$-valued point $a$ whose decomposition class $C_{D / A}(a)$ is the conjugation class of $H^{\prime}$ in $G(D / A)$.

Let $g$ be any element of $G(D / A)$, denote by $H$ the subgroup of $G(D / A)$ generated by $g$, and denote by $C_{H}$ its conjugation class in $G(D / A)$. By definition, $\alpha_{C o n(A)}(g)=1$ if there exists a point $x$ on $S$, a pseudo-finite field $M$ containing $k(x)$, and a point $a$ in $Z(\mathcal{A}, x, M)$ with $H \in C_{D / A}(a)$. Else, $\alpha_{C o n(A)}(g)=0$.

In the latter case, $H \notin C o n(A)$, and since $\mathcal{G}$ defines a bijection between $\mathcal{A}$ and $\mathcal{B}, C_{H} \cap \operatorname{Con}\left(G_{i}\right)$ is empty, for all $i$. This means that both members of (3) vanish when evaluated in $g$.

In the first case, there exists a unique index $i$ such that $a$ lifts to an $M$-valued point $b$ on $G_{i}$, with $C_{D / G_{i}}(b) \subset \operatorname{Con}\left(G_{i}\right)$, and this point $b$ is also unique. This means that there exists a unique index $i$ such that $C_{D / A}(a) \cap \mathscr{P}\left(G\left(D / G_{i}\right)\right)$ is nonempty and contained in $\operatorname{Con}\left(G_{i}\right)$, and moreover, $C_{D / A}(a) \cap \operatorname{Con}\left(G_{i}\right)$ consists of a single conjugation class in $G\left(D / G_{i}\right)$. Hence, both members of (3i) are equal to 1 when evaluated in $g$.

By Lemma 8.4 and the Elimination Theorem 4.1 the map $\chi_{(c)}$ from Definition 8.1 factors through a map of sets

$$
\chi_{(c)}: K_{0}\left(P F F_{S}\right) \rightarrow K_{0}^{m o t}\left(\operatorname{Var}_{S}\right) \otimes \mathbb{Q}
$$

We now show that it respects the ring structures. By Lemma 8.4 we may freely identify Galois formulas $\theta$ with equivalent Galois formulas or equivalent ring formulas. In particular, $\chi_{(c)}$ is well-defined on $\theta_{1} \times \theta_{2}, \theta_{1} \vee \theta_{2}$ and $\theta_{1} \wedge \theta_{2}$, for any pair of Galois formulas $\theta_{1}, \theta_{2}$.

Lemma 8.5. Let $\theta_{1}$ and $\theta_{2}$ be quantifier-free Galois formulas corresponding to Galois stratifications $\mathcal{A}_{1}$ and $\mathcal{A}_{2}$, and denote by $\theta_{1} \times{ }_{S} \theta_{2}$ the Galois formula corresponding to the product $\mathcal{A}_{1} \times_{S} \mathcal{A}_{2}$. Then

$$
\chi_{(c)}\left(\theta_{1} \times \theta_{2}\right)=\chi_{(c)}\left(\theta_{1}\right) \cdot \chi_{(c)}\left(\theta_{2}\right)
$$


Proof. Let $X_{1}, X_{2}$ be affine normal irreducible varieties over $S$, let $Y_{1} / X_{1}$ and $Y_{2} / X_{2}$ be Galois covers, and let $C_{o n}$, resp. $C n_{2}$ be conjugation domains for these covers. Put $G_{i}=G\left(Y_{i} / X_{i}\right)$ for $i=1,2$.

Let $Z$ be any connected component of $Y_{1} \times_{S} Y_{2}$, and let $C o n$ be the conjugation domain for the cover $Z / X_{1} \times_{S} X_{2}$ defined in Section 3.8 Put

$$
G=G\left(Z / X_{1} \times{ }_{S} X_{2}\right)
$$

and denote by $\psi: G_{1} \times G_{2} \rightarrow G$ the projection. Observe that

$$
\operatorname{Res}_{\psi} \alpha_{C o n}=\alpha_{C o n_{1}} \cdot \alpha_{C o n_{2}}
$$

in $C\left(G_{1} \times G_{2}, \mathbb{Q}\right)$. By Lemma 7.17 and Lemma 7.18

$$
M_{c, G}(Z)_{\alpha_{C o n}} \cong M_{c, G_{1}}\left(Y_{1}\right)_{\alpha_{C o n_{1}}} \otimes M_{c, G_{2}}\left(Y_{2}\right)_{\alpha_{C o n_{2}}}
$$

Lemma 8.6. For any pair of quantifier-free Galois formulas $\theta_{1}, \theta_{2}$ with the same free variables, we have

$$
\chi_{(c)}\left(\theta_{1} \vee \theta_{2}\right)+\chi_{(c)}\left(\theta_{1} \wedge \theta_{2}\right)=\chi_{(c)}\left(\theta_{1}\right)+\chi_{(c)}\left(\theta_{2}\right)
$$

For any pair of quantifier-free Galois formulas $\theta_{1}, \theta_{2}$ with disjoint sets of free variables, we have

$$
\chi_{(c)}\left(\theta_{1} \wedge \theta_{2}\right)=\chi_{(c)}\left(\theta_{1}\right) \cdot \chi_{(c)}\left(\theta_{2}\right)
$$

Proof. Let $\theta_{1}$ and $\theta_{2}$ be Galois formulas corresponding to Galois stratifications $\mathcal{A}_{1}$ and $\mathcal{A}_{2}$.

First, suppose $\theta_{1}$ and $\theta_{2}$ have the same free variables. After a refinement, and using Lemma 8.3 we may suppose that the underlying stratifications of $\mathcal{A}_{1}$ and $\mathcal{A}_{2}$ coincide. If $Z\left(\theta_{1} \wedge \theta_{2}, x, M\right)$ is empty, for any point $x$ of $S$ and any pseudo-finite field extension $M$ of $k(x)$, the conjugation domains of $\mathcal{A}_{1}$ and $\mathcal{A}_{2}$ are disjoint, for each stratum, by Corollary 4.5 In this case, it is easy to see that

$$
\chi_{(c)}\left(\theta_{1} \vee \theta_{2}\right)=\chi_{(c)}\left(\theta_{1}\right)+\chi_{(c)}\left(\theta_{2}\right)
$$

In general, we have

$$
\begin{aligned}
\chi_{(c)}\left(\theta_{1} \vee \theta_{2}\right) & =\chi_{(c)}\left(\theta_{1} \wedge \theta_{2}\right)+\chi_{(c)}\left(\neg \theta_{1} \wedge \theta_{2}\right)+\chi_{(c)}\left(\theta_{1} \wedge \neg \theta_{2}\right) \\
\chi_{(c)}\left(\theta_{1}\right) & =\chi_{(c)}\left(\theta_{1} \wedge \theta_{2}\right)+\chi_{(c)}\left(\theta_{1} \wedge \neg \theta_{2}\right) \\
\chi_{(c)}\left(\theta_{2}\right) & =\chi_{(c)}\left(\theta_{1} \wedge \theta_{2}\right)+\chi_{(c)}\left(\neg \theta_{1} \wedge \theta_{2}\right)
\end{aligned}
$$

Hence,

$$
\chi_{(c)}\left(\theta_{1} \vee \theta_{2}\right)+\chi_{(c)}\left(\theta_{1} \wedge \theta_{2}\right)=\chi_{(c)}\left(\theta_{1}\right)+\chi_{(c)}\left(\theta_{2}\right)
$$

if $\theta_{1}$ and $\theta_{2}$ have the same free variables.

If $\theta_{1}$ and $\theta_{2}$ have disjoint sets of free variables, the formula $\theta_{1} \wedge \theta_{2}$ corresponds to the product $\mathcal{A}_{1} \times{ }_{S} \mathcal{A}_{2}$. By Lemma 8.5 we have

$$
\chi_{(c)}\left(\theta_{1} \wedge \theta_{2}\right)=\chi_{(c)}\left(\theta_{1}\right) \cdot \chi_{(c)}\left(\theta_{2}\right)
$$

In analogy with [15], Theorem 2.1, we state 
Theorem 8.7. There exists a unique ring morphism

$$
\chi_{(c)}: K_{0}\left(P F F_{S}\right) \rightarrow K_{0}^{m o t}\left(\operatorname{Var}_{S}\right) \otimes \mathbb{Q}
$$

satisfying the following two properties:

(i) for every quantifier-free ring formula $\varphi$, the image of $[\varphi]$ under $\chi_{(c)}$ equals $\chi_{c}([X])$, where $X$ is the $S$-constructible set defined by $\varphi$.

(ii) let $X$ be a normal affine irreducible variety over $S$, let $Y$ be a Galois cover of $X$, and let $C$ be a cyclic subgroup of the Galois group $G$ of the cover $Y / X$. To these data, we can associate a ring formula $\varphi_{Y / X, C}$ over $S$ (see Section (3.3), whose interpretation, in any point $x$ of $S$, and for any field $K$ containing $k(x)$, is the set of $K$-valued points $a \in X_{x}(K)$ with $C \in C_{Y / X}(a)$. Then

$$
\chi_{c}\left(\left[\varphi_{Y / X, C}\right]\right)=\frac{|C|}{\left|N_{G}(C)\right|} \chi_{(c)}\left(\left[\varphi_{Y /(Y / C), C}\right]\right),
$$

where $N_{G}(C)$ denotes the normalizer of $C$ in $G$.

Proof. 1. Uniqueness: By Corollary [4.6 the morphism $\chi_{(c)}$ is determined by the images of classes of the form $\left[\varphi_{Y / X, C}\right]$, with $C$ cyclic. Hence, by $(i i), \chi_{c}$ is determined by the images of classes of the form $\left[\varphi_{Y /(Y / C), C}\right]$, where $C$ is cyclic. However, by (i) and (ii),

$$
|C| \chi_{c}([Y / C])=\sum_{\text {A subgroup of } C}|A| \chi_{(c)}\left(\left[\varphi_{Y /(Y / A), A}\right]\right)
$$

since the formulas $\varphi_{Y /(Y / C), A}$ yield a partition of $Y / C$, and since

$$
|C| \chi_{(c)}\left(\left[\varphi_{Y /(Y / C), A}\right]\right)=|A| \chi_{(c)}\left(\left[\varphi_{Y /(Y / A), A}\right]\right)
$$

by property (ii). This recursion formula determines $\chi_{(c)}\left(\left[\varphi_{Y /(Y / C), C}\right]\right)$.

2. Existence: By Lemma 8.6 and Lemma 8.4 the map $\chi_{(c)}$ from definition 8.1 factors to a ring morphism

$$
\chi_{(c)}: K_{0}\left(P F F_{S}\right) \rightarrow K_{0}^{m o t}\left(\operatorname{Var}_{S}\right) \otimes \mathbb{Q}
$$

The fact that property (i) is satisfied, follows immediately from the definition. Let us prove property (ii). Put $G=G(Y / X)$, and denote by $\psi: C \rightarrow G$ the inclusion. We have $Y_{C}=\operatorname{Res}_{\psi} Y_{G}$, where $Y_{C}$ is the variety $Y$ with good $C$-action as a Galois cover of $Y / C$, and $Y_{G}$ is the variety $Y$ with good $G$-action as a Galois cover of $X$. If $\operatorname{Con}(Y / X)$ is the conjugation class of $C$ in $G$, and if we denote by $\operatorname{Con}(Y /(Y / C))$ the conjugation domain $\{C\}$, then

$$
\alpha_{C o n(Y / X)}=\frac{|C|}{\left|N_{G}(C)\right|} \operatorname{Ind}_{\psi} \alpha_{C o n(Y /(Y / C))}
$$

Hence, we may conclude by Lemma 7.20

Corollary 8.8. There exists a canonical ring morphism

$$
K_{0}\left(P F F_{S}\right) \rightarrow K_{0}\left(D^{b}\left(S, \mathbb{Q}_{\ell}\right)\right) \otimes \mathbb{Q}
$$

mapping a ring formula $\varphi$ defining a locally closed subset $X$ of $\mathbb{A}_{S}^{m}$, to the class of $R \pi_{!}\left(\mathbb{Q}_{\ell}\right)$, where $\pi: \mathbb{A}_{S}^{m} \rightarrow S$ is the structural morphism.

Proof. The morphism is obtained by composing $\chi_{(c)}$ with the realization morphism from Corollary 6.16 By point (i) of Theorem 8.7 the image of $[\varphi]$ is nothing but the image of $\chi_{(c)}(X)$ in $K_{0}\left(D^{b}\left(S, \mathbb{Q}_{\ell}\right)\right)$. Let us show that this image coincides with the class of $R \pi_{!}\left(\mathbb{Q}_{\ell}\right)$. By additivity of $R \pi_{!}$, and constructible resolution of singularities, 
we may suppose that $X$ is smooth and proper over $S$. In this case, the result follows simply by definition of the realization functor $\mathcal{M}_{+}^{o}(S) \rightarrow D^{b}\left(S, \mathbb{Q}_{\ell}\right)$.

Proposition 8.9 (Base Change). Let $T$ be another $k$-variety, with a morphism $f$ : $T \rightarrow S$. This morphism induces base change morphisms $K_{0}\left(P F F_{S}\right) \rightarrow K_{0}\left(P F F_{T}\right)$ and $K_{0}^{m o t}\left(\operatorname{Var}_{S}\right) \otimes \mathbb{Q} \rightarrow K_{0}^{m o t}\left(\operatorname{Var}_{T}\right) \otimes \mathbb{Q}$, which we both denote by $f^{*}$. We get a commutative diagram

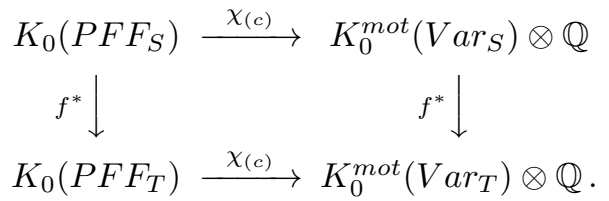

Proof. Let $\varphi\left(x_{1}, \ldots, x_{m}\right)$ be a ring formula over $S$, and let $\varphi^{\prime}$ be its pullback to $T$. Suppose that $\varphi$ is equivalent to a quantifier-free Galois formula $\theta$, with corresponding Galois stratification $\mathcal{A}=<A_{i}, C_{i} / A_{i}, \operatorname{Con}\left(A_{i}\right)>$, i.e. $Z(\varphi, x, M)=Z(\mathcal{A}, x, M)$ for each point $x$ of $S$, and each pseudo-finite field extension $M$ of the residue field $k(x)$. Let $\theta^{\prime}$ be the Galois formula, associated to a Galois stratification of $\mathbb{A}_{T}^{m}$, obtained by pulling back $\mathcal{A}$ via the morphism $\mathbb{A}_{T}^{m} \rightarrow \mathbb{A}_{S}^{m}$ induced by $f$, as explained in Section 3.5. We denote this Galois stratification by $\mathcal{A}^{\prime}=<A_{j}^{\prime}, C_{j}^{\prime} / A_{j}^{\prime}, \operatorname{Con}\left(A_{j}^{\prime}\right)>$. It is clear that $\varphi^{\prime}$ is equivalent to $\theta^{\prime}$.

Now we compare $\chi_{(c)}(\theta)$ and $\chi_{(c)}\left(\theta^{\prime}\right)$. We may suppose that the support of $\mathcal{A}$ consists of a single stratum $A$, with Galois cover $C / A$ and conjugation domain $\operatorname{Con}(A)$. By a stratification argument, we may assume that $T$ is irreducible and smooth, and that $A^{\prime}:=A \times_{k} T$ is a stratum of $\mathcal{A}^{\prime}$. It suffices to prove that

$$
f^{*} \chi_{c, \alpha_{\text {Con }(A)}}([C])=\chi_{c, \alpha_{\text {Con }\left(A^{\prime}\right)}}\left(\left[C^{\prime}\right]\right)
$$

where $C^{\prime} / A^{\prime}$ and $C o n\left(A^{\prime}\right)$ are the induced Galois cover and conjugation domain; i.e. $C^{\prime}$ is a connected component of $C \times{ }_{A} A^{\prime}$, and $\operatorname{Con}\left(A^{\prime}\right)$ is the set of elements of $\operatorname{Con}(A)$ which are contained in $G\left(C^{\prime} / A^{\prime}\right)$. We denote by $\psi: G\left(C^{\prime} / A^{\prime}\right) \rightarrow G(C / A)$ the inclusion. By Lemma 7.24 .

$$
f^{*} \chi_{c, \alpha_{C o n(A)}}([C])=\chi_{c, \alpha_{C o n(A)}}\left(\left[C \times{ }_{S} T\right]\right)
$$

while $C \times{ }_{S} T=\operatorname{Ind} d_{\psi} C^{\prime}$ and $\alpha_{C o n\left(A^{\prime}\right)}=\operatorname{Res}_{\psi} \alpha_{\operatorname{Con}(A)}$. So we can conclude by applying Lemma 7.18

\section{The Relative motivic Poincaré Series}

Let $S$ be a variety over a field $k$ of characteristic zero. Let $X$ be a separated scheme, of finite type over $S$. For any integer $n>0$, we denote by $S[t] /\left(t^{n}\right)$ the $S$-scheme $S \times{ }_{k} \operatorname{Spec} k[t] /\left(t^{n}\right)$. We define, for each integer $n \geq 0$, a contravariant functor

$$
F_{n}:\left(S c h_{S}\right)^{o p} \rightarrow(\text { Sets })
$$

from the category $\left(S c h_{S}\right)$ of $S$-schemes, to the category of sets, by

$$
F_{n}(Y)=\operatorname{Hom}_{S c h_{S}}\left(Y \times_{S} S[t] /\left(t^{n+1}\right), X\right)
$$

For any field $K$ containing $k$, we call the points of $F_{n}(\operatorname{Spec} K)$ the $K$-valued relative $n$-jets on $X / S$.

Proposition 9.1. The functor $F_{n}$ is representable by a separated $S$-scheme of finite type $\mathcal{L}_{n}(X / S)$, for each $n$. 
Proof. The proof is analogous to the proof in the absolute case $S=\operatorname{Spec} k$. Suppose that $X$ is affine over $S$, with $X=\operatorname{Spec} \mathcal{O}_{S}\left[x_{1}, \ldots, x_{m}\right] /\left(f_{1}, \ldots, f_{r}\right)$. Let

$$
a=\left(a_{1,0}+a_{1,1} t+\ldots+a_{1, n} t^{n}, \ldots, a_{m, 0}+\ldots, a_{m, n} t^{n}\right)
$$

be an $m$-tuple of elements of $\mathcal{O}_{S}[t] /\left(t^{n+1}\right)$. The system of equations $f_{i}(a) \equiv 0$ $\bmod t^{n+1}$, for $i=1, \ldots, r$, puts algebraic conditions on the coefficients $a_{i, j}$, and if we consider these coefficients as affine coordinates on $\mathbb{A}_{S}^{m(n+1)}$, the $f_{i}$ define a closed subscheme $\mathcal{L}_{n}(X / S)$ of $\mathbb{A}_{S}^{m(n+1)}$. A gluing procedure yields the general case.

Note that $\mathcal{L}_{0}(X / S) \cong X$. By Yoneda's Lemma, the truncation map $S[t] /\left(t^{n+1}\right) \rightarrow$ $S[t] /\left(t^{n}\right)$ induces a truncation morphism of $S$-schemes

$$
\pi_{n}^{n+1}: \mathcal{L}_{n+1}(X / S) \rightarrow \mathcal{L}_{n}(X / S)
$$

for each $n \geq 0$. These morphisms are affine, and hence, we can take the projective limit in the category of $S$-schemes to obtain an $S$-scheme $\mathcal{L}(X / S)$. It comes with natural projections $\pi_{n}: \mathcal{L}(X / S) \rightarrow \mathcal{L}_{n}(X / S)$. For any field $K$ containing $k$, we call the points of $\mathcal{L}(X / S)(K)$ the $K$-valued relative arcs on $X / S$.

When $X$ is smooth over $S$, the morphisms $\pi_{n}^{n+1}$ are piecewisely trivial fibrations with fiber $\mathbb{A}_{S}^{d}$, where $d$ is the relative dimension of $X$. An $S$-morphism $h$ from $X$ to $X^{\prime}$ induces a morphism $h$ from $\mathcal{L}(X / S)$ to $\mathcal{L}\left(X^{\prime} / S\right)$ by composition, and a morphism of $k$-varieties $f: W \rightarrow S$ induces a pull-back morphism $f^{*}: \mathcal{L}(X / S) \rightarrow$ $\mathcal{L}\left(X \times{ }_{S} W / W\right)$. If $Y$ is a separated $W$-scheme of finite type, this morphism $f$ induces also a forgetful morphism $f_{*}: \mathcal{L}(Y / W) \rightarrow \mathcal{L}(Y / S)$.

Lemma 9.2. For any $k$-variety $T$, endowed with a morphism $f: T \rightarrow S$, and for any separated $S$-scheme $X$ of finite type, there are canonical isomorphisms of T-schemes

$$
\begin{aligned}
\mathcal{L}_{n}\left(X \times_{S} T / T\right) & \cong \mathcal{L}_{n}(X / S) \times_{S} T, \\
\mathcal{L}\left(X \times_{S} T / T\right) & \cong \mathcal{L}(X / S) \times_{S} T,
\end{aligned}
$$

compatible with the truncation morphisms $\pi_{n}^{m}$ and $\pi_{n}$.

Proof. Denote, for any variety $U$, the category of $U$-schemes by $S c h_{U}$. The scheme $\mathcal{L}_{n}(X / S) \times{ }_{S} T$ represent the functor

$$
G_{n}:\left(S c h_{T}\right)^{o p} \rightarrow(\text { Sets })
$$

mapping a $T$-scheme $Y$ to

$$
\begin{aligned}
\operatorname{Hom}_{S c h_{T}}\left(Y, \mathcal{L}_{n}(X / S) \times_{S} T\right) & =\operatorname{Hom}_{S c h_{S}}\left(Y, \mathcal{L}_{n}(X / S)\right) \\
& =\operatorname{Hom}_{S c c_{S}}\left(Y \times_{S} S[t] /\left(t^{n+1}\right), X\right) .
\end{aligned}
$$

There is a canonical isomorphism of $T$-schemes

$$
Y \times_{S} S[t] /\left(t^{n+1}\right) \cong Y \times_{T} T[t] /\left(t^{n+1}\right)
$$

and we have

$$
\operatorname{Hom}_{S c h_{S}}\left(Y \times_{S} S[t] /\left(t^{n+1}\right), X\right)=\operatorname{Hom}_{S c h_{T}}\left(Y \times_{T} T[t] /\left(t^{n+1}\right), X \times_{S} T\right)
$$

Hence, there is a natural equivalence of functors between $G_{n}$ and the functor represented by $\mathcal{L}_{n}\left(X \times_{S} T / T\right)$, and, by Yoneda's lemma, it corresponds to an isomorphism

$$
\mathcal{L}_{n}\left(X \times{ }_{S} T / T\right) \cong \mathcal{L}_{n}(X / S) \times{ }_{S} T
$$

Taking projective limits over $n$ concludes the proof. 
For any scheme $Y$, we denote by $Y_{\text {red }}$ the underlying reduced scheme.

Lemma 9.3. For any separated $S$-scheme $X$ of finite type, there exists an integer $a>0$, such that, for each $n \geq 0$, the truncation morphism

$$
\mathcal{L}_{n a}(X / S)_{\text {red }} \rightarrow \mathcal{L}_{n}(X / S)
$$

factors through a morphism

$$
\mathcal{L}_{n a}(X / S)_{\text {red }} \rightarrow \mathcal{L}_{n}\left(X_{\text {red }} / S\right)
$$

Proof. We may assume that $X$ and $Y:=\mathcal{L}_{n a}(X / S)_{\text {red }}$ are affine, say $X=\operatorname{Spec} B$, and $Y=\operatorname{Spec} A$. Denote by $\mathcal{N}_{B}$ the nilradical of $B$. We have to prove that there exists an integer $a>0$, such that, for each $n \geq 0$, for each reduced $k$-algebra $A$, and for each morphism of $k$-algebras

$$
g: B \rightarrow A[t] /\left(t^{n a+1}\right)
$$

the composition $h: B \rightarrow A[t] /\left(t^{n+1}\right)$ factors through $B / \mathcal{N}_{B}$.

In other words, we have to show that the ideal $g^{-1}\left(t^{n+1}\right)$ contains $\mathcal{N}_{B}$. Since $B$ is Noetherian, we can choose a positive integer $a$, such that $b^{a}=0$ for any element $b$ of $\mathcal{N}_{B}$. Since $A$ is reduced, $g(b)$ has to belong to the ideal $\left(t^{n+1}\right)$, as soon as $b \in \mathcal{N}_{B}$.

For any point $x$ on $S$ and any separated $S$-scheme of finite type $X$, the fiber of $\mathcal{L}(X / S)$ over $x$ is canonically isomorphic to $\mathcal{L}\left(X \times_{S} x\right)$, where $\mathcal{L}($.$) denotes the arc$ scheme as defined in [12, p.1. Hence, for any field $K$, giving a $K$-valued relative $\operatorname{arc} \psi$ on $X / S$, amounts to giving an arc in $\left(X \times_{x} S\right)(K[[t]])$, where $x$ denotes the image of $\pi_{0}(\psi)$ in $S$.

For any separated $S$-scheme $Y$ of finite type, we put $[Y]:=\left[Y_{\text {red }}\right]$ in $K_{0}\left(\operatorname{Var}_{S}\right)$.

Definition 9.4. If $X$ is a separated $S$-scheme of finite type, we define its relative Igusa Poincaré series as

$$
Q(X / S ; T)=\sum_{n=0}^{\infty}\left[\mathcal{L}_{n}(X / S)\right] T^{n} \quad \in K_{0}\left(\operatorname{Var}_{S}\right)[[T]]
$$

We can also generalize Denef and Loeser's definition of the geometric and arithmetic Poincaré series [11, 13, 14, 15, 16] to our relative setting. The aim is to define $P_{\text {geom }}(X / S ; T)$ and $P_{\text {arith }}(X / S ; T)$ as objects in $K_{0}\left(\operatorname{Var}_{S}\right)[[T]]$, resp.

$\left(K_{0}^{m o t}\left(\operatorname{Var}_{S}\right) \otimes \mathbb{Q}\right)[[T]]$, such that base-change to any point $x$ of $S$, yields the "classical" motivic Poincaré series of the fiber $X \times_{S} x$.

First, we need to prove a uniform version of Greenberg's Theorem 23]. We will show that there exist positive integers $c, e$, such that, for any point $x$ on $S$, for any positive integer $n$, and for any field extension $K$ of $k(x)$, the projections $\pi_{n} \mathcal{L}\left(X_{x}\right)(K)$ and $\pi_{n}^{c n+e} \mathcal{L}_{c n+e}\left(X_{x}\right)(K)$ coincide, where $X_{x}$ denotes the fiber $X \times_{S} x$ of $X$ over $x$. In other words, a $K$-valued $n$-jet on a fiber $X_{x}$ lifts to a $K$-valued arc on $X_{x}$, as soon as it lifts to a $K$-valued $(c n+e)$-jet on $X_{x}$. Using our relative arc and jet spaces, we can write this property as

$$
\pi_{n}(\mathcal{L}(X / S)(K))=\pi_{n}^{c n+e}\left(\mathcal{L}_{c n+e}(X / S)(K)\right)
$$

We will proceed in two steps. We start by giving a short alternative proof of the absolute case, using resolution of singularities. Then, we show how to extend this proof to obtain the uniform version stated above. First of all, we recall the following definition. 
Definition 9.5. Let $X$ be a separated scheme of finite type over $k$, and let $\mathcal{I}$ be an ideal sheaf on $X$. For any field $K$ containing $x$, and any arc $\psi$ in $\mathcal{L}(X)(K)$, we put $x:=\pi_{0}(\psi) \in X$, and we define the order of $\mathcal{I}$ at $\psi$ by

$$
\operatorname{ord}_{\mathcal{I}}(\psi):=\min \left\{\operatorname{ord}_{t} f(\psi) \mid f \in \mathcal{I}_{x}\right\} \in \mathbb{N} \cup\{\infty\}
$$

If $Z$ is a closed subscheme of $X$, we denote the defining ideal sheaf of $Z$ in $X$ by $\mathcal{I}_{Z}$, and we put

$$
\operatorname{ord}_{Z}(\psi):=\operatorname{ord}_{\mathcal{I}_{Z}}(\psi)
$$

We call this value the contact order of $\psi$ with $Z$. Note that $\operatorname{ord}_{Z}(\psi)=\infty$ iff $\psi$ is contained in $Z$.

Theorem 9.6 (Greenberg). Let $Z$ be a separated scheme of finite type over $k$. There exist positive integers $c, e$, such that, for any positive integer $n$, and for any field extension $K$ of $k$, the projections $\pi_{n} \mathcal{L}(Z)(K)$ and $\pi_{n}^{c n+e} \mathcal{L}_{c n+e}(Z)(K)$ coincide.

Proof. We embed $Z$ as a subscheme in some smooth ambient $k$-variety $Y$, and we take an embedded principalization $h: Y^{\prime} \rightarrow Y$ for the defining ideal sheaf $\mathcal{I}_{Z}$ of $Z$ in the structure sheaf $\mathcal{O}_{Y}$, defined over $k$, such that $h$ is an isomorphism over the complement $Y \backslash Z$, see [6, 2.5]. This means that $h$ is a proper birational morphism from a smooth variety $Y^{\prime}$ to $Y$, such that

$$
\mathcal{I}_{Z} \cdot \mathcal{O}_{Y^{\prime}}=\mathcal{O}_{Y^{\prime}}\left(-\sum_{i=1}^{N} r_{i} E_{i}\right)
$$

where $E=\sum_{i=1}^{N} E_{i}$ is a normal crossing divisor on $Y^{\prime}$, and where the $r_{i}$ are positive integers. We denote the maximum of the multiplicities $r_{i}$ by $R$.

Choose an integer $n>0$. Let $K$ be a field extension of $k$, and let $\psi$ be a $K$-valued arc on $Y$, such that

$$
n N R \leq \operatorname{ord}_{Z}(\psi)<\infty
$$

This means that

$$
\pi_{n N R-1}(\psi): \operatorname{Spec} K[t] /\left(t^{n N R}\right) \rightarrow Y
$$

factors through a $(n N R-1)$-jet on $Z$. Since $h$ is an isomorphism over $Y \backslash Z$, it follows from the valuative criteria for properness and separateness, that there exists a unique $K$-valued $\operatorname{arc} \psi^{\prime}$ on $Y^{\prime}$, such that $h \circ \psi^{\prime}=\psi$. We put $\nu_{i}=\operatorname{ord}_{E_{i}}\left(\psi^{\prime}\right)$, and we denote by $\nu$ the maximum of the $\nu_{i}$. We may assume that $\nu=\nu_{1}$.

Now, observe that $n N R \leq \operatorname{ord}_{Z}(\psi)=\sum_{i=1}^{N} r_{i} \nu_{i} \leq \nu N R$, hence $\nu \geq n$. Since the divisor $E_{1}$ is smooth, there exists an arc $\zeta^{\prime}$ in $\mathcal{L}\left(E_{1}\right)(K)$ such that $\pi_{\nu-1}\left(\zeta^{\prime}\right)=$ $\pi_{\nu-1}\left(\psi^{\prime}\right)$ in $\mathcal{L}_{\nu_{1}}\left(E_{1}\right)(K)$. If we denote by $\zeta$ the image of $\zeta^{\prime}$ under $h$, then $\pi_{\nu-1}(\zeta)=$ $\pi_{\nu-1}(\psi)=h\left(\pi_{\nu-1}\left(\psi^{\prime}\right)\right)$ in $\mathcal{L}_{\nu-1}(Z)$, and, a fortiori, $\pi_{n-1}(\zeta)=\pi_{n-1}(\psi)$.

Hence, we see that, for any $K$-valued $n$-jet $\psi_{n}$ on $Z$, this jet $\psi_{n}$ lifts to a $K$-valued arc on $Z$, iff it lifts to a $K$-valued $(n N R+N R-1)$-jet on $Z$.

Theorem 9.7 (Uniform version). Let $S$ be a variety over $k$, and let $X$ be a separated scheme of finite type over $S$. There exist positive integers $c, e$, such that, for any point $x$ on $S$, for any positive integer $n$, and for any field extension $K$ of $k(x)$, the projections $\pi_{n} \mathcal{L}\left(X_{x}\right)(K)$ and $\pi_{n}^{c n+e} \mathcal{L}_{c n+e}\left(X_{x}\right)(K)$ coincide, where $X_{x}$ denotes the fiber of $X$ over $x$. 
Proof. By the proof of the previous theorem, it suffices to find, for each fiber $X_{x}$, an embedding in some smooth ambient space, and an embedded principalization for its defining ideal sheaf, such that the numbers $N, R$ are uniformly bounded. This follows from the existence of a constructible embedded resolution, by Proposition 5.4

Definition 9.8. If $X$ is a separated $S$-scheme of finite type, we define its relative geometric Poincaré series as

$$
P_{\text {geom }}(X / S ; T)=\sum_{n=0}^{\infty}\left[\pi_{n} \mathcal{L}(X / S)\right] T^{n} \quad \in K_{0}\left(\operatorname{Var}_{S}\right)[[T]]
$$

By Theorem 9.7 $\left(\pi_{n} \mathcal{L}(X / S)\right)_{\text {red }}=\left(\pi_{n}^{n^{\prime}} \mathcal{L}_{n^{\prime}}(X / S)\right)_{\text {red }}$ for some integer $n^{\prime}>n$, hence the truncation $\left(\pi_{n} \mathcal{L}(X / S)\right)_{\text {red }}$ is, by Chevalley's Theorem, a constructible subset of $\mathcal{L}_{n}(X / S)_{\text {red }}$, and its Grothendieck bracket is well-defined.

Proposition 9.9 (Base Change). Let $X$ be an $S$-variety, and let $R(X / S ; T)$ be either its relative Igusa Poincaré series, or its relative geometric Poincaré series. Let $W \rightarrow S$ be a morphism of $k$-varieties. If we take the image of the coefficients of $R$ under the base change morphism $K_{0}\left(\operatorname{Var}_{S}\right) \rightarrow K_{0}\left(V a r_{W}\right)$, then we obtain the corresponding Poincaré series $R\left(X \times_{S} W ; T\right)$.

Proof. This follows immediately from Lemma 9.2

In particular, by base change to any point $x$ of $S$, we recover the absolute Poincaré series of the (not necessarily reduced) fiber $X_{x}$.

Now, we define the relative arithmetic Poincaré series. If $X$ is affine over $S$, say $X$ is a closed subscheme of $\mathbb{A}_{S}^{m}$, Theorem 9.7 implies that we can find, for each positive integer $n$, a ring formula $\psi_{n}\left(x_{1}, \ldots, x_{(n+1) m}\right)$ over $S$, such that, for any point $x$ on $S$, and any field extension $K$ of $k(x)$, the $(n+1) m$-tuples over $K$ satisfying $\psi_{n}$, correspond to the points of $\pi_{n}\left(\mathcal{L}\left(X_{x}\right)(K)\right)$. Here we identified $\pi_{n}\left(\mathcal{L}\left(X_{x}\right)(K)\right)$ with a subset of $\mathcal{L}_{n}\left(\mathbb{A}_{k(x)}^{m}\right)(K)=\mathbb{A}_{k(x)}^{(n+1) m}(K)$.

Definition 9.10. We define the relative arithmetic Poincaré series as

$$
P_{\text {arith }}(X / S ; T)=\sum_{n=0}^{\infty} \chi_{(c)}\left(\left[\psi_{n}\right]\right) T^{n} \quad \in\left(K_{0}^{m o t}\left(\operatorname{Var}_{S}\right) \otimes \mathbb{Q}\right)[[T]]
$$

If $X$ is not affine over $S$, we can still define its arithmetic Poincaré series, using definable subassignements as in [15].

Proposition 9.11 (Base Change). Let $X$ be a separated $S$-scheme of finite type, and let $W \rightarrow S$ be a morphism of $k$-varieties. If we take the image of the coefficients of $P_{\text {arith }}(X / S ; T)$ under the base change morphism $K_{0}^{m o t}\left(\operatorname{Var}_{S}\right) \otimes \mathbb{Q} \rightarrow$ $K_{0}^{m o t}\left(\operatorname{Var}_{W}\right) \otimes \mathbb{Q}$, then we obtain the arithmetic Poincaré series $P_{\text {arith }}\left(X \times_{S} W ; T\right)$.

Proof. This follows from Lemma 8.9

\section{ACKNOWLEDGEMENTS}

The author would like to thank François Loeser for proposing the subject of this article. 


\section{REFERENCES}

[1] Revêtements étales et groupe fondamental. Springer-Verlag, Berlin, 1971. Séminaire de Géométrie Algébrique du Bois Marie 1960-1961 (SGA 1), Dirigé par Alexandre Grothendieck. Augmenté de deux exposés de M. Raynaud, Lecture Notes in Mathematics, Vol. 224.

[2] D. Abramovich, K. Karu, K. Matsuki, and J. Włodarczyk. Torification and factorization of birational maps. J. Am. Math. Soc., 15(3):531-572, 2002, arxiv:math.AG/9904135.

[3] J. Ax. The elementary theory of finite fields. Ann. of Math., 88:239-271, 1968.

[4] A. Beauville. Varits de Prym et jacobiennes intermediaires. Ann. Sci. Éc. Norm. Supér., 10:309-391, 1977.

[5] F. Bittner. Euler characteristics of Varieties and Mappings. PhD thesis, Utrecht, 2003.

[6] A. Bravo, S. Encinas, and O. Villamayor. A simplified proof of desingularization and applications. Rev. Mat. Iberoamericana, 21(2):349-458, 2005.

[7] R. Cluckers and F. Loeser. Constructible motivic functions, integrals with parameters, and cell decomposition. arxiv:math.AG/0410203.

[8] R. Cluckers and F. Loeser. Fonctions constructibles et intégration motivique i. C. R. Ac. Sci., 339:411-416, 2004.

[9] R. Cluckers and F. Loeser. Fonctions constructibles et intégration motivique ii. C. R. Ac. Sci., 339:487-492, 2004.

[10] S. del Baño Rollin and V. Navarro Aznar. On the motive of a quotient variety. Collect. Math, 49:203-226, 1998.

[11] J. Denef and F. Loeser. Motivic Igusa zeta functions. J. Algebraic Geom., 7:505-537, 1998, arxiv:math.AG/9803040.

[12] J. Denef and F. Loeser. Germs of arcs on singular algebraic varieties and motivic integration. Invent. Math., 135:201-232, 1999, arxiv:math.AG/9803039.

[13] J. Denef and F. Loeser. Definable sets, motives and p-adic integrals. J. Am. Math. Soc., 14(2):429-469, 2001, arxiv:math.AG/9910107.

[14] J. Denef and F. Loeser. Geometry on arc spaces of algebraic varieties. Progr. Math., 201:327348, 2001, arxiv:math.AG/0006050.

[15] J. Denef and F. Loeser. Motivic integration and the Grothendieck group of pseudo-finite fields. Proceedings of the International Congress of Mathematicians (ICM 2002), 2:13-23, 2002, arxiv:math.AG/0207163.

[16] J. Denef and F. Loeser. On some rational generating series occuring in arithmetic geometry. In Geometric aspects of Dwork theory I, pages 509-526. de Gruyter, 2004, arxiv:math. NT/0212202.

[17] C. Deninger and J. Murre. Motivic decomposition of abelian schemes and the fourier transform. J. reine Angew. Math., 422:201-219, 1991.

[18] T. Ekedahl. On the adic formalism. In The Grothendieck Festschrift, Vol. II, volume 87 of Progr. Math., pages 197-218. Birkhäuser Boston, 1990.

[19] M. Fried and M. Jarden. Field Arithmetic. Springer-Verlag, 1986.

[20] M. Fried and G. Sacerdote. Solving Diophantine problems over all residue class fields of a number field and all finite fields. Ann. of Math., 104:203-233, 1976.

[21] W. Fulton. Intersection theory, volume 2 of Ergebnisse der Math. und ihrer Grenzgebiete. Springer Verlag, 1984.

[22] H. Gillet and C. Soulé. Descent, motives and K-theory. J. Reine Angew. Math., 478:127-176, 1996, arxiv:math.AG/9507013.

[23] M. Greenberg. Rational points in Henselian discrete valuation rings. Publ. Math. Inst. Hautes Étud. Sci., 31:59-64, 1966.

[24] M. J. Greenberg and J. R. Harper. Algebraic topology, volume 58 of Mathematics Lecture Note Series. Benjamin/Cummings Publishing Co. Inc. Advanced Book Program, Reading, Mass., 1981. A first course.

[25] Ph. Griffiths and J. Harris. Principles of algebraic geometry. Wiley-Interscience [John Wiley \& Sons], New York, 1978. Pure and Applied Mathematics.

[26] A. Grothendieck. Sur quelques propriétés fondamentales en théorie des intersections. Sminaire Claude Chevalley, 3(4):36 p., 1958.

[27] F. Guillen and V. Navarro-Aznar. Un critère d'extension d'un foncteur défini sur les schémas lisses. Publ. Math., Inst. Hautes Étud. Sci., (95):1-91, 2002. 
[28] H. Hironaka. Resolution of singularities of an algebraic variety over a field of characteristic zero. I, II. Ann. of Math., 79(2):109-326, 1964.

[29] M. Levine. Mixed motives, volume 57 of Mathematical surveys and monographs. AMS, 1998, http://www.ams.org/online_bks/surv57.

[30] Q. Liu. Algebraic geometry and arithmetic curves, volume 6 of Oxford Graduate Texts in Mathematics. Oxford University Press, 2002.

[31] Yu. Manin. Correspondences, motifs and monoidal transformations. Mat. Sb., N. Ser., 77(119):475-507, 1968.

[32] D. Marker. Model theory: An introduction. Graduate Texts in Mathematics. 217. New York, NY: Springer. viii, 342 p., 2002.

[33] J. P. Murre. Lectures on an Introduction to Grothendieck's Theory of the Fundamental Group. Tata Institute of Fundamental Research, 1967.

[34] A. J. Scholl. Classical motives. In Motives, Seattle, 1991, volume 55, pages 163-187. Amer. Math. Soc., 1994.

[35] J.-P. Serre. Zeta and L functions. In Arithmetic Algebraic Geometry, Harper's Series in Modern Math., pages 82-92, 1965.

[36] O. Villamayor. Patching local uniformizations. Ann. Sci. Éc. Norm. Supér., 25(6):629-677, 1992.

Université Lille 1, Laboratoire Painlevé, CNRS - UMR 8524, Cité Scientifique, 59 655 Villeneuve D'AscQ Cédex, France

E-mail address: johannes.nicaise@math.univ-lille1.fr 